\title{
Horizontal Convective Boiling of R448A, R449A, and R452B within a Micro-Fin Tube with Extensive Measurement and Analysis Details
}

\author{
Mark A. Kedzierski \\ Donggyu Kang
}

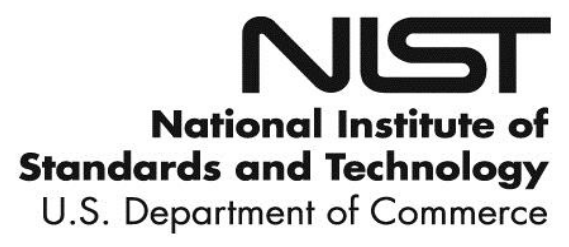




\title{
Horizontal Convective Boiling of R448A, $\mathrm{R} 449 \mathrm{~A}$, and $\mathrm{R} 452 \mathrm{~B}$ within a Micro-Fin Tube with Extensive Measurement and Analysis Details
}

\author{
Mark A. Kedzierski \\ Donggyu Kang \\ Energy and Environment Division \\ Engineering Laboratory
}

This publication is available free of charge from: http://dx.doi.org/10.6028/NIST.TN.1915

April 2016

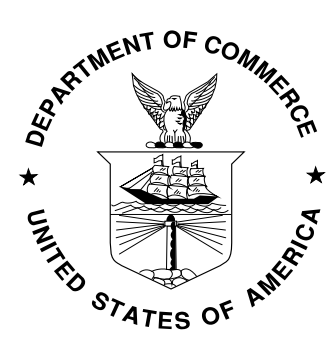

U.S. Department of Commerce

Penny Pritzker, Secretary

National Institute of Standards and Technology 
Certain commercial entities, equipment, or materials may be identified in this document in order to describe an experimental procedure or concept adequately. Such identification is not intended to imply recommendation or endorsement by the National Institute of Standards and Technology, nor is it intended to imply that the entities, materials, or equipment are necessarily the best available for the purpose.

National Institute of Standards and Technology Technical Note 1915

Natl. Inst. Stand. Technol. Tech. Note 1915, 72 pages (April 2016) http://dx.doi.org/10.6028/NIST.TN.1915 CODEN: NTNOEF 


\title{
Horizontal Convective Boiling of R448A, R449A, and R452B within a Micro-Fin Tube with Extensive Measurement and Analysis Details
}

\author{
M. A. Kedzierski and D-G. Kang \\ National Institute of Standards and Technology \\ Gaithersburg, MD 20899
}

\begin{abstract}
This report presents local convective boiling measurements in a micro-fin tube for three low global warming potential (GWP) refrigerants: R448A, R449A, and R452B ${ }^{1}$, which are possible replacements for R404A. Water heating either in counterflow or in parallel flow with the test refrigerant was used to vary the heat flux for a given quality. An existing correlation from the literature was modified to predict multicomponent mixtures, which predicted $98 \%$ of the measurements to within $\pm 20 \%$. The new correlation was used to compare the heat transfer coefficient of the three test fluids at the same heat flux, saturated refrigerant temperature, and refrigerant mass flux. The resulting comparison showed that refrigerant R452B exhibited the highest heat transfer performance, in large part due to its approximate $28 \%$ larger liquid thermal conductivity and smaller temperature glide as compared to the tested low-GWP refrigerants. For the example case, the heat transfer coefficient for R449A was approximately $8 \%$ larger than that for R448A, while the heat transfer coefficient for R452B was more than $59 \%$ larger than either R448A or R449A. As predicted by the modified model, the heat transfer coefficients for R448A and R449A are roughly between $26 \%$ and $48 \%$ less than that of R404A for most qualities between $10 \%$ and $70 \%$. The smaller heat transfer coefficient of R448A and R449A as compared to that of R404A results even though these refrigerants both have an approximate $20 \%$ larger liquid thermal conductivity than R404A. In contrast, the model predicts that the R452B heat transfer coefficient is approximately $13 \%$ larger than that of R404A for thermodynamic qualities between $10 \%$ and $70 \%$. The measurements are important as part of the evaluation of low-GWP replacement refrigerants for R410A and R404A for the air-conditioning and refrigeration markets, respectively.
\end{abstract}

Keywords: $\quad$ boiling, enhanced heat transfer, low-GWP, micro-fin, refrigerant mixtures

\footnotetext{
${ }^{1} \mathrm{R} 452 \mathrm{~B}$ is the pending ASHRAE designation for the mixture R32/ R125/R1234yf (67/7/26), which has the developmental designation DR-55.
} 


\section{TABLE OF CONTENTS}

ABSTRACT

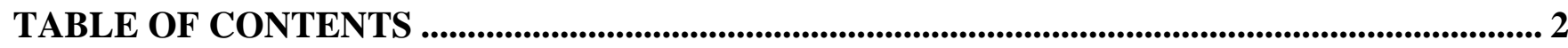

LIST OF TABLES ......................................................................................................................................... 3

INTRODUCTION............................................................................................................................................. 4

EXPERIMENTAL APPARATUS............................................................................................................... 5

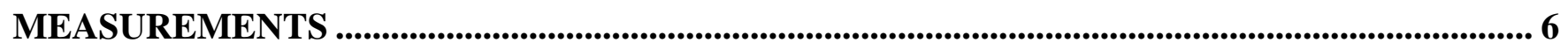

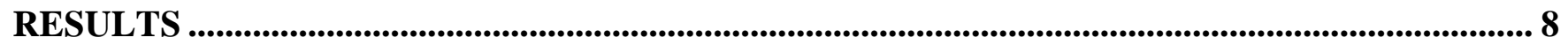

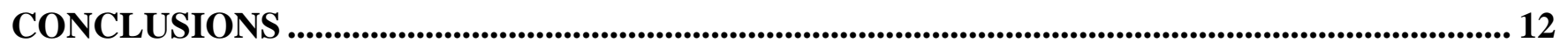

ACKNOWLEDGEMENTS ................................................................................................................................... 13

NOMENCLATURE............................................................................................................................................ 14

APPENDIX A1 ............................................................................................................................................................ 34

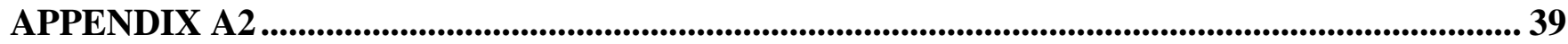

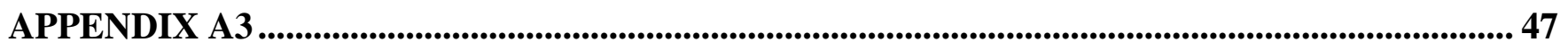

APPENDIX B1 .......................................................................................................................................................... 53

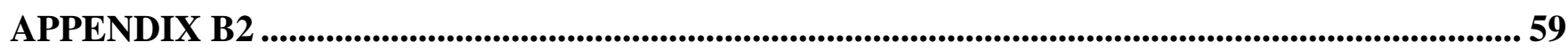

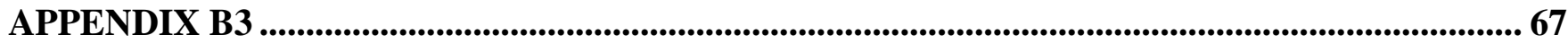




\section{LIST OF TABLES}

Table 1 Median estimated $95 \%$ relative expanded uncertainties for measurements (U)

\section{LIST OF FIGURES}

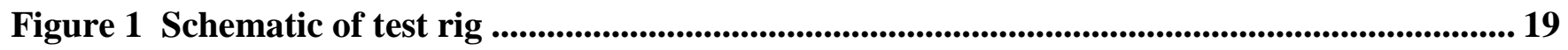

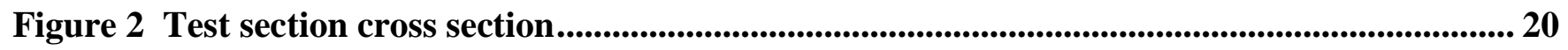

Figure 3 Detailed schematic of test section ...................................................................................... 21

Figure 4 Relative uncertainty of inner wall temperature........................................................ 22

Figure 5 Counterflow temperature profiles for a 449A test ............................................................... 23

Figure 6 Relative uncertainty of water temperature gradient with respect to quality .................. 24

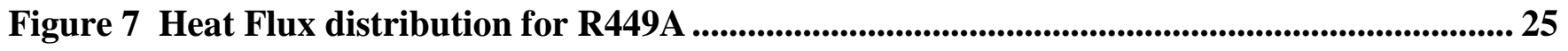

Figure 8 Relative uncertainty of the Nusselt number with respect to the quality ......................... 26

Figure 9 Comparison between measured Nusselt numbers and those predicted by the modified

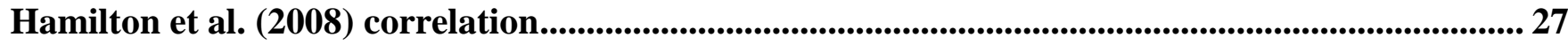

Figure 10 Flow boiling heat transfer coefficient for micro-fin tube versus thermodynamic quality for R452B, R449A, and R448A (counterflow) .............................................................................28

Figure 11 Flow boiling heat transfer coefficient for micro-fin tube versus thermodynamic quality for R452B, R449A, and R448A (parallel flow) ....................................................................................29

Figure 12 Flow boiling heat transfer coefficient versus thermodynamic quality for test

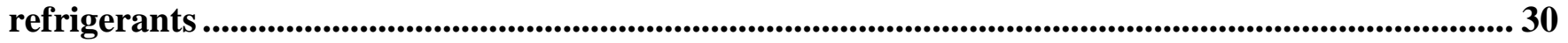

Figure 13 Flow boiling heat transfer coefficient versus thermodynamic quality for R404A

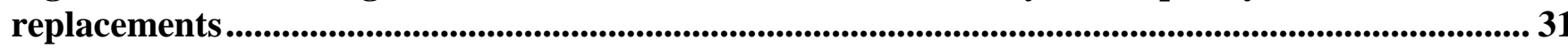




\section{INTRODUCTION ${ }^{2}$}

Internally enhanced tubes, like the micro-fin tube, are used by most manufacturers in the construction of evaporators and condensers for new unitary refrigeration and air-conditioning equipment. The reason for the micro-fin tube's hold on unitary equipment is that it provides the highest heat transfer with the lowest pressure drop of the commercially available internal enhancements (Webb and Kim, 2005). Most of the experimental measurements for evaporative heat transfer coefficients in the micro-fin tube have been done for traditional refrigerants like R134a. Pressure from the policies set by the Montreal Protocol (1987), the Kyoto Protocol (1997) and the European Mobile Directive (2006) have caused a recent shift to refrigerants with both zero ozone depletion potential (ODP) and low global warming potential (GWP). Although two current workhorse refrigerants R410A and R404A, for air-conditioning and refrigeration respectively, have zero ODP, they both have rather large GWP: 1725 and 3300, respectively (IPCC, 2013). Three new refrigerants, R448A, R449A, and R452B, are potential low GWP replacements for R404A (R125/R143A/R134A (44/52/4)). ${ }^{3}$ In addition, R452B is also a proposed replacement for R410A (R32/R125 (50/50)). R448A, R449A, and R452B are of interest as replacements because they have zero ODP and 100 year GWPs of approximately 1274 (IPCC, 2013), 1283 (Minor et al., 2015), and 676 (Minor, 2016), respectively. Consequently, flow boiling heat transfer data for the micro-fin tube with R448A (R32/R125/R134a/R1234ze/R1234yf (26/26/21/7/20)), R449A (R32/R125/R134a/R1234yf (24.3/24.7/25.7/25.3)), and R452B ${ }^{4}$ (R32/ R125/R1234yf (67/7/26)) are essential for the evaluation of their use for unitary applications.

The bulk of the research on flow boiling in micro-fin tubes has been for well-established alternative refrigerants with GWP greater than 1200. For example, Targanski and Cieslinski (2007) measured the evaporation heat transfer characteristics of R407C inside a micro-fin tube in the presence of oil. Zhang et al. (2007, 2015) measured the evaporation heat transfer coefficients of R417A and R22 inside a micro-fin tube and introduced a new heat transfer correlation to predict their values. Yun et al. (2002) examined existing experimental data and developed a model, which was validated for use with R22, R113, R123, R134a, and R410A and a variety of micro-fin tube geometries. Seo et al. (2000), Yu et al. (2002), and Kim et al. (2002) measured the flow boiling heat transfer coefficient in micro-fin tubes for R22, R134a, and R410A, respectively. Wellsandt and Vamling (2005) have investigated in-tube evaporation of R134a in a special type of micro-fin tube where the fin rifling, instead of being continuous, is arranged into $\mathrm{V}$-grooves that resemble herringbones; hence, it is called the herringbone micro-fin tube. Oliver et al. (2004) have also studied the two-phase heat transfer performance of a herringbone and a standard 18-deg helical micro-fin tube with R22, R134a and R407C.

Recent research on alternative refrigerants with GWP less than 1300 have been done for smooth tubes. For example, Grauso et al. (2013), and Hossain et al. (2013) have provided flow boiling studies with $\mathrm{R} 1234 z e(E)$ in horizontal smooth tubes. Three studies used electrically heated test rigs to measure the local flow boiling heat transfer coefficient in smooth tubes. The first, Meng et al. (2013), tested R141b in vertical and inclined serpentine tubes. The second, Nasr et al. (2015), tested R600a in a horizontal plain tube. The third, Zou et al. (2010), tested two different R290/R152a mixtures in a horizontal tube.

\footnotetext{
${ }^{2}$ Certain trade names and company products are mentioned in the text or identified in an illustration in order to adequately specify the experimental procedure and equipment used. In no case does such an identification imply recommendation or endorsement by the National Institute of Standards and Technology, nor does it imply that the products are necessarily the best available for the purpose.

3 All compositions are by mass.

${ }^{4}$ R452B is the pending ASHRAE designation for the mixture R32/ R125/R1234yf (67/7/26), which has the developmental designation DR-55.
} 
Most if not all of the relatively recent research on flow boiling in micro-fin tubes for refrigerants having GWP less than 1300 has been done for R134a and R410A replacements rather than for those intended to replace R404A. For example, Mendoza-Miranda et al. (2015) and Diani et al. (2015) have experimentally investigated the heat transfer performance of R1234yf in micro-fin tubes. Diani et al. (2015) made measurements on a single tube and found that they compared well with heat transfer correlations from the literature. Mendoza-Miranda et al. (2015) measured the overall heat transfer performance of a shell-and-tube heat exchanger in an operating refrigerant cycle. They used micro-fin tube correlations from the literature and found that the heat transfer performance of a shell-and-tube heat exchanger with micro-fin tubes could be successfully modeled. Kondou et al. (2013) measured the flow boiling heat transfer coefficient of R32/R1234ze(E) and its components in a horizontal micro-fin tube, and they developed a new correlation to predict their zeotropic mixture heat transfer coefficients.

Because of the relatively recent introduction of R448A, R449A, and R452B, measured heat transfer data in a micro-fin tube are not available in the literature for these refrigerants. Consequently, the present study provides measured local flow boiling heat transfer for these three low-GWP refrigerants in a micro-fin tube for test conditions that are applicable for air conditioning applications.

\section{EXPERIMENTAL APPARATUS}

Figure 1 shows a sketch of the experimental apparatus used to establish and measure convective boiling heat transfer coefficients. The experimental test facility consisted of two main systems: the refrigerant loop and water loop. The refrigerant flow rate, pressure, and superheat were fixed at the inlet to the test section. The water flow rate and the inlet temperature were fixed to establish the overall refrigerant quality change in the test section. The water temperature drop, the tube wall temperature, the refrigerant temperatures, pressures, and pressure drops were measured at several axial locations along the test section. These measurements were used to calculate the local heat-transfer coefficient for the micro-fin tube.

The test section consisted of a pair of $3.34 \mathrm{~m}$ long, horizontal tubes connected by a U-bend. A fixed test pressure was maintained by balancing the refrigerant duty between the subcooler, the test section, and the evaporator. A magnetically coupled gear pump delivered the test refrigerant to the entrance of the test section with a few degrees of vapor superheat. Another magnetically coupled gear pump supplied a steady flow of water to the annulus of the test section. The inlet temperature of the water loop was held constant for each test with a water chilled heat exchanger and variable electric heaters. The refrigerant and water flow rates were controlled by varying the pump speeds using frequency inverters. Redundant flow rate measurements were made with Coriolis flowmeters and with turbine flowmeters for both the refrigerant and water sides.

Figure 2 shows a cross section of the test section with a detail of the micro-fin tube geometry. The test refrigerant flowed inside a micro-fin tube, while distilled water flowed either in parallel flow or counterflow to the refrigerant in the annulus that surrounded the micro-fin tube. Having some tests in parallel flow and others in counterflow produced a broad range of heat fluxes at both low and high flow qualities. The annulus gap was $2.2 \mathrm{~mm}$, and the micro-fin tube wall thickness was $0.3 \mathrm{~mm}$. The micro fin tube had 60 , $0.2 \mathrm{~mm}$ high fins with an 18 degree helix angle. For this geometry, the cross sectional flow area was $60.8 \mathrm{~mm}^{2}$, giving an equivalent smooth diameter $\left(D_{e}\right)$ of $8.8 \mathrm{~mm}$. The root diameter of the micro-fin tube was $8.91 \mathrm{~mm}$. The inside-surface area per unit length of the tube was estimated to be $44.6 \mathrm{~mm}$. The hydraulic diameter $\left(D_{h}\right)$ was measured with a polar planimeter from a scaled drawing of the tube cross 
section and determined to be approximately $5.45 \mathrm{~mm}$. The ratio of the inner surface area of the micro fin tube to the surface area of a smooth tube of the same $D_{e}$ was 1.6. The fins rifled down the axis of the tube at a helix angle of $18^{\circ}$ with respect to the tube axis.

Figure 3 provides a detailed schematic of the test section. The annulus was constructed by connecting a series of tubes with 14 pairs of stainless steel flanges. This construction permitted the measurement of both the outer micro-fin wall temperature and the water temperature drop as discussed in the following two paragraphs. The design also avoided abrupt discontinuities such as unheated portions of the test section and tube-wall "fins" between thermopile ends.

Figure 3 shows that thermocouple wires pass between 12 of the gasketed flange pairs to measure the refrigerant-tube wall temperature at ten locations on the top, side, and bottom of the tube wall. These locations were separated by $0.6 \mathrm{~m}$ on average, and they were located near the intersection of the shell flanges. In addition to these, thermocouples were also mounted next to the pressure taps near the middle of each test section length. The thermocouple junction was soldered to the outside surface and was sanded to a thickness of $0.5 \mathrm{~mm}$. The leads were strapped to a thin non-electrically-conducting epoxy layer on the wall for a distance of $14.3 \mathrm{~mm}$ before they passed between a pair of the shell flanges. The wall temperature was corrected for a heat flux dependent fin effect. The correction was typically $0.05 \mathrm{~K}$. Figure 3 also shows that a chain of thermopiles was used to measure the water temperature drop between each flange location. Each thermopile consisted of ten thermocouples in series, with the ten junctions at each end evenly spaced around the circumference of the annulus. Because the upstream junctions of one thermopile and the downstream junctions of another enter the annulus at the same axial location (except at the water inlet and outlet), the junctions of the adjacent piles were alternated around the circumference. A series of Teflon half-rings attached to the inner refrigerant tube centered the tube in the annulus. The half-rings were circumferentially baffled to mix the water flow. Mixing was further ensured by a turbulent water Reynolds number (Kattan et al. 1995).

As shown in Fig. 3, six refrigerant pressure taps along the test section allowed the measurement of the upstream absolute pressure and five pressure drops along the test section. Two sets of two water pressure taps were used to measure the water pressure drop along each tube. Also, a sheathed thermocouple measured the refrigerant temperature at each end of the two refrigerant tubes, with the junction of each centered radially. Only the thermocouple at the inlet of the first tube was used in the calculations. The entire test section was wrapped with $5 \mathrm{~cm}$ of foam insulation to minimize heat transfer between the water and the ambient.

\section{MEASUREMENTS}

Table 1 shows the expanded measurement uncertainty $(U)$ of the various measurements along with the range of each test parameter in this study. The $U$ was estimated with the law of propagation of uncertainty. All expanded measurement uncertainties are reported at the $95 \%$ confidence level. The estimates shown in Table 1 are median values of $U$ for the correlated data. Saturated refrigerant properties were evaluated at the measured saturation pressure with the REFPROP (Lemmon et al. 2013) equation of state while using refrigerant-vendor proprietary fluid files. Table 2 shows representative properties that were obtained from REFPROP for the fluids germane to this study. The liquid dynamic viscosity as calculated with REFPROP for R449A did not agree with that provided by the fluid vendor. ${ }^{5}$ The left side of Table 2 shows properties

\footnotetext{
${ }^{5}$ For this case, the following equation was used, which was obtained from a fit the vendor data for the temperature range of
} 
that were evaluated for the average test conditions of each fluid, while the right side of the table provides properties that were evaluated at $277.6 \mathrm{~K}$.

The convective boiling heat transfer coefficient based on the actual inner surface area $\left(h_{2 \phi}\right)$ was calculated as:

$$
h_{2 \phi}=\frac{q^{\prime \prime}}{T_{\mathrm{w}}-T_{\mathrm{s}}}
$$

where the measured wall temperatures $\left(T_{\mathrm{w}}\right)$ were fitted to their axial position to reduce the uncertainty in the measurement.

Figure 4 shows the estimated expanded uncertainty of the wall temperature fit for all the measurements as a function of thermodynamic quality. Figure 4 includes some data that was omitted from the correlation as explained in the Results Section. The uncertainty of roughly $90 \%$ of the fitted wall temperatures was less than $0.5 \mathrm{~K}$ at the $95 \%$ confidence level. The median of the uncertainty in $T_{\mathrm{w}}$ as shown in Table 1 was approximately $0.3 \mathrm{~K}$.

The water temperature $\left(T_{\mathrm{f}}\right)$ was determined from the measured temperature change obtained from each thermopile and the inlet water temperature measurement. The water temperature gradient $\left(\mathrm{d} T_{\mathrm{f}} / \mathrm{dz}\right)$ was calculated with second-order finite difference equations using the measured water temperatures and their locations along the tube length $z$. The water temperature gradients were then fitted with respect to the tube length. As a check on the water temperature gradient calculation, Fig. 5 shows that the measured water temperatures (open circles) typically agreed with the integrated fit of the water temperature gradient (solid line) to within $0.2 \mathrm{~K}$.

The fitted, local, axial water temperature gradient $\left(\mathrm{d} T_{\mathrm{f}} / \mathrm{d} z\right)$, the measured water mass flow rate $\left(\dot{m}_{\mathrm{f}}\right)$, and the properties of the water were used to calculate the local heat flux $\left(q^{\prime \prime}\right)$ to the micro-fin tube based on the actual inner surface area:

$$
q^{\prime \prime}=\frac{\dot{m}_{\mathrm{f}}}{p}\left(c_{\mathrm{p}_{\mathrm{f}}} \frac{\mathrm{d} T_{\mathrm{f}}}{\mathrm{d} z}+v_{\mathrm{f}} \frac{\mathrm{d} P_{\mathrm{f}}}{\mathrm{d} z}\right)
$$

where $p$ is the wetted perimeter of the inside of the micro-fin tube. The specific heat $\left(c_{\mathrm{pf}}\right)$ and the specific volume $\left(v_{\mathrm{f}}\right)$ of the water were calculated locally as a function of the water temperature. The water pressure gradient $\left(d P_{f} / d z\right)$ was linearly interpolated between the pressure taps to the location of the wall thermocouples. The pressure gradient term was typically less than $3 \%$ of the temperature gradient term. The heat flux obtained by eq. (2) was reduced by the amount of heat lost to the surroundings. The heat loss to the surroundings was obtained by calibration of single phase heat transfer tests and it was based on the temperature difference between the room and the test fluid. Typically, the heat loss correction was less than a $0.1 \%$ correction of eq. (2). Figure 6 plots the relative uncertainty of the heat flux measurement versus thermodynamic quality. As shown in Fig. 6, the uncertainty of the heat flux remains less than $40 \%$ of the

this study: $\mu_{\mathrm{r}}=4548.61-38.4351 T_{r}+0.114838 T_{\mathrm{r}}^{2}-0.119168 \times 10^{-3} T_{\mathrm{r}}^{3}$ where $\mu_{\mathrm{r}}$ and $T_{\mathrm{r}}$ have units of $\mu \mathrm{Pa}$ s and K, respectively. 
measured value, while the average uncertainty for the counterflow and the parallel flow data is approximately $8 \%$ and $19 \%$ of the measured value, respectively.

Figure 7 shows example plots of the local heat flux as calculated from eq. (2) versus thermodynamic quality for both cases when the water and the refrigerant are in counterflow and parallel flow, respectively. Both heat flux profiles are for R449A at an all-liquid Reynolds number (Re) of roughly 6800 and a refrigerant reduced pressure of approximately 0.16 , which was evaluated at the exit of the test section. The discontinuity exhibited in the heat flux profiles is due to the change in refrigerant saturation temperature as caused by the adiabatic pressure drop in the bend that is used to transition from the first leg of the test section to the second leg. The decrease in the refrigerant saturation temperature causes an increase in the difference between the water and the refrigerant temperature, which leads to an increase in the local heat flux. For the counterflow case, the heat flux increases from approximately $2 \mathrm{~kW} \cdot \mathrm{m}^{-2}$ at a quality near zero to approximately $12 \mathrm{~kW} \cdot \mathrm{m}^{-2}$ at a quality slightly greater than 0.7 . The parallel flow case has the opposite slope and a slightly different range of that for counterflow where the heat flux decreases from approximately $25 \mathrm{~kW} \cdot \mathrm{m}^{-2}$ at a quality near 0.11 to approximately $3 \mathrm{~kW} \cdot \mathrm{m}^{-2}$ at a quality of approximately 0.9 .

The thermodynamic and transport properties were calculated with version 9.1 of REFPROP (Lemmon et al. 2010) while using enthalpy and pressure as inputs. The enthalpy of the refrigerant liquid at the inlet of the test section was calculated from its measured temperature and pressure. The subsequent increase in refrigerant enthalpy along the test section was calculated from the local heat flux and the measured refrigerant mass flow rate. The refrigerant pressures were measured at six pressure taps along the test section. The pressure was linearly interpolated between the taps. The refrigerant entering the test section was near saturation and held to a saturation temperature of approximately $277.6 \mathrm{~K}$ for all of the tests. Considering that the tests were done for quality ranges between near zero and slightly greater than 0.7 , the saturation temperature of R448A and R449A both increased from roughly $277.6 \mathrm{~K}$ to $282.0 \mathrm{~K}$ for most tests. By comparison, the variation of the saturation temperature of R452B from the inlet temperature, as caused by the pressure drop and the small temperature glide, was generally less than $1 \mathrm{~K}$.

The local Nusselt number $(\mathrm{Nu})$ was calculated using the hydraulic diameter and the heat transfer coefficient based on the actual inner surface area of the tube as:

$$
\mathrm{Nu}=\frac{h_{2 \phi} D_{h}}{k_{l}}
$$

Figure 8 shows the relative uncertainty of the $\mathrm{Nu}$ versus thermodynamic quality was between roughly $10 \%$ and $40 \%$. Measurements of $\mathrm{Nu}$ with uncertainties greater than $40 \%$ were discarded. The average uncertainty of $\mathrm{Nu}$ for presented data was approximately $25 \%$ for all qualities.

\section{RESULTS}

The 816 data points generated in this study for R448A, R449A, and R452B are tabulated in Appendix A, which contains the Nusselt and all-liquid Reynolds numbers and other reduced data that are typically used to characterize flow boiling. Appendix B contains the raw data measurements including the heat flux and the wall and water temperatures and locations. The column entitled, "flow," provides a "C" or a "P" to indicate that the measurements were made for either counterflow or parallel flow between the refrigerant and water, respectively. All the parameters given in Appendix A and B are defined in the Nomenclature.

The measured local convective boiling Nusselt numbers $(\mathrm{Nu})$ were compared to the pure-refrigerant (single 
component) version of the Hamilton et al. (2008) correlation:

$$
\mathrm{Nu}_{\mathrm{p}}=482.18 \operatorname{Re}^{0.3} \operatorname{Pr}^{\mathrm{C}_{1}}\left(\frac{P_{\mathrm{s}}}{P_{\mathrm{c}}}\right)^{\mathrm{C}_{2}} \mathrm{Bo}^{\mathrm{C}_{3}}\left(-\log _{10} \frac{P_{\mathrm{s}}}{P_{\mathrm{c}}}\right)^{\mathrm{C}_{4}} M_{\mathrm{w}}{ }^{\mathrm{C}_{5}}
$$

where

$$
\begin{aligned}
& C_{1}=0.51 x_{q} \\
& C_{2}=5.57 x_{q}-5.21 x_{q}^{2} \\
& C_{3}=0.54-1.56 x_{q}+1.42 x_{q}^{2} \\
& C_{4}=-0.81+12.56 x_{q}-11.00 x_{q}^{2} \\
& C_{5}=0.25-0.035 x_{q}^{2}
\end{aligned}
$$

Here, the all-liquid Reynolds number (Re), the Boiling number (Bo), the liquid Prandtl number $(\operatorname{Pr})$, the reduced pressure $\left(P_{\mathrm{s}} / P_{\mathrm{c}}\right)$, and the quality $\left(x_{\mathrm{q}}\right)$ are all evaluated locally at the saturation temperature. The allliquid Reynolds number and the Nusselt number are based on the hydraulic diameter $\left(D_{\mathrm{h}}\right)$. The Nusselt number is also based on the actual inner surface area of the tube.

Hamilton et al. (2008) also provide a correction factor for eq. (4) to predict the heat transfer coefficient for binary mixtures, which cannot be used for mixtures of more than two components. Consequently, a new correction for eq. (4) was developed to predict the flow boiling Nusselt Number ( $\mathrm{Nu}$ ) for mixtures of any number of refrigerants. This was done by multiplying the single-component Nusselt Number $\left(\mathrm{Nu}_{\mathrm{p}}\right)$ by a modifier to predict multi-component mixtures:

$$
\mathrm{Nu}=\mathrm{Nu}_{\mathrm{p}}\left(1-36.23\left[\frac{T_{\mathrm{d}}-T_{\mathrm{b}}}{T_{\mathrm{b}}}\right] e^{-0.007 \mathrm{ReBo}^{0.47}}\right)
$$

where $T_{\mathrm{d}}$ and $T_{\mathrm{b}}$ are the dew point and bubble point temperatures, respectively, evaluated at the local saturation pressure and overall composition of the mixture. The $T_{\mathrm{d}}-T_{\mathrm{b}}$ difference is commonly called the temperature glide of the mixture. Typically, large temperature glides cause concentration gradients that lead to heat transfer degradations as compared to what would be expected from a single component prediction model (Kedzierski et al., 1992). Consequently, the bracketed term in eq. (5) that multiplies $\mathrm{Nu}_{\mathrm{p}}$ describes the mixture degradation effect, which is a function of temperature glide, Bo and Re. A single component refrigerant would have zero temperature glide, which would result in the mixture degradation effect, represented by the bracketed term, being equal to one.

The flow map of Yu et al. (2002) for micro-fin tubes was used to determine that approximately $87 \%$ of the measurements were in annular or semi-annular flow. Manwell and Bergles (1990) suggest that the reason annular-like flow is a strong characteristic of micro-fin tubes is that the spiraling fins along the tube axis encourage wetting of the upper tube wall. 
Figure 9 shows a comparison between the boiling Nusselt numbers predicted with eq. (5) for the micro-fin tube to those measured here for R448A, R449A, and R452B. Equation (5) predicts approximately $98 \%$ of the measured convective boiling Nusselt numbers for R448A, R449A, and R452B in the micro-fin tube to within approximately $\pm 20 \%$. The measurements for each fluid are roughly centered about the mean of the correlation suggesting a lack of bias in the prediction due to the different fluids or some other cause. The gray dashed lines are multi-use $95 \%$ confidence intervals on the mean prediction, which vary from $\pm 16 \%$ of the prediction at a $\mathrm{Nu}$ of approximately 50 to less than $\pm 5 \%$ for Nusselt numbers larger than 100 . If the uncertainty in the fluid properties are neglected and the model is assumed to be correct, then the source for the uncertainty of the mean resides entirely in the uncertainty of the fitted constants. If this is true, then the uncertainty in the ratio of the heat transfer coefficients as predicted with eq. (5) for two different fluids is no greater than the uncertainty of the mean for eq. (5) as shown in Fig. 9 for the $95 \%$ confidence level, which is less than $\pm 5 \%$ for Nusselt numbers larger than 100 .

Representative plots of the heat transfer coefficient $\left(h_{2 \phi}\right)$ versus thermodynamic quality $\left(x_{q}\right)$ are given in Figs. 10 and 11. The solid lines are predictions for the present micro-fin tube geometry, which were obtained from the modified Hamilton et al. (2008) correlation given in eq. (5). The symbols are the measured data points, while the shaded regions between the dashed lines provide the measurement uncertainty for a $95 \%$ confidence level. The uncertainty in the R448A and R449A heat transfer coefficients is shown to be roughly $500 \mathrm{~W} \cdot \mathrm{K}^{-1} \cdot \mathrm{m}^{-2}$ for most of data for qualities greater than $20 \%$. For these measurements, the uncertainty in the heat flux is the greatest contributor to the uncertainty in the heat transfer coefficient. The uncertainty in the R452B heat transfer coefficient is approximately three times larger than that of the other refrigerants for this particular example and is shown to be roughly $1500 \mathrm{~W} \cdot \mathrm{K}^{-}$ ${ }^{1} \cdot \mathrm{m}^{-2}$ for most of data for qualities greater than $20 \%$. This is primarily due to the larger heat transfer coefficients measurements. More specifically, because of the larger heat transfer coefficients, and with it, the associated smaller driving temperature difference, the uncertainty in the heat flux and the uncertainty in the driving temperature difference contribute nearly equally to the uncertainty in the heat transfer coefficient, resulting in a larger total uncertainty.

Figure 10 shows the local heat transfer coefficient for R448A, R449A, and R452B for $G_{\mathrm{r}} \sim 100 \mathrm{~kg} \mathrm{~s}^{-1} \mathrm{~m}^{-2}$ and $P_{\mathrm{s}} / P_{\mathrm{c}} \sim 0.16\left(T_{\mathrm{s}} / T_{\mathrm{c}} \sim 0.79\right)$ with counterflow between the refrigerant and the water. The $P_{\mathrm{s}} / P_{\mathrm{c}}$ ratio is evaluated at the exit of the test section. As shown in Fig. 7, the counterflow condition provided increasing heat flux with increasing thermodynamic quality. As the convective boiling heat transfer coefficient is moderately dependent upon the heat flux, the increasing heat flux and the thinning liquid films on the wall cause the heat transfer coefficient to increase with respect to quality. Equation 5 is shown to predict both the R452B and the R449A measurements to within approximately $100 \mathrm{~W} \cdot \mathrm{K}^{-1} \cdot \mathrm{m}^{-2}$, i.e., approximately $\pm 3 \%$ and $\pm 5 \%$, respectively. The maximum deviation between the eq. (5) predictions and the measurements for $\mathrm{R} 448 \mathrm{~A}$ is larger for this case being approximately $200 \mathrm{~W} \cdot \mathrm{K}^{-1} \cdot \mathrm{m}^{-2}$, or approximately $\pm 12 \%$. Overall, the average difference between the measurements and the predictions for all of the counterflow measurements for all the test fluids is approximately $\pm 7 \%$.

Figure 11 shows the local heat transfer coefficient for R448A, R449A, and R452B for $G_{\mathrm{r}} \sim 200 \mathrm{~kg} \mathrm{~s}^{-1} \mathrm{~m}^{-2}$ and $P_{\mathrm{s}} / P_{\mathrm{c}} \sim 0.16\left(T_{\mathrm{s}} / T_{\mathrm{c}} \sim 0.79\right)$ with parallel flow between the refrigerant and the water. As shown in Fig. 7, the parallel flow condition provided decreasing heat flux with increasing thermodynamic quality. Because of the high heat flux for qualities less than $20 \%$, it is likely that nucleate boiling may be a larger contribution to the total heat transfer than it is for the counterflow condition, thus contributing to the large 
heat transfer coefficient the low quality region. For qualities larger than $20 \%$, the effects of decreasing heat flux and the thinning liquid films on the wall cause the heat transfer coefficient to moderately decrease with respect to quality. Equation 5 is show to predict both the R449A and the R449B measurements to within approximately $200 \mathrm{~W} \cdot \mathrm{K}^{-1} \cdot \mathrm{m}^{-2}$,i.e., approximately $\pm 5 \%$, for qualities less than 0.7 . The maximum deviation between the eq. (5) predictions and the measurements for R452B is larger for this case being approximately $500 \mathrm{~W} \cdot \mathrm{K}^{-1} \cdot \mathrm{m}^{-2}$, or approximately $\pm 9 \%$. Overall, the average difference between the measurements and the predictions for all of the parallel flow measurements for all the test fluids is approximately $\pm 10 \%$.

The main purpose of Figs. 10 and 11 was to compare the eq. (5) predictions to the measurements for each fluid at as similar as conditions as the present data set would allow. Even though the measurements were compared at nearly the same mass velocity, $100 \mathrm{~kg} \mathrm{~s}^{-1} \mathrm{~m}^{-2}$ for Fig. 10 and $200 \mathrm{~kg} \mathrm{~s}^{-1} \mathrm{~m}^{-2}$ for Fig. 11, the local heat flux could vary significantly between fluids. For the Fig. 10 example, the heat flux for R449A varied from near zero to approximately $4.5 \mathrm{~kW} \cdot \mathrm{m}^{-2}$, while that for R448A varied from approximately $1.5 \mathrm{~kW} \cdot \mathrm{m}^{-2}$ to $3.2 \mathrm{~kW} \cdot \mathrm{m}^{-2}$. This illustrates that the maximum heat flux for the R449A counterflow data set example was approximately $41 \%$ larger than that for R448A, which accentuated the difference in the measured heat transfer coefficients between the two fluids. For this reason, it is important to use a validated model to compare the performance of the fluids at identical conditions in order to establish a fair comparison of relative fluid heat transfer performance.

Figure 12 uses eq. (5) to illustrate the relative heat transfer performance of R448A, R449A, and R452B versus quality for the same saturated refrigerant inlet temperature $\left(T_{\mathrm{r}, \mathrm{i}}=277.6 \mathrm{~K}\right)$, and the same refrigerant mass flux $\left(G_{\mathrm{r}}=100 \mathrm{~kg} \cdot \mathrm{m}^{-2} \cdot \mathrm{s}^{-1}\right)$ for the present micro-fin tube geometry. Both counterflow and parallel flow conditions are shown. The counterflow heat flux is approximated with $q^{\prime \prime}=15.1 x_{\mathrm{q}}^{0.51} \mathrm{~kW} \cdot \mathrm{m}^{-2}$, while the parallel flow is approximated with $q^{\prime \prime}=\left(29.6-29.8 x_{q}\right) \mathrm{kW} \cdot \mathrm{m}^{-2}$. The heat flux profiles with respect to quality that were used to calculate the heat transfer coefficient are approximately equivalent to those shown in Fig. 7. Five different line styles for each flow condition are used to represent the predictions for the three different test fluids and two baseline refrigerants, R410A and R404A, as labeled.

In general, for counterflow, Fig. 12 predictions show that the boiling heat-transfer coefficient rapidly increases with increasing quality for qualities less than $20 \%$. For quality ranges between $20 \%$ and $70 \%$, the rate of increase in the heat transfer coefficient with respect to increasing quality is roughly a fourth of that for qualities less than $20 \%$. For parallel flow, the heat transfer coefficient for all the fluids decreases with increasing quality in response to the decreasing heat flux with respect to increasing quality. For the example case presented here, the heat transfer coefficient for R449A is approximately $8 \%$ larger than the heat transfer coefficient for R448A for qualities between $10 \%$ and $70 \%$ for both counterflow and parallel flow conditions. For both counterflow and parallel flow, the heat transfer coefficients for R449A and R448A are roughly $1.5 \mathrm{~kW} \cdot \mathrm{K}^{-1} \cdot \mathrm{m}^{-2}$ less than that of R404A for qualities between $10 \%$ and $70 \%$. R449A has a larger heat transfer coefficient than R448A due partly to its $2 \%$ larger thermal conductivity and its $6 \%$ smaller temperature glide as illustrated in Table 2. As a percentage, the heat transfer coefficients for R449A and R448A are $26 \%$ to $43 \%$ and $31 \%$ to $48 \%$, respectively, less than that of R404A. The smaller heat transfer coefficient of the alternative refrigerants as compared to that of R404A is primarily due to the larger mixture degradation effect as calculated by the bracketed right-side of eq. (5) being on average 0.61, 0.63, and 0.97 for R449A, R448A, and R404A, respectively. This degradation factor reduces the single component prediction by an amount that is sufficient to account for the mixture degradation effects. A 
value of 0.97 for R404A indicates that the flow boiling performance of R404A closely matches (within $3 \%$ of) that of a single component refrigerant because of its negligible temperature glide. In addition, R404A has a $21 \%$ larger Prandtl number than either R449A or R448A, which also contributes to the favorable performance of R404A as compared to the two low-GWP refrigerants examined here.

The $8 \%$ difference in heat transfer performance between R449A and R448A may not significantly affect the cycle performance of an air conditioner because the air-side heat transfer resistance typically dominates the overall resistance of a refrigerant-to-air, air-conditioning evaporator. As a result, the change in the overall conductance of the evaporator would be limited to some fraction of the $8 \%$ change in refrigerantside heat transfer. In addition, the uncertainty of the heat transfer ratio prediction for this case is approximately $\pm 5 \%$, which puts the range of possible overall difference roughly between $3 \%$ and $13 \%$ for $95 \%$ confidence. Finally, the heat transfer performance shown in Fig. 12 is only a representative performance, which is likely to change for differing conditions such as different mass velocities. However, because R449A has a $2 \%$ larger thermal conductivity and a $6 \%$ smaller temperature glide as compared to R448A, with other salient fluid properties being nearly the same, it is not surprising that the present study has shown R449A to have heat transfer coefficients that are marginally larger than those of R448A for the example case.

Figure 12 also shows the predicted relative performance of R452B to the other new replacements and also to the existing refrigerants R404A and R410A. Because of its large liquid thermal conductivity (29\%, $27 \%$, and $54 \%$ larger than R448A, R449A, and R404A, respectively) and its negligible temperature glide, which results in nearly single component heat transfer performance ( 0.92 mixture degradation factor), the heat transfer performance of R452B is significantly larger than R404A, R448A, and R449A. Averaged for qualities between $10 \%$ and $70 \%$ as presented in Fig. 12 for counterflow, the heat transfer coefficient is approximately $90 \%, 70 \%$, and $13 \%$ larger than that of R448A, R449A, and R404A, respectively. A similar comparison results for parallel flow: the average heat transfer coefficient for $\mathrm{R} 452 \mathrm{~B}$ is approximately $73 \%, 59 \%$, and $14 \%$ larger than that of R448A, R449A, and R404A, respectively. As a R410A replacement, R452B closely matches the performance of R410A being approximately within $10 \%$ of the R410A heat transfer coefficient for qualities between $10 \%$ and $70 \%$. On average, the heat transfer coefficient for R452B is predicted to be approximately $5 \%$ less than that of R410A for qualities between $10 \%$ and $70 \%$ for both flow conditions.

According to Minor (2016), R407A and R407F are replacements currently used for R404A. Considering this, additional predictive calculations were made using eq. (5) for R407A and R407F for the same conditions as were used for the above-discussed predictions and are presented in Fig. 13. The results showed that the heat transfer coefficients for R407A and R407F reside between that of R448A and R449A, with the heat transfer coefficient of $\mathrm{R} 407 \mathrm{~F}$ being no more than $100 \mathrm{~W} \cdot \mathrm{K}^{-1} \cdot \mathrm{m}^{-2}$ greater than that for R407A. The difference between the R449A and the R448A heat transfer coefficients remains within $300 \mathrm{~W} \cdot \mathrm{K}^{-1} \cdot \mathrm{m}^{-2}$ for both counterflow and parallel flow for all qualities for the example shown in Fig. 13. This difference, on average, is approximately $10 \%$ of the predicted heat transfer coefficient. By comparison, the heat transfer coefficient of R407A and R407F are roughly, on average, within $5 \%$ of that of either R449A or R448A.

\section{CONCLUSIONS}

Local convective boiling heat transfer measurements for three low-GWP refrigerants in a fluid heated micro-fin tube were presented. The measured convective boiling Nusselt numbers for all of the test 
refrigerants were compared to an existing correlation from the literature that was modified to predict multicomponent mixtures. Approximately, $98 \%$ of the measurements were predicted to within $\pm 20 \%$ and centered about the mean prediction.

In general, the measured boiling heat-transfer coefficient increased with increasing qualities for counterflow between the refrigerant and the water. In contrast, for parallel flow, the measured heat transfer coefficient was relatively constant with respect to quality. The heat transfer coefficient of the three test fluids were compared at the same heat flux, saturated refrigerant temperature, and refrigerant mass flux by using the modified correlation from the literature. The resulting comparison showed that refrigerant R452B exhibited the highest heat transfer performance of the three low-GWP refrigerants in large part due to its higher thermal conductivity and small temperature glide. For the example case presented here, the heat transfer coefficient for R449A was approximately $8 \%$ larger than the heat transfer coefficient for R448A for qualities between $10 \%$ and $70 \%$. The heat transfer coefficient for R452B was predicted to be, on average, approximately $73 \%$ to $90 \%$ larger than R448A and approximately $59 \%$ to $70 \%$ larger than R449A. The heat transfer coefficients for R449A and R448A are $26 \%$ to $43 \%$ and $31 \%$ to $48 \%$, respectively, less than that of R404A for qualities between $10 \%$ and $70 \%$. The smaller heat transfer coefficient of R448A and R449A, as compared to that of R404A, results despite having an approximate $20 \%$ larger liquid thermal conductivity than R404A. In contrast, the model predicts that the R452B heat transfer coefficient is approximately $13 \%$ larger than that of R404A for thermodynamic qualities between $10 \%$ and $70 \%$.

\section{ACKNOWLEDGEMENTS}

This work was funded by NIST. The authors thank the following for their constructive criticism of the first draft of the manuscript: Dr. P. Domanski, and Dr. D. Veronica from NIST, and Dr. S. Treado from The Pennsylvania State University. Thanks also go to Wolverine Tube, Inc., for supplying the Turbo-A, microfin tube for the test section, and to Chemours and Honeywell for supplying the test refrigerants and the equation of state parameters for REFPROP. 


\section{NOMENCLATURE}

English symbols

$A_{\mathrm{c}} \quad$ cross-sectional area $\left(\mathrm{m}^{2}\right)$

Bo local boiling number, $\frac{q^{\prime \prime}}{G_{r} i_{f g}}$

$c_{\mathrm{p}} \quad$ specific heat $\left(\mathrm{J} \mathrm{kg}^{-1} \mathrm{~K}^{-1}\right)$

$C \quad$ coefficients given in eq. (5)

$D_{\mathrm{e}} \quad$ equivalent inner diameter of smooth tube, $\sqrt{\frac{4 A_{\mathrm{c}}}{\pi}}(\mathrm{m})$

$D_{\mathrm{h}} \quad$ hydraulic diameter of micro-fin tube (m)

$e \quad$ fin height (mm)

$G \quad$ total mass velocity $\left(\mathrm{kg} \mathrm{m}^{-2} \mathrm{~s}^{-1}\right)$

$h_{2 \phi} \quad$ local two-phase heat-transfer coefficient $\left(\mathrm{W} \mathrm{m}^{-2} \cdot \mathrm{K}^{-1}\right)$

$i_{\text {fg }} \quad$ latent heat of vaporization $\left(\mathrm{J} \mathrm{kg}^{-1}\right)$

$k \quad$ refrigerant thermal conductivity $\left(\mathrm{W} \mathrm{m}^{-1} \mathrm{~K}^{-1}\right)$

$\mathrm{Nu} \quad$ local Nusselt number based on $D_{\mathrm{h}}$

$\dot{m} \quad$ mass flow rate $\left(\mathrm{kg} \mathrm{s}^{-1}\right)$

$M_{\mathrm{w}} \quad$ molar mass $\left(\mathrm{g} \mathrm{mole}^{-1}\right)$

$p \quad$ wetted perimeter $(\mathrm{m})$

$P \quad$ local fluid pressure $(\mathrm{Pa})$

Pr liquid refrigerant Prandtl number $\left.\frac{c_{p} \mu}{k}\right|_{r, l}$

$q^{\prime \prime} \quad$ local heat flux based on $A_{\mathrm{i}}\left(\mathrm{W} \mathrm{m}^{-2}\right)$

Re all liquid, refrigerant Reynolds number based on $D_{\mathrm{h}}=\frac{G_{r} D_{h}}{\mu_{r, l}}$

$S_{\mathrm{v}} \quad$ non-dimensional refrigerant specific volume given in Appendix A: $\frac{v_{\mathrm{v}}-v_{1}}{v}$

$s \quad$ distance between fins ( $\mathrm{mm})$

$T \quad$ temperature $(\mathrm{K})$

$t_{b} \quad$ bottom thickness of fin $(\mathrm{mm})$

$t_{w} \quad$ tube wall thickness (mm)

$U$ expanded relative uncertainty (\%)

$x_{\mathrm{q}} \quad$ thermodynamic mass quality (-)

$z \quad$ axial distance $(\mathrm{m})$

$\underline{\text { Greek symbols }}$

$\alpha \quad$ helix angle $\left({ }^{\circ}\right)$

$\beta \quad$ fin angle $\left(^{\circ}\right)$

$\Delta T_{\mathrm{s}} \quad T_{\mathrm{s}}-T_{\mathrm{w}}(\mathrm{K})$

$\mu \quad$ viscosity $(\mathrm{Pa} \cdot \mathrm{s})$

$v \quad$ specific volume, $\mathrm{x}_{\mathrm{q}} v_{\mathrm{v}}+\left(1-\mathrm{x}_{\mathrm{q}}\right) v_{1}\left(\mathrm{~m}^{3} \mathrm{~kg}^{-1}\right)$

$\rho \quad$ density $\left(\mathrm{kg} \mathrm{m}^{-3}\right)$

$\sigma \quad$ surface tension $\left(\mathrm{kg} \mathrm{s}^{-2}\right)$ 


\section{$\underline{\text { Subscripts }}$}

b bubble point

c critical condition

d dew point

f water

i inside, inlet

1 liquid

$\mathrm{p}$ prediction

$\mathrm{r}$ refrigerant

s saturated state

v vapor

w heat transfer surface 


\section{REFERENCES}

Diani, A., Mancin, S., and Rossetto, L. 2015. Flow boiling heat transfer of R1234yf inside a $3.4 \mathrm{~mm}$ ID microfin tube. Exp. Thermal Fluid Sci. 66:127-136.

Directive 2006/40/EC of The European Parliament \& of the Council of 17 May 2006 Relating to Emissions from Air-Conditioning Systems in Motor Vehicles \& Amending Council Directive 70/156/EC. Official Journal of the European Union, 49(L 161):12-18.

Grauso, S., Mastrullo, R., Mauro, A. W., Thome, J.R., and Vanoli, G. P. 2013 Flow pattern map, heat transfer and pressure drops during evaporation of R1234ze(e) and R134a in a horizontal, circular smooth tube: experiments and assessment of predictive methods. International Journal of Refrigeration 36:478491.

Hamilton, L. J., Kedzierski, M. A, and Kaul, M. P. 2008. Horizontal convective boiling of pure and mixed refrigerants within a micro-fin tube. Journal of Enhanced Heat Transfer 15(3):211-226.

Hossain, Md. A, Onaka, Y., Afroz, H., M.M., and Miyara, A. 2013. Heat transfer during evaporation of R1234ze(E), R32, R410A, and a mixture of R1234ze(E) and R32 inside a horizontal smooth tube. International Journal of Refrigeration 36:465-477.

IPCC 2013. Climate Change 2013: The Physical Science Basis. Contribution of Working Group I to the Fifth Assessment Report of the Intergovernmental Panel on Climate Change [Stocker, T.F., D. Qin, G.K. Plattner, M. Tignor, S.K. Allen, J. Boschung, A. Nauels, Y. Xia, V. Bex and P.M. Midgley (eds.)]. Cambridge University Press, Cambridge, United Kingdom and New York, NY, USA, 1535 pp, doi:10.1017/CBO9781107415324.

Kedzierski, M. A., and Kang, D. Y. 2016. Horizontal convective boiling of R448A, R449A, and R452B within a micro-fin tube with extensive measurement and analysis details. NIST Technical Note 2???. U.S. Department of Commerce, Washington, D.C.

Kedzierski, M. A., Kim, J. H., and Didion, D. A. 1992. Causes of the apparent heat transfer degradation for refrigerant mixtures. Two-Phase Flow and Heat Transfer, HTD-Vol. 197, J. H. Kim, R. A. Nelson, and A. Hashemi, Eds., ASME, New York, 197:149-158.

Kattan, N., Favret, D., and Thome, J. R. 1995. R-502 and two near-azeotropic alternatives: part 1 - in tube flow-boiling tests. ASHRAE Trans. 101(1):491-508.

Kim, Y., Seo, K., and Chung, J. 2002. Evaporation heat transfer characteristics of R-410A in 7 and 9.52 $\mathrm{mm} \mathrm{smooth} / \mathrm{micro}-$ fin tubes. International Journal of Refrigeration 25:716-730.

Kondou, C., BaBa, D., Mishima., F., and Koyama, S. 2013. Flow boiling of non-azeotropic mixture R32/R1234ze(E) in horizontal microfin tubes. International Journal of Refrigeration 36:2366-2378. Kyoto Protocol 1997. United Nations Framework Convention on Climate Change. United Nations (UN), New York, NY, USA. 
Lemmon, E. W., Huber, M. L., and McLinden, M. O. 2013. NIST Standard Reference Database 23, Version 9.1. Private Communications with McLinden, National Institute of Standards and Technology, Boulder, CO.

Manwell, S.P., and Bergles, A.E. 1990. Gas-liquid flow patterns in refrigerant-oil mixtures. ASHRAE Transactions 96(2):456-464.

Meng, M., Yang, Z., Duan, Y-Y, and Chen, Y. 2013. Boiling flow of R141b in vertical and inclined serpentine tubes. Int. J. Heat Mass Trans 57:312-320.

Mendoza-Miranda, J. M., Ramirez-Minguela, J.J., Munoz-Carpio, V.D., and Navarro-Esbri, J. 2015. Development and validation of a micro-fin tubes evaporator model using R134a and R1234yf as working fluids. International Journal of Refrigeration 50:32-43.

Minor, B., Gerstel, J., and Roberts, N. 2015. Evaluation of R-449A in field retrofits of R-404A supermarket systems. proceedings of the 24th International Congress of Refrigeration, Yokohama, Japan.

Minor, B. 2016. Private Communications, Chemours, Willmington, DE.

Montreal Protocol 1987. Montreal Protocol on Substances that Deplete the Ozone Layer. United Nations (UN), New York, NY, USA (1987 with subsequent amendments).

Olivier, J. A., Liebenberg, L., Kedzierski, M. A., and Meyer, J. P. 2004. Pressure drop during refrigerant condensation inside horizontal smooth, helical micro-fin, and herringbone micro-fin tubes. Journal of Heat Transfer 126:687-696.

Seo, K., and Kim, Y. 2000. Evaporation heat transfer and pressure drop of R-22 in 7 and $9.52 \mathrm{~mm}$ smooth/micro-fin tubes. International Journal of Heat and Mass Transfer 43:2869-2882.

Targanski, W., Cieslinski, J. T. 2007. Evaporation of R407C/oil mixtures inside corrugated and microfin tubes. Applied Thermal Engineering 27(13):2226-2232.

Webb, R. L., and Kim, N-H. 2005. Principles of Enhanced Heat Transfer, $2^{\text {nd }}$ ed., Taylor \& Francis, New York.

Wellsandt, S., and Vamling, L. 2005. Evaporation of R134a in a horizontal herringbone microfin tube: heat transfer and pressure drop. International Journal of Refrigeration 28:889-900.

Yu, M., Lin, T., and Tseng, C. 2002 Heat transfer and flow pattern during two-phase flow boiling of R134a in horizontal smooth and micro-fin tubes. International Journal of Refrigeration 25:789-798.

Yun, R., Kim, Y., Seo, K., and Kim, H. 2002. A generalized correlation for evaporation heat transfer of refrigerants in micro-fin tubes. International Journal of Heat and Mass Transfer 45:2003-2010. 
Zhang, X., Zhang, X., and Yuan, X. 2007. Evaporation heat transfer coefficients of R417a in horizontal smooth and microfin tubes," International Congress of Refrigeration, Beijing, ICR07-B1-228.

Zhang, X., Zhang, J., Ji, H., and Zhao, D. 2015. Heat transfer enhancement and pressure drop performance for R417A flow boiling in internally grooved tubes. Energy 86:446-454.

Zou, X., Gong, M., Chen, G., Sun, Z., and Wu, J. 2010. Experimental study on saturated flow boiling heat transfer of R290/R152a binary mixtures in a horizontal tube. Energy Power Eng. China 4(4):527534. 


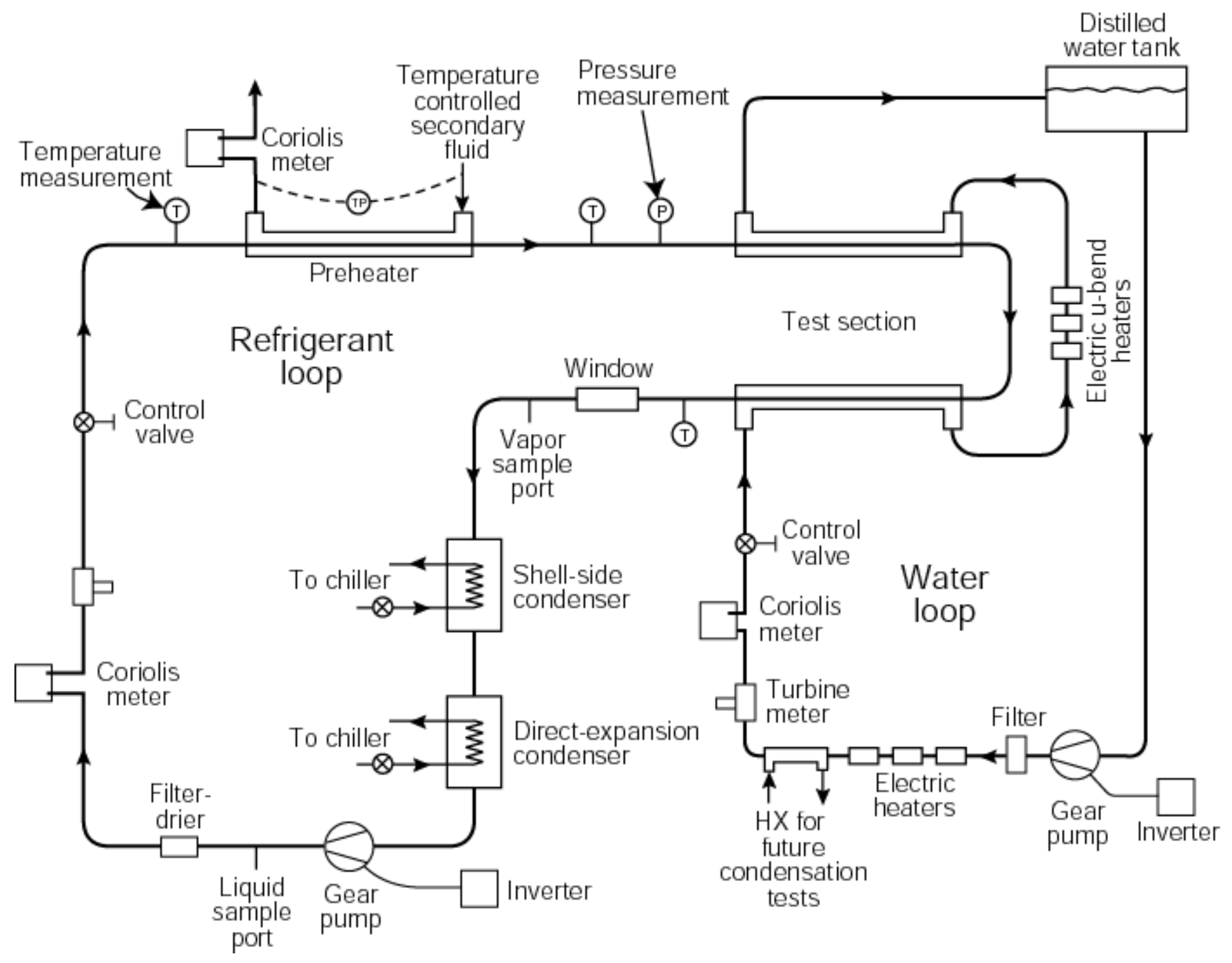

Figure 1 Schematic of test rig 


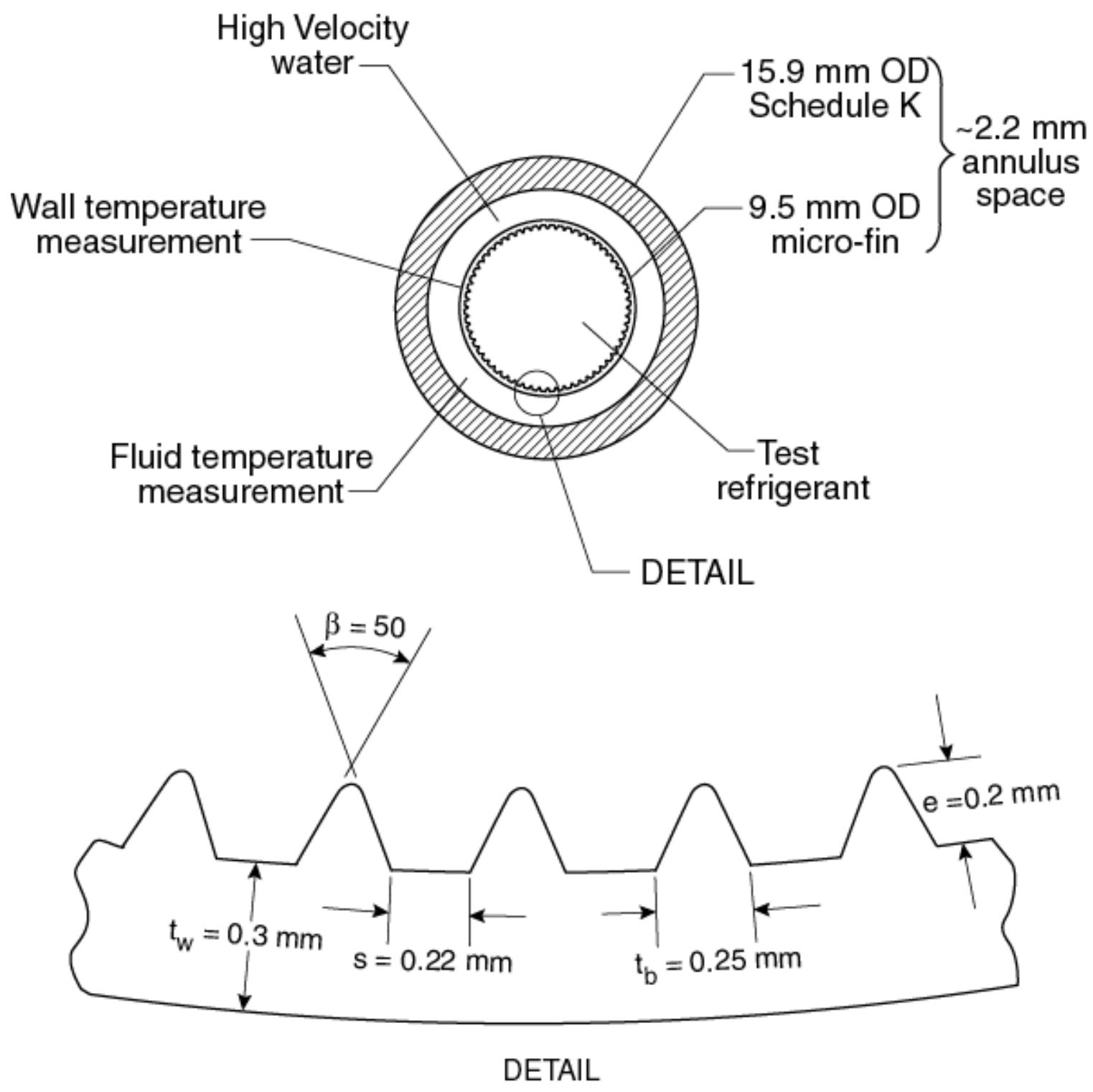

Figure 2 Test section cross section 


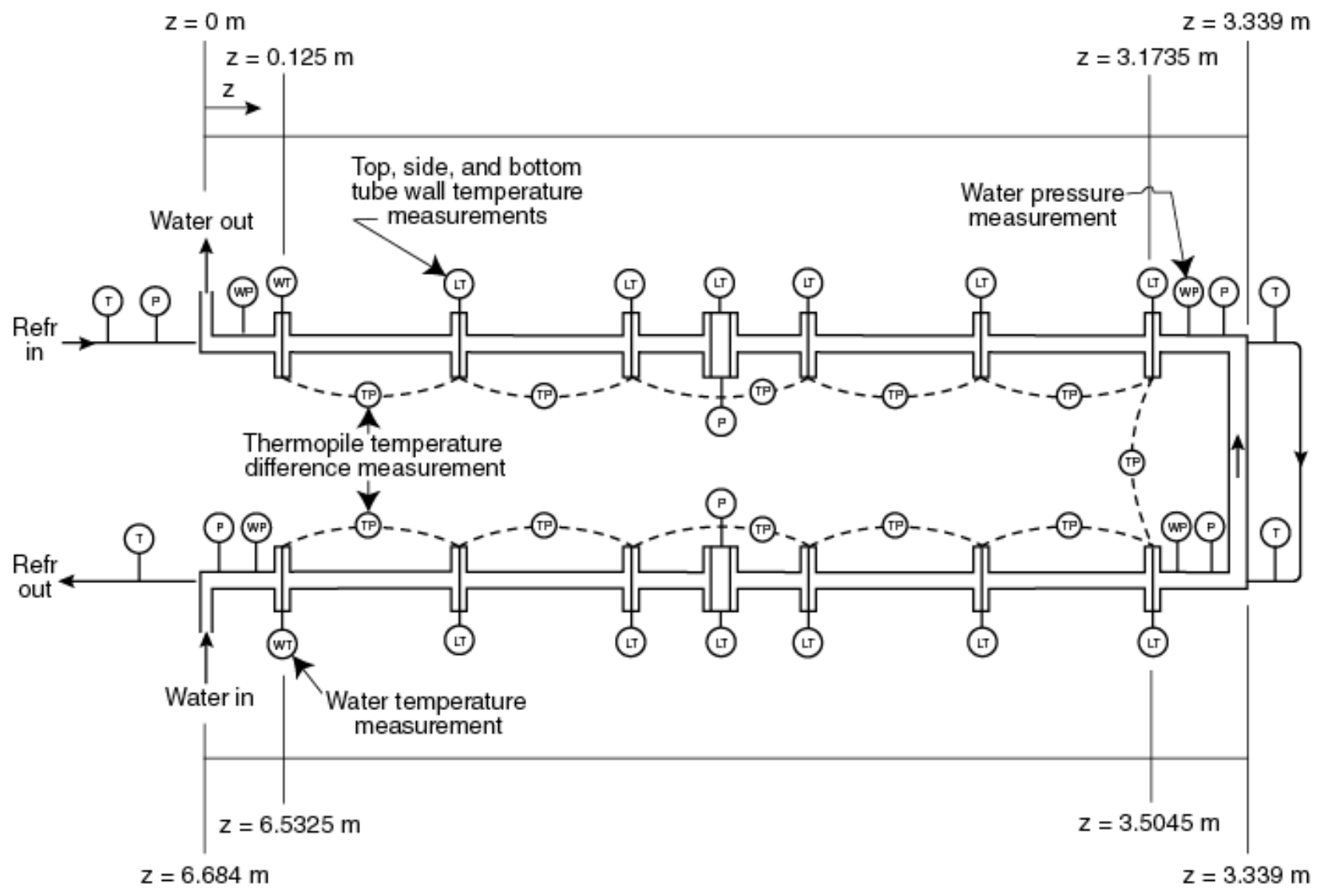

Figure 3 Detailed schematic of test section 


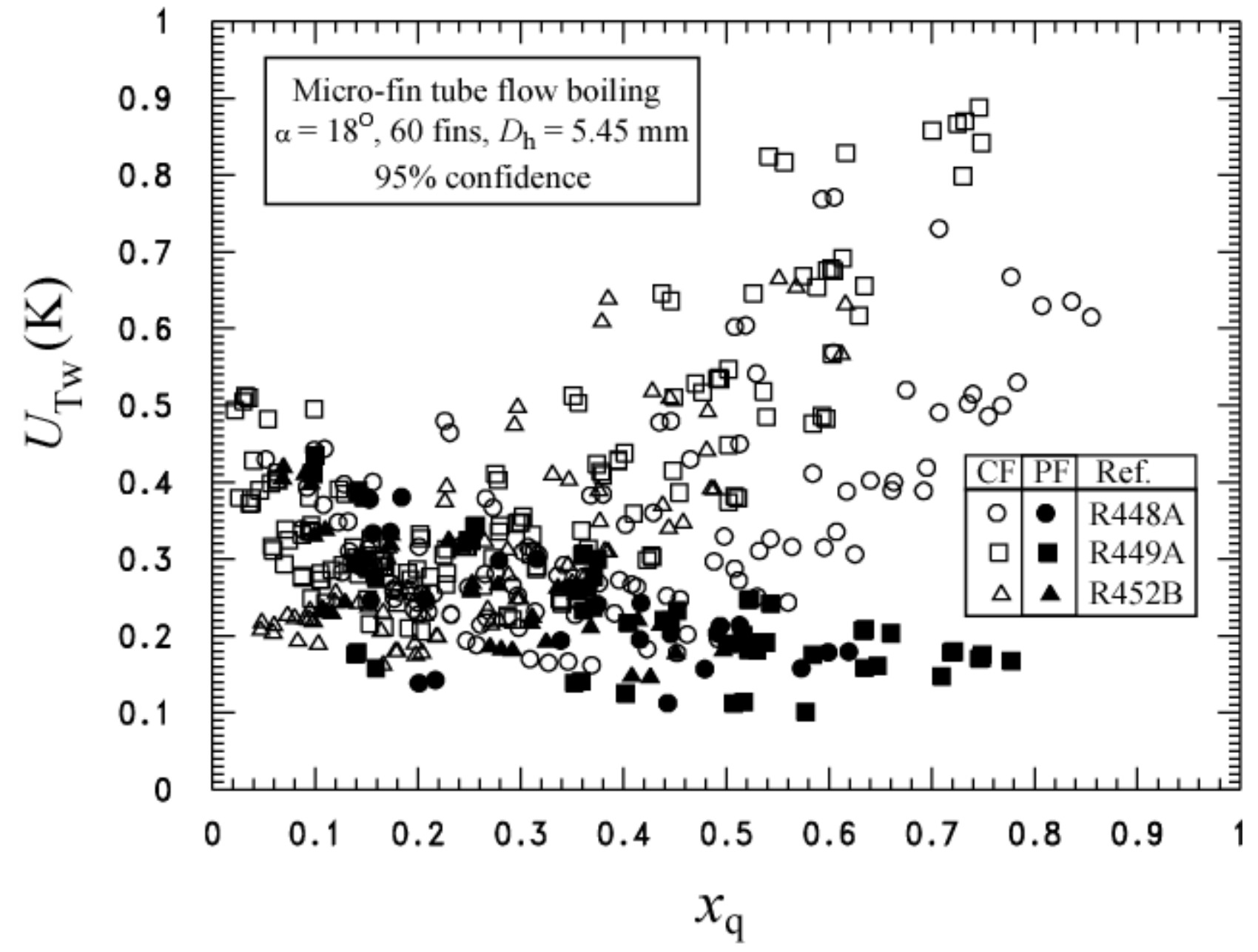

Figure 4 Relative uncertainty of inner wall temperature 


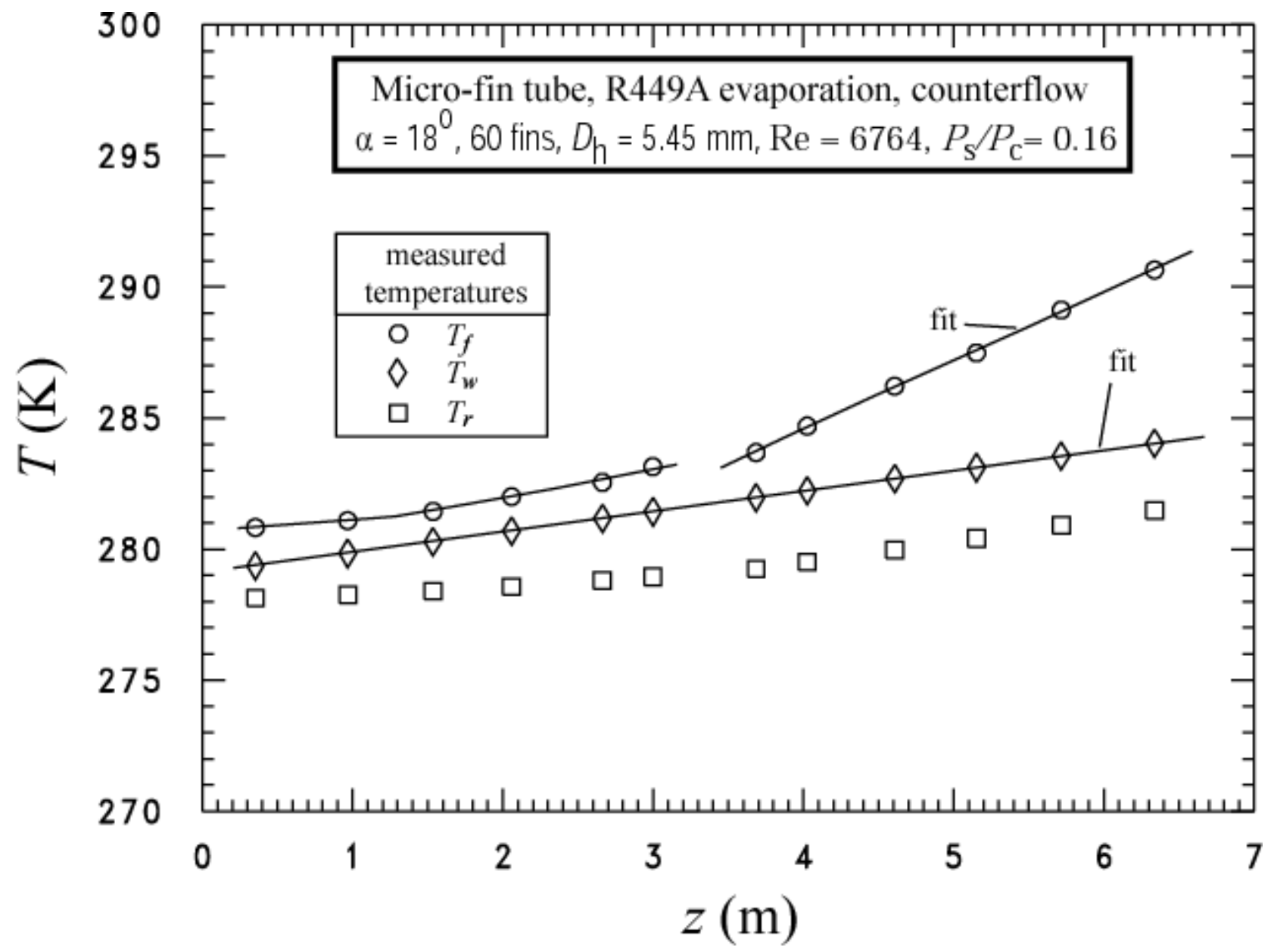

Figure 5 Counterflow temperature profiles for a 449A test 


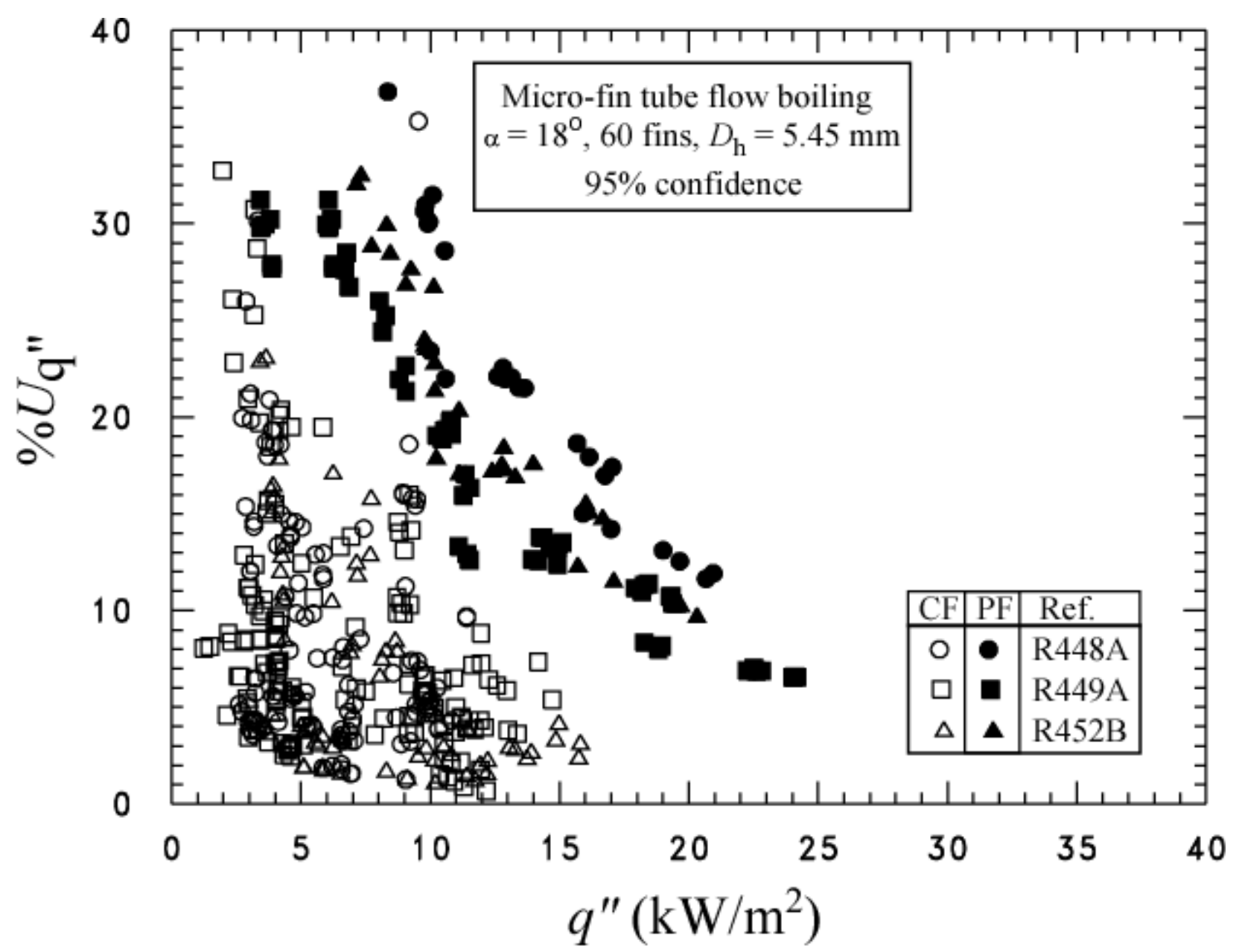

Figure 6 Relative uncertainty of water temperature gradient with respect to quality 


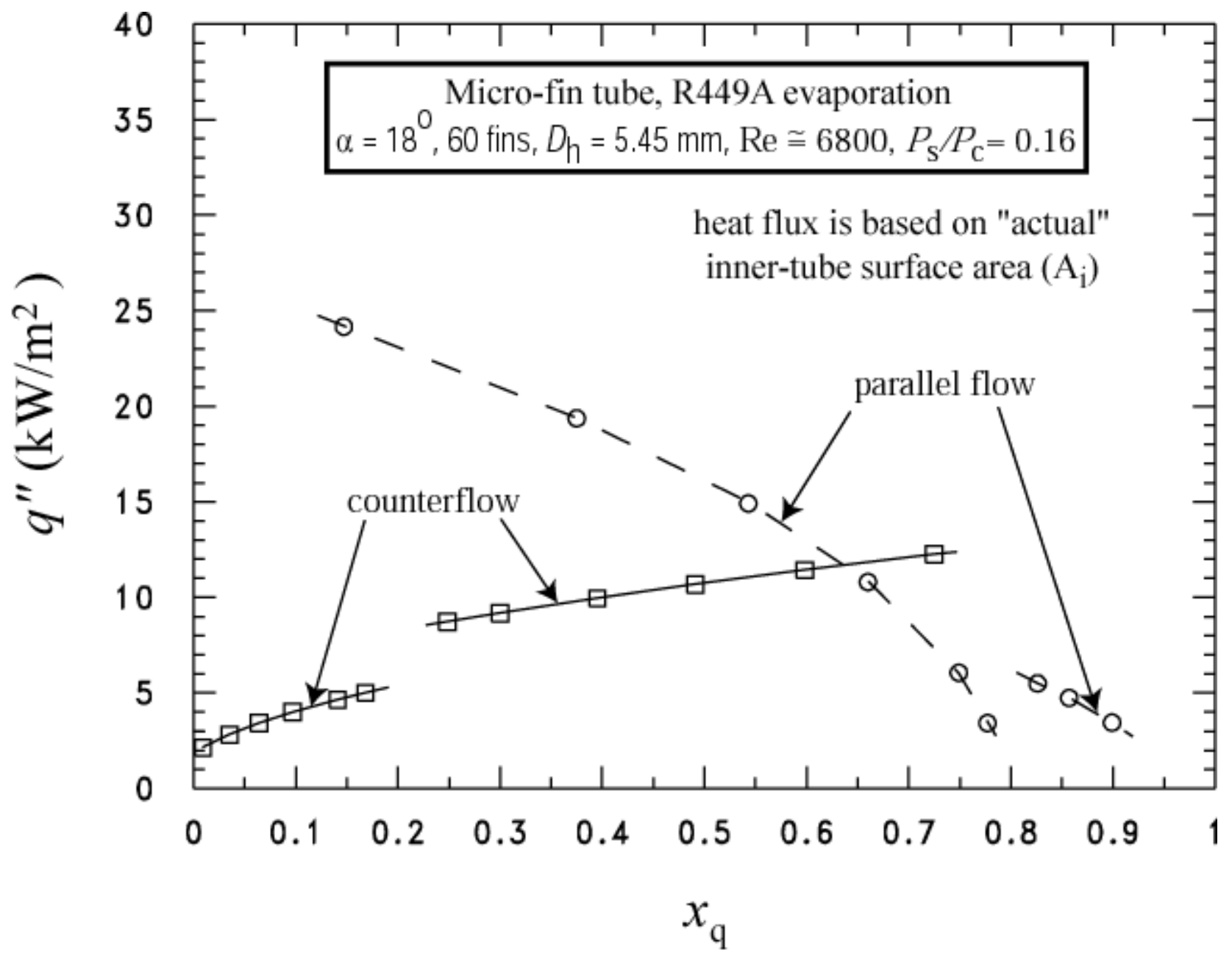

Figure 7 Heat Flux distribution for R449A 


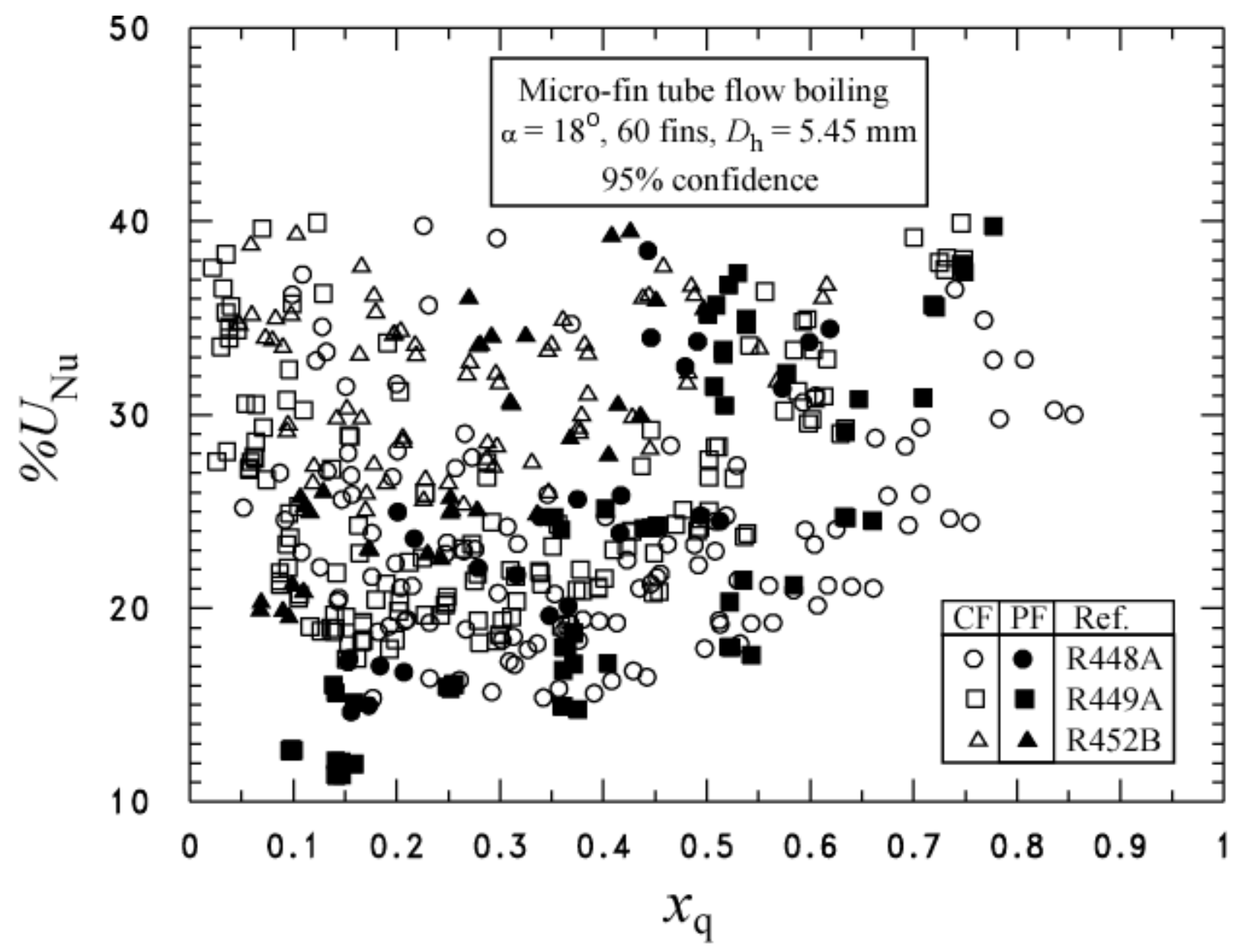

Figure 8 Relative uncertainty of the Nusselt number with respect to the quality 


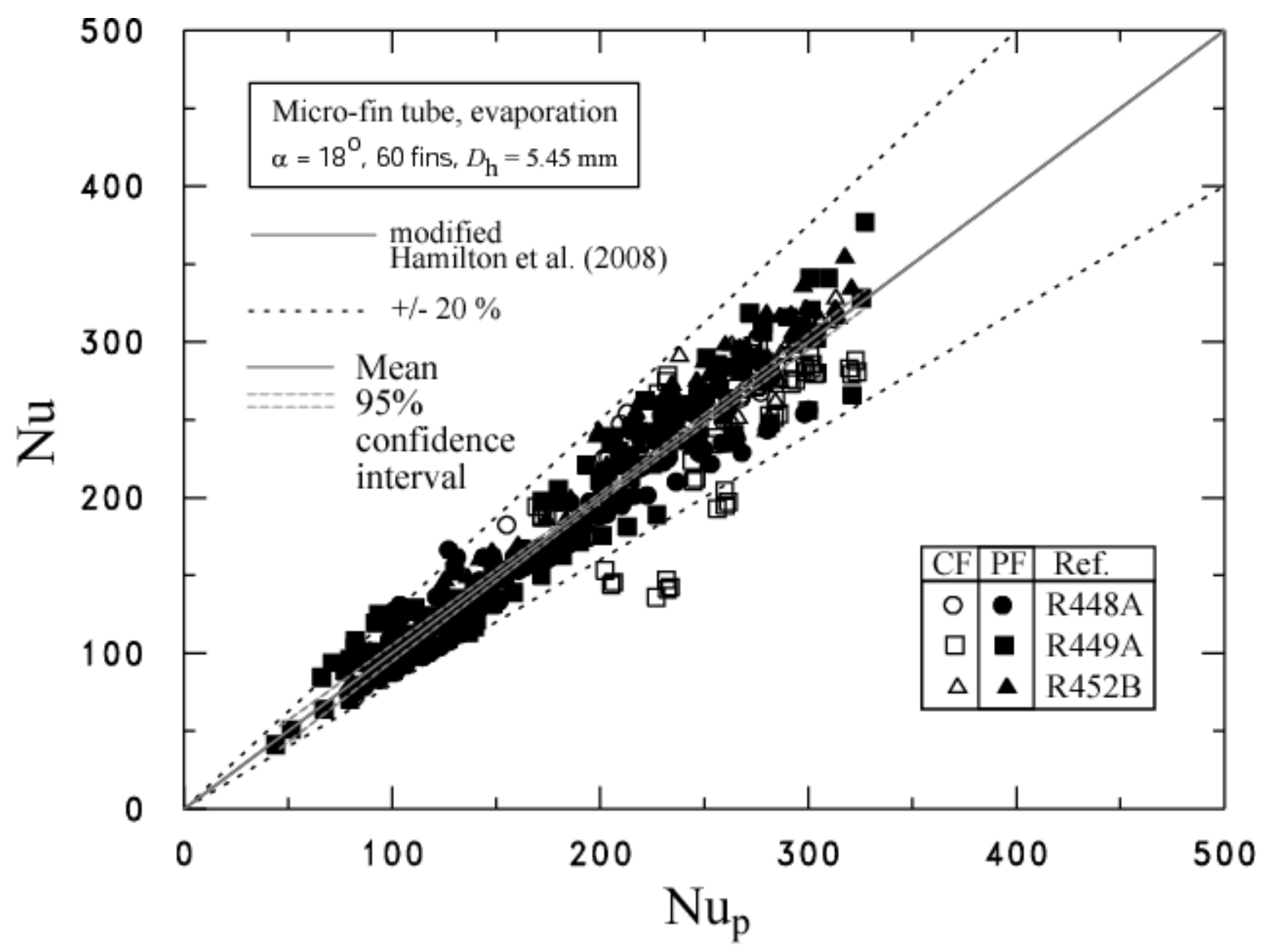

Figure 9 Comparison between measured Nusselt numbers and those predicted by the modified Hamilton et al. (2008) correlation 


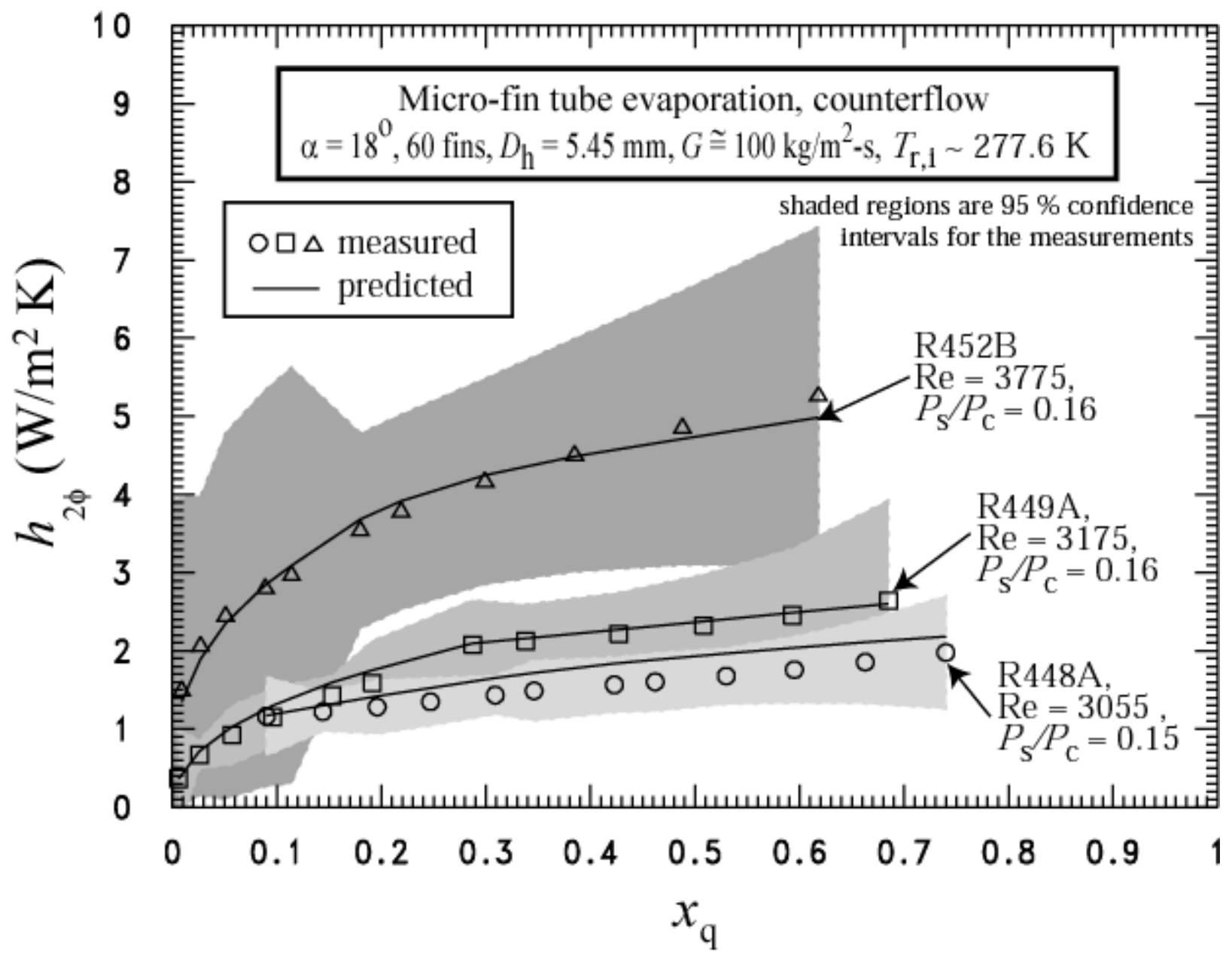

Figure 10 Flow boiling heat transfer coefficient for micro-fin tube versus thermodynamic quality for R452B, R449A, and R448A (counterflow) 


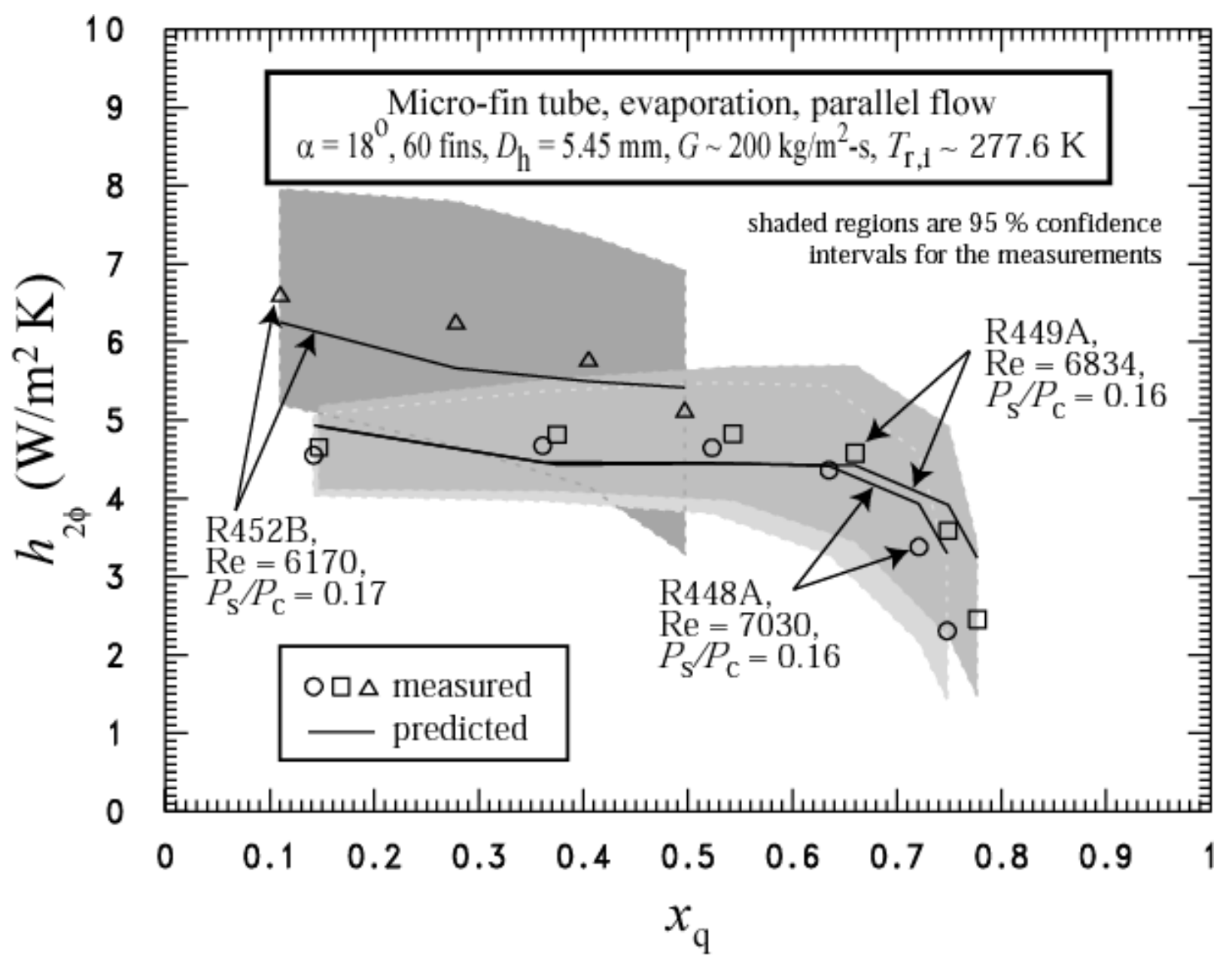

Figure 11 Flow boiling heat transfer coefficient for micro-fin tube versus thermodynamic quality for R452B, R449A, and R448A (parallel flow) 


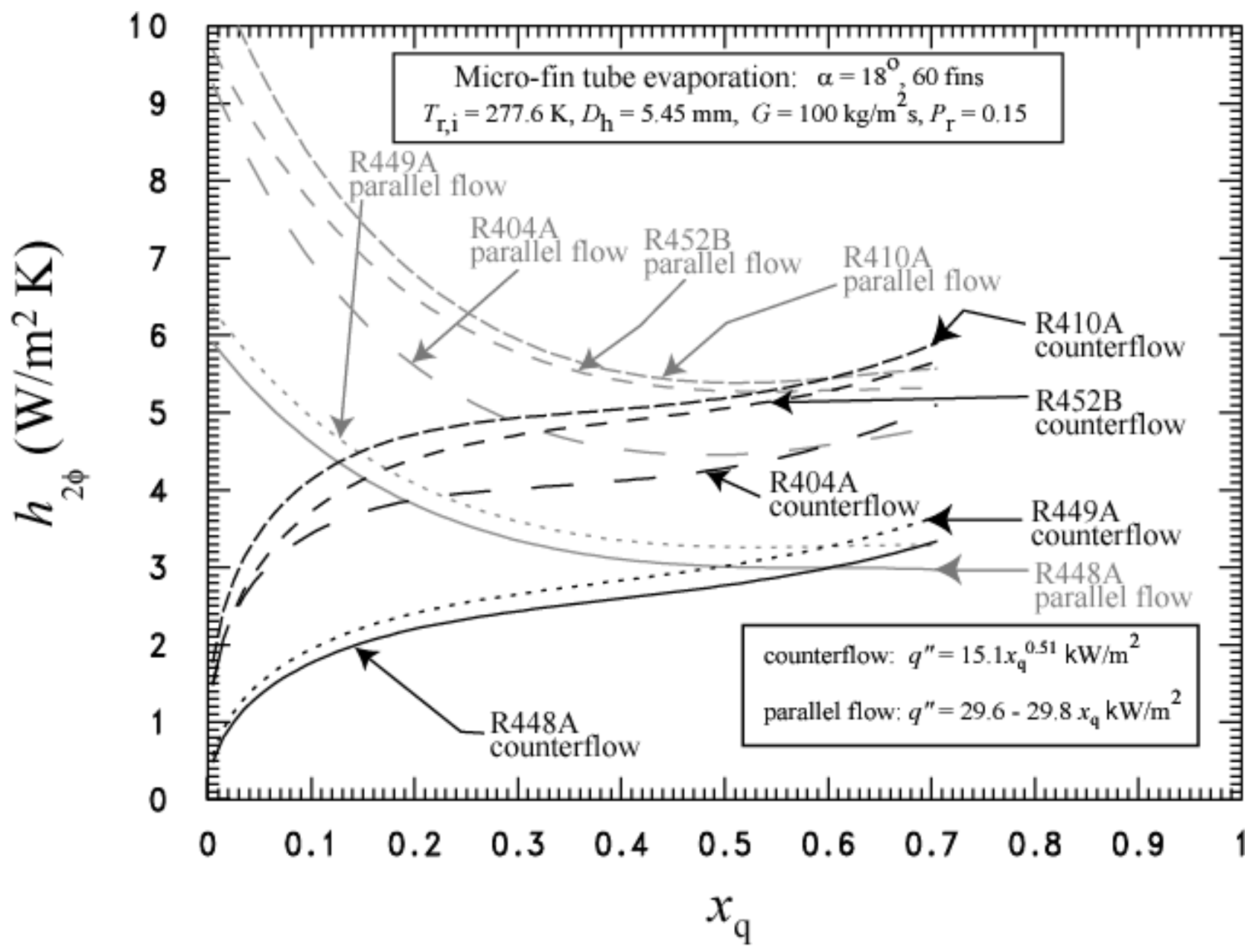

Figure 12 Flow boiling heat transfer coefficient versus thermodynamic quality for test refrigerants 


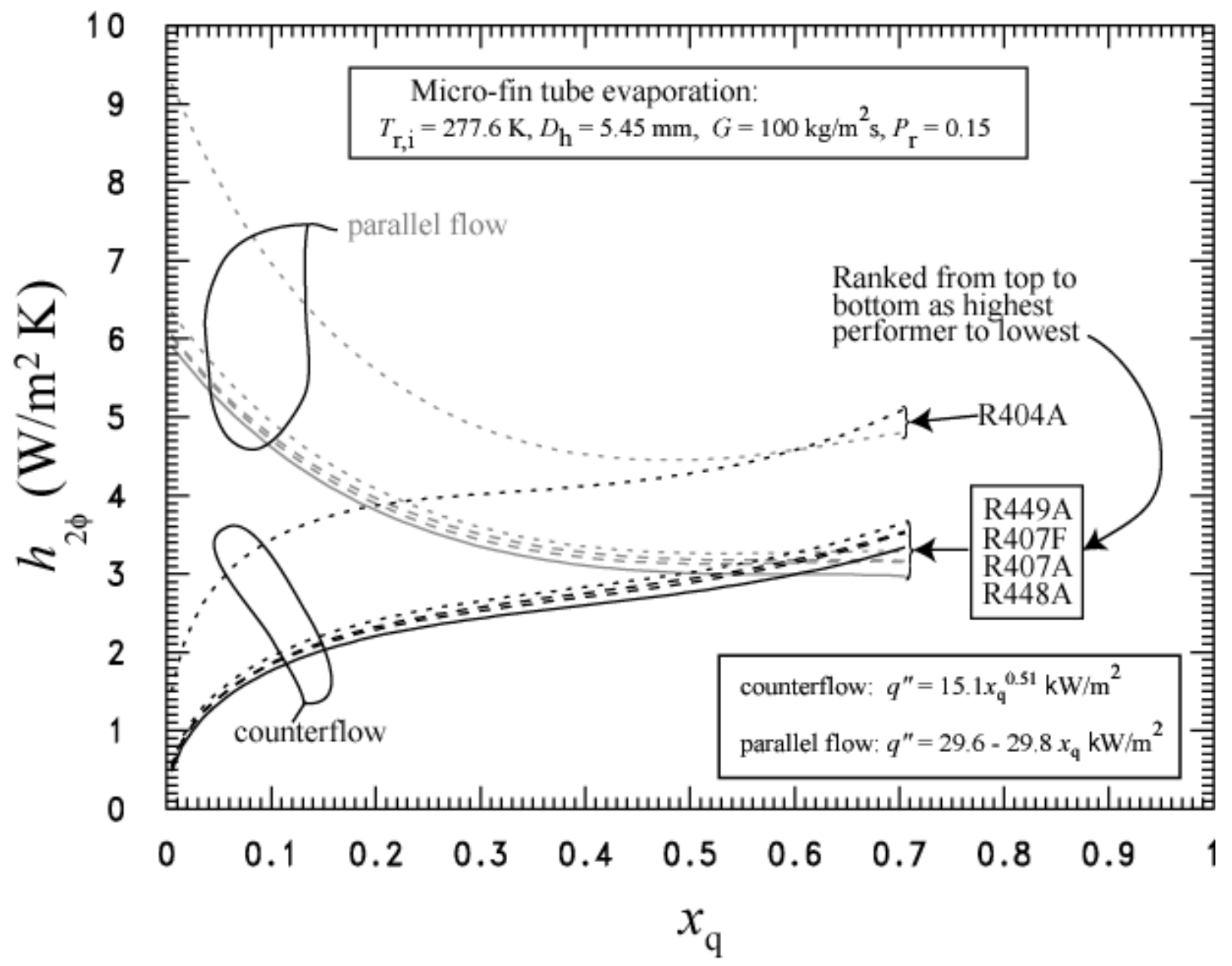

Figure 13 Flow boiling heat transfer coefficient versus thermodynamic quality for R404A replacements 
Table 1 Median estimated $95 \%$ relative expanded uncertainties for measurements $(U)$

\begin{tabular}{||c|c|c|c||}
\hline Parameter & Minimum & Maximum & $U \%$ \\
\hline$G_{\mathrm{r}}\left[\mathrm{kg} \mathrm{m}^{-2} \cdot \mathrm{s}^{-1}\right]$ & 91 & 304 & 2.0 \\
\hline$T_{\mathrm{s}}[\mathrm{K}]$ & 277.4 & 282.0 & $0.1(0.3 \mathrm{~K})$ \\
\hline$P[\mathrm{kPa}]$ & 692 & 917 & 1.5 \\
\hline$T_{\mathrm{w}}[\mathrm{K}]$ & 278.6 & 284.3 & $0.1(0.25 \mathrm{~K})$ \\
\hline$\dot{m}_{f}\left[\mathrm{~kg} \mathrm{~s}^{-1}\right]$ & 0.006 & 0.018 & 2.0 \\
\hline$T_{\mathrm{f}}[\mathrm{K}]$ & 281.0 & 321.0 & 0.1 \\
\hline$P_{\mathrm{f}}[\mathrm{kPa}]$ & 200 & 110 & 1.0 \\
\hline$q^{\prime \prime}\left[\mathrm{kW} \mathrm{m}{ }^{-2}\right]$ & 1.2 & 24.2 & 15 \\
\hline$\left(T_{\mathrm{d}}-T_{\mathrm{b}}\right) / T_{\mathrm{b}}$ & 0.004 & 0.02 & 3.0 \\
\hline $\mathrm{Nu}$ & 41 & 376 & 20 \\
\hline $\mathrm{Re}$ & 2759 & 11311 & 4.0 \\
\hline $\mathrm{Bo}$ & 0.000043 & 0.00062 & 16.0 \\
\hline $\mathrm{Pr}$ & 2.0 & 3.5 & 2.0 \\
\hline$P_{\mathrm{s}} / P_{\mathrm{c}}$ & 0.15 & 0.17 & 2.0 \\
\hline$x_{\mathrm{q}}$ & 0.02 & 0.86 & $0.44 \mathrm{~K}$ \\
\hline$\Delta T_{\mathrm{s}}[\mathrm{K}]$ & 1.00 & 5.28 & \\
\hline & & & \\
\hline \hline
\end{tabular}


Table 2 Representative properties from REFPROP (Lemmon et al. 2013)

\begin{tabular}{||c|c|c|c|c|c|c|c||}
\hline \hline Refrigerant & \multicolumn{2}{|c|}{$\begin{array}{c}\text { Evaluated at average test } \\
\text { conditions for each fluid }\end{array}$} & \multicolumn{5}{|c||}{ Evaluated at $T_{\mathrm{s}}=277.6 \mathrm{~K}$} \\
\cline { 2 - 8 } & $\begin{array}{c}T_{\mathrm{d}}-T_{\mathrm{b}} \\
(\mathrm{K})\end{array}$ & $\begin{array}{c}k_{1} \\
\left(\mathrm{~W} \mathrm{~m}^{-1} \mathrm{~K}^{-1}\right)\end{array}$ & $\operatorname{Pr}$ & $\begin{array}{c}\sigma \\
\left(\mathrm{mN} \mathrm{m}^{-1}\right)\end{array}$ & $\begin{array}{c}\rho_{\mathrm{I}} \\
(\mathrm{kg} \mathrm{m}-3)\end{array}$ & $\begin{array}{c}\rho_{\mathrm{v}} \\
(\mathrm{kg} \mathrm{m}-3)\end{array}$ & $\begin{array}{c}{\left[P_{\mathrm{s}}\right]_{x \mathrm{x}=0}} \\
(\mathrm{kPa})\end{array}$ \\
\hline R404A & 0.50 & 0.074 & 3.5 & 7.05 & 1132.8 & 35.8 & 700.6 \\
\hline R410A & 0.11 & 0.100 & 2.3 & 8.38 & 1151.9 & 34.6 & 920.5 \\
\hline R448A & 5.74 & 0.088 & 2.9 & 9.73 & 1180.7 & 28.8 & 717.1 \\
\hline R449A & 5.39 & 0.090 & 2.9 & 9.53 & 1180.2 & 26.0 & 708.2 \\
\hline R452B & 1.24 & 0.110 & 2.1 & 10.1 & 1072.5 & 28.1 & 889.7 \\
\hline
\end{tabular}




\section{APPENDIX A1}

Convective Boiling of R448A within a micro-fin tube (file: TaevapGWP3.dat)

\begin{tabular}{|c|c|c|c|c|c|c|c|c|c|c|}
\hline $\mathrm{Nu}$ & $\mathrm{Re}$ & $x_{\mathrm{q}}$ & Bo & $P_{\mathrm{s}} / P_{\mathrm{c}}$ & $T_{\mathrm{s}} / T_{\mathrm{c}}$ & $M_{\mathrm{w}}$ & $S v$ & $\operatorname{Pr}$ & flow & $U_{\mathrm{Nu}}$ \\
\hline 182.3 & 6559. & 0.191 & $0.71720 \times 10-4$ & 0.151 & 0.779 & 86.28 & 4.63 & 2.89 & $\mathrm{C}$ & 66.01 \\
\hline 166.2 & 6553. & 0.226 & $0.87600 \times 10-4$ & 0.151 & 0.780 & 86.28 & 3.98 & 2.90 & $\mathrm{C}$ & 39.77 \\
\hline 161.5 & 6547. & 0.266 & $0.10246 \times 10-3$ & 0.150 & 0.780 & 86.28 & 3.44 & 2.91 & $\mathrm{C}$ & 29.03 \\
\hline 161.8 & 6541. & 0.307 & $0.11628 \times 10-3$ & 0.150 & 0.781 & 86.28 & 3.01 & 2.93 & $\mathrm{C}$ & 24.21 \\
\hline 165.8 & 6533. & 0.362 & $0.13239 \times 10-3$ & 0.150 & 0.782 & 86.28 & 2.58 & 2.94 & $\mathrm{C}$ & 18.83 \\
\hline 169.6 & 6528. & 0.396 & $0.14157 \times 10-3$ & 0.150 & 0.782 & 86.28 & 2.38 & 2.95 & $\mathrm{C}$ & 19.32 \\
\hline 241.1 & 6504. & 0.488 & $0.21677 \times 10-3$ & 0.149 & 0.783 & 86.28 & 1.95 & 2.97 & $\mathrm{C}$ & 23.28 \\
\hline 244.2 & 6496. & 0.543 & $0.22105 \times 10-3$ & 0.149 & 0.784 & 86.28 & 1.76 & 2.99 & $\mathrm{C}$ & 19.21 \\
\hline 252.7 & 6483. & 0.640 & $0.22762 \times 10-3$ & 0.149 & 0.786 & 86.28 & 1.50 & 3.02 & $\mathrm{C}$ & 21.11 \\
\hline 263.4 & 6469. & 0.735 & $0.23427 \times 10-3$ & 0.149 & 0.787 & 86.28 & 1.31 & 3.04 & $\mathrm{C}$ & 24.64 \\
\hline 278.5 & 6454. & 0.836 & $0.24114 \times 10-3$ & 0.148 & 0.788 & 86.28 & 1.16 & 3.07 & $\mathrm{C}$ & 30.24 \\
\hline 299.4 & 6436. & 0.952 & $0.24874 \times 10-3$ & 0.147 & 0.790 & 86.28 & 1.02 & 3.09 & $\mathrm{C}$ & 40.49 \\
\hline 166.7 & 6494. & 0.193 & $0.76150 \times 10-4$ & 0.151 & 0.780 & 86.28 & 4.58 & 2.89 & $\mathrm{C}$ & 57.56 \\
\hline 161.6 & 6488. & 0.231 & $0.93530 \times 10-4$ & 0.151 & 0.780 & 86.28 & 3.91 & 2.90 & $\mathrm{C}$ & 35.66 \\
\hline 162.5 & 6482. & 0.273 & $0.10980 \times 10-3$ & 0.151 & 0.781 & 86.28 & 3.36 & 2.91 & $\mathrm{C}$ & 27.79 \\
\hline 166.7 & 6475. & 0.317 & $0.12495 \times 10-3$ & 0.151 & 0.781 & 86.28 & 2.92 & 2.93 & $\mathrm{C}$ & 23.32 \\
\hline 174.3 & 6467. & 0.376 & $0.14261 \times 10-3$ & 0.151 & 0.782 & 86.28 & 2.49 & 2.94 & $\mathrm{C}$ & 18.33 \\
\hline 180.1 & 6462. & 0.413 & $0.15271 \times 10-3$ & 0.151 & 0.783 & 86.28 & 2.28 & 2.95 & $\mathrm{C}$ & 19.24 \\
\hline 241.3 & 6436. & 0.508 & $0.21800 \times 10-3$ & 0.150 & 0.784 & 86.28 & 1.87 & 2.98 & $\mathrm{C}$ & 22.95 \\
\hline 244.4 & 6428. & 0.564 & $0.22141 \times 10-3$ & 0.149 & 0.785 & 86.28 & 1.70 & 2.99 & $\mathrm{C}$ & 19.25 \\
\hline 252.3 & 6414. & 0.661 & $0.22641 \times 10-3$ & 0.149 & 0.786 & 86.28 & 1.46 & 3.02 & $\mathrm{C}$ & 21.03 \\
\hline 262.9 & 6401. & 0.755 & $0.23156 \times 10-3$ & 0.149 & 0.787 & 86.28 & 1.28 & 3.05 & $\mathrm{C}$ & 24.44 \\
\hline 276.9 & 6386. & 0.855 & $0.23686 \times 10-3$ & 0.148 & 0.789 & 86.28 & 1.14 & 3.07 & $\mathrm{C}$ & 30.02 \\
\hline 296.7 & 6369. & 0.969 & $0.24267 \times 10-3$ & 0.148 & 0.791 & 86.28 & 1.01 & 3.09 & $\mathrm{C}$ & 40.10 \\
\hline 111.0 & 9024. & 0.110 & $0.35160 \times 10-4$ & 0.153 & 0.780 & 86.28 & 7.39 & 2.87 & $\mathrm{C}$ & 71.62 \\
\hline 123.7 & 9018. & 0.128 & $0.48390 \times 10-4$ & 0.153 & 0.780 & 86.28 & 6.50 & 2.87 & $\mathrm{C}$ & 34.55 \\
\hline 134.3 & 9012. & 0.151 & $0.60690 \times 10-4$ & 0.153 & 0.780 & 86.28 & 5.68 & 2.88 & $\mathrm{C}$ & 31.46 \\
\hline 143.8 & 9006. & 0.176 & $0.72080 \times 10-4$ & 0.153 & 0.781 & 86.28 & 4.97 & 2.88 & $\mathrm{C}$ & 23.89 \\
\hline 155.2 & 8997. & 0.210 & $0.85310 \times 10-4$ & 0.153 & 0.781 & 86.28 & 4.24 & 2.89 & $\mathrm{C}$ & 19.42 \\
\hline 162.0 & 8992. & 0.232 & $0.92750 \times 10-4$ & 0.153 & 0.781 & 86.28 & 3.89 & 2.90 & $\mathrm{C}$ & 19.25 \\
\hline 253.2 & 8959. & 0.297 & $0.15775 \times 10-3$ & 0.152 & 0.782 & 86.28 & 3.11 & 2.92 & $\mathrm{C}$ & 39.13 \\
\hline 248.6 & 8949. & 0.336 & $0.15698 \times 10-3$ & 0.152 & 0.782 & 86.28 & 2.77 & 2.93 & $\mathrm{C}$ & 18.17 \\
\hline 241.2 & 8931. & 0.402 & $0.15529 \times 10-3$ & 0.151 & 0.783 & 86.28 & 2.34 & 2.95 & $\mathrm{C}$ & 24.72 \\
\hline 235.1 & 8910. & 0.465 & $0.15387 \times 10-3$ & 0.151 & 0.784 & 86.28 & 2.04 & 2.97 & $\mathrm{C}$ & 28.41 \\
\hline 228.8 & 8887. & 0.529 & $0.15230 \times 10-3$ & 0.150 & 0.785 & 86.28 & 1.80 & 2.98 & $\mathrm{C}$ & 27.38 \\
\hline 222.5 & 8857. & 0.600 & $0.15050 \times 10-3$ & 0.149 & 0.785 & 86.28 & 1.60 & 3.00 & $\mathrm{C}$ & 58.36 \\
\hline 98.7 & 4966. & 0.117 & $0.79820 \times 10-4$ & 0.153 & 0.780 & 86.28 & 7.04 & 2.87 & $\mathrm{C}$ & 56.80 \\
\hline 108.4 & 4961. & 0.156 & $0.99860 \times 10-4$ & 0.153 & 0.780 & 86.28 & 5.50 & 2.88 & $\mathrm{C}$ & 26.86 \\
\hline 117.9 & 4956. & 0.201 & $0.11863 \times 10-3$ & 0.153 & 0.781 & 86.28 & 4.41 & 2.89 & $\mathrm{C}$ & 28.13 \\
\hline 127.6 & 4951. & 0.249 & $0.13611 \times 10-3$ & 0.153 & 0.782 & 86.28 & 3.64 & 2.90 & $\mathrm{C}$ & 23.39 \\
\hline 140.7 & 4945. & 0.314 & $0.15653 \times 10-3$ & 0.153 & 0.783 & 86.28 & 2.95 & 2.92 & $\mathrm{C}$ & 17.08 \\
\hline
\end{tabular}




\begin{tabular}{|c|c|c|c|c|c|c|c|c|c|c|}
\hline 148.8 & 4941. & 0.353 & $0.16822 \times 10-3$ & 0.153 & 0.783 & 86.28 & 2.64 & 2.93 & $\mathrm{C}$ & 20.74 \\
\hline 196.6 & 4925. & 0.455 & $0.22597 \times 10-3$ & 0.152 & 0.785 & 86.28 & 2.08 & 2.96 & $\mathrm{C}$ & 21.78 \\
\hline 196.7 & 4920. & 0.512 & $0.22324 \times 10-3$ & 0.152 & 0.785 & 86.28 & 1.86 & 2.97 & $\mathrm{C}$ & 19.37 \\
\hline 197.4 & 4911. & 0.607 & $0.21742 \times 10-3$ & 0.152 & 0.787 & 86.28 & 1.58 & 3.00 & $\mathrm{C}$ & 20.14 \\
\hline 198.8 & 4902. & 0.695 & $0.21226 \times 10-3$ & 0.152 & 0.788 & 86.28 & 1.39 & 3.02 & $\mathrm{C}$ & 24.29 \\
\hline 201.1 & 4894. & 0.783 & $0.20666 \times 10-3$ & 0.151 & 0.790 & 86.28 & 1.23 & 3.04 & $\mathrm{C}$ & 29.80 \\
\hline 204.3 & 4884. & 0.879 & $0.20027 \times 10-3$ & 0.151 & 0.791 & 86.28 & 1.10 & 3.07 & $\mathrm{C}$ & 40.43 \\
\hline 70.4 & 3074 & 0.090 & $0.11761 \times 10-3$ & 0.153 & 0.779 & 86.28 & 8.67 & 2.86 & $\mathrm{C}$ & 43.45 \\
\hline 74.2 & 3071. & 0.144 & $0.12486 \times 10-3$ & 0.153 & 0.780 & 86.28 & 5.92 & 2.87 & $\mathrm{C}$ & 20.53 \\
\hline 78.4 & 3067. & 0.196 & $0.13177 \times 10-3$ & 0.153 & 0.781 & 86.28 & 4.52 & 2.89 & $\mathrm{C}$ & 26.77 \\
\hline 82.7 & 3064. & 0.247 & $0.13823 \times 10-3$ & 0.153 & 0.782 & 86.28 & 3.67 & 2.90 & $\mathrm{C}$ & 22.81 \\
\hline 88.5 & 3060. & 0.309 & $0.14573 \times 10-3$ & 0.153 & 0.783 & 86.28 & 2.98 & 2.92 & $\mathrm{C}$ & 17.25 \\
\hline 92.2 & 3058. & 0.346 & $0.15015 \times 10-3$ & 0.153 & 0.783 & 86.28 & 2.69 & 2.93 & $\mathrm{C}$ & 25.88 \\
\hline 97.6 & 3052. & 0.423 & $0.15573 \times 10-3$ & 0.152 & 0.784 & 86.28 & 2.23 & 2.95 & $\mathrm{C}$ & 22.49 \\
\hline 100.3 & 3050 & 0.462 & $0.15719 \times 10-3$ & 0.152 & 0.785 & 86.28 & 2.05 & 2.96 & $\mathrm{C}$ & 23.31 \\
\hline 105.6 & 3046. & 0.530 & $0.15911 \times 10-3$ & 0.152 & 0.786 & 86.28 & 1.80 & 2.98 & $\mathrm{C}$ & 21.44 \\
\hline 111.3 & 3043. & 0.595 & $0.16114 \times 10-3$ & 0.152 & 0.787 & 86.28 & 1.61 & 2.99 & $\mathrm{C}$ & 24.05 \\
\hline 118.1 & 3039. & 0.663 & $0.16321 \times 10-3$ & 0.152 & 0.788 & 86.28 & 1.45 & 3.01 & $\mathrm{C}$ & 28.79 \\
\hline 126.8 & 3035. & 0.740 & $0.16548 \times 10-3$ & 0.152 & 0.789 & 86.28 & 1.31 & 3.03 & $\mathrm{C}$ & 36.49 \\
\hline 248.9 & 6464. & 0.156 & $0.47149 \times 10-3$ & 0.154 & 0.781 & 86.28 & 5.52 & 2.87 & $\mathrm{P}$ & 14.64 \\
\hline 255.7 & 6438. & 0.348 & $0.38753 \times 10-3$ & 0.154 & 0.784 & 86.28 & 2.68 & 2.93 & $\mathrm{P}$ & 19.61 \\
\hline 254.8 & 6420. & 0.494 & $0.30829 \times 10-3$ & 0.154 & 0.786 & 86.28 & 1.92 & 2.97 & $\mathrm{P}$ & 24.78 \\
\hline 240.1 & 6407. & 0.599 & $0.23252 \times 10-3$ & 0.153 & 0.788 & 86.28 & 1.60 & 2.99 & $\mathrm{P}$ & 33.76 \\
\hline 191.1 & 6395. & 0.684 & $0.14206 \times 10-3$ & 0.153 & 0.789 & 86.28 & 1.41 & 3.01 & $\mathrm{P}$ & 50.21 \\
\hline 141.4 & 6390. & 0.713 & $0.91330 \times 10-4$ & 0.153 & 0.789 & 86.28 & 1.35 & 3.02 & $\mathrm{P}$ & 51.09 \\
\hline 266.6 & 6350. & 0.770 & $0.14072 \times 10-3$ & 0.151 & 0.789 & 86.28 & 1.26 & 3.04 & $\mathrm{P}$ & 79.16 \\
\hline 275.6 & 6343. & 0.804 & $0.12116 \times 10-3$ & 0.150 & 0.789 & 86.28 & 1.20 & 3.05 & $\mathrm{P}$ & 52.93 \\
\hline 278.0 & 6332. & 0.850 & $0.87270 \times 10-4$ & 0.150 & 0.790 & 86.28 & 1.14 & 3.06 & $\mathrm{P}$ & 135.12 \\
\hline 245.6 & 6322. & 0.880 & $0.55450 \times 10-4$ & 0.149 & 0.790 & 86.28 & 1.10 & 3.07 & $\mathrm{P}$ & 128.20 \\
\hline 136.3 & 6313. & 0.896 & $0.22240 \times 10-4$ & 0.149 & 0.790 & 86.28 & 1.08 & 3.07 & $\mathrm{P}$ & 542.06 \\
\hline 284.2 & 8955. & 0.184 & $0.32318 \times 10-3$ & 0.153 & 0.781 & 86.28 & 4.77 & 2.89 & $\mathrm{P}$ & 17.01 \\
\hline 287.6 & 8927. & 0.316 & $0.26797 \times 10-3$ & 0.152 & 0.782 & 86.28 & 2.93 & 2.92 & $\mathrm{P}$ & 21.72 \\
\hline 283.1 & 8904. & 0.417 & $0.21620 \times 10-3$ & 0.152 & 0.784 & 86.28 & 2.26 & 2.95 & $\mathrm{P}$ & 25.83 \\
\hline 266.3 & 8886. & 0.491 & $0.16726 \times 10-3$ & 0.152 & 0.785 & 86.28 & 1.93 & 2.97 & $\mathrm{P}$ & 33.79 \\
\hline 220.0 & 8867. & 0.554 & $0.10934 \times 10-3$ & 0.151 & 0.786 & 86.28 & 1.73 & 2.99 & $\mathrm{P}$ & 47.90 \\
\hline 176.2 & 8858. & 0.577 & $0.77000 \times 10-4$ & 0.151 & 0.786 & 86.28 & 1.66 & 2.99 & $\mathrm{P}$ & 48.88 \\
\hline 319.7 & 8781. & 0.629 & $0.12261 \times 10-3$ & 0.148 & 0.785 & 86.28 & 1.53 & 3.02 & $\mathrm{P}$ & 65.68 \\
\hline 329.3 & 8768. & 0.658 & $0.10744 \times 10-3$ & 0.148 & 0.785 & 86.28 & 1.46 & 3.02 & $\mathrm{P}$ & 44.86 \\
\hline 336.4 & 8747. & 0.700 & $0.81270 \times 10-4$ & 0.147 & 0.785 & 86.28 & 1.38 & 3.04 & $\mathrm{P}$ & 99.15 \\
\hline 318.9 & 8726. & 0.728 & $0.56710 \times 10-4$ & 0.146 & 0.786 & 86.28 & 1.33 & 3.04 & $\mathrm{P}$ & 96.78 \\
\hline 244.4 & 8706. & 0.747 & $0.31090 \times 10-4$ & 0.146 & 0.785 & 86.28 & 1.29 & 3.05 & $\mathrm{P}$ & 823.88 \\
\hline 30.8 & 8683. & 0.756 & $0.278 \times 10-5$ & 0.145 & 0.785 & 86.28 & 1.28 & 3.06 & $\mathrm{P}$ & 831.77 \\
\hline 234.1 & 4899. & 0.207 & $0.51156 \times 10-3$ & 0.153 & 0.781 & 86.28 & 4.30 & 2.89 & $\mathrm{P}$ & 16.69 \\
\hline 247.2 & 4879. & 0.416 & $0.41730 \times 10-3$ & 0.153 & 0.784 & 86.28 & 2.26 & 2.95 & $\mathrm{P}$ & 23.88 \\
\hline 254.1 & 4867. & 0.573 & $0.32809 \times 10-3$ & 0.153 & 0.787 & 86.28 & 1.67 & 2.99 & $\mathrm{P}$ & 31.37 \\
\hline 246.5 & 4858. & 0.685 & $0.24251 \times 10-3$ & 0.153 & 0.789 & 86.28 & 1.41 & 3.01 & $\mathrm{P}$ & 43.23 \\
\hline 198.5 & 4851. & 0.772 & $0.14017 \times 10-3$ & 0.153 & 0.790 & 86.28 & 1.25 & 3.03 & $\mathrm{P}$ & 67.80 \\
\hline 139.7 & 4848. & 0.800 & $0.82740 \times 10-4$ & 0.153 & 0.791 & 86.28 & 1.21 & 3.04 & $\mathrm{P}$ & 69.31 \\
\hline 315.4 & 4830. & 0.852 & $0.13707 \times 10-3$ & 0.151 & 0.791 & 86.28 & 1.14 & 3.06 & $\mathrm{P}$ & 131.38 \\
\hline
\end{tabular}




\begin{tabular}{|c|c|c|c|c|c|c|c|c|c|c|}
\hline 351.0 & 4826. & 0.885 & $0.11630 \times 10-3$ & 0.151 & 0.791 & 86.28 & 1.10 & 3.06 & $\mathrm{P}$ & 78.83 \\
\hline 428.1 & 4821. & 0.929 & $0.80280 \times 10-4$ & 0.151 & 0.792 & 86.28 & 1.05 & 3.08 & $\mathrm{P}$ & 242.82 \\
\hline 494.6 & 4816. & 0.954 & $0.46460 \times 10-4$ & 0.151 & 0.792 & 86.28 & 1.02 & 3.08 & $\mathrm{P}$ & 257.08 \\
\hline 311.3 & 4811. & 0.966 & $0.11200 \times 10-4$ & 0.150 & 0.792 & 86.28 & 1.01 & 3.09 & $\mathrm{P}$ & 654.12 \\
\hline 167.4 & 2801. & 0.217 & $0.55789 \times 10-3$ & 0.153 & 0.781 & 86.28 & 4.13 & 2.89 & $\mathrm{P}$ & 23.59 \\
\hline 182.3 & 2790. & 0.443 & $0.44744 \times 10-3$ & 0.153 & 0.785 & 86.28 & 2.13 & 2.95 & $\mathrm{P}$ & 38.48 \\
\hline 194.5 & 2783. & 0.610 & $0.34256 \times 10-3$ & 0.153 & 0.788 & 86.28 & 1.57 & 2.99 & $\mathrm{P}$ & 59.45 \\
\hline 195.1 & 2779. & 0.725 & $0.24170 \times 10-3$ & 0.153 & 0.790 & 86.28 & 1.33 & 3.02 & $\mathrm{P}$ & 77.14 \\
\hline 152.2 & 2777. & 0.808 & $0.12109 \times 10-3$ & 0.153 & 0.791 & 86.28 & 1.20 & 3.04 & $\mathrm{P}$ & 124.48 \\
\hline 85.0 & 2776. & 0.830 & $0.53400 \times 10-4$ & 0.153 & 0.791 & 86.28 & 1.17 & 3.05 & $\mathrm{P}$ & 127.13 \\
\hline 385.5 & 2772. & 0.873 & $0.13683 \times 10-3$ & 0.153 & 0.792 & 86.28 & 1.11 & 3.06 & $\mathrm{P}$ & 512.48 \\
\hline 578.7 & 2771. & 0.905 & $0.11126 \times 10-3$ & 0.153 & 0.792 & 86.28 & 1.07 & 3.07 & $\mathrm{P}$ & 166.76 \\
\hline 92.1 & 6513. & 0.020 & $0.63990 \times 10-4$ & 0.154 & 0.779 & 86.28 & 22.08 & 2.84 & $\mathrm{C}$ & 56.07 \\
\hline 101.5 & 6508. & 0.052 & $0.80110 \times 10-4$ & 0.154 & 0.780 & 86.28 & 13.00 & 2.85 & $\mathrm{C}$ & 25.19 \\
\hline 109.9 & 6502. & 0.087 & $0.95160 \times 10-4$ & 0.154 & 0.780 & 86.28 & 8.88 & 2.86 & $\mathrm{C}$ & 27.00 \\
\hline 117.9 & 6496. & 0.126 & $0.10914 \times 10-3$ & 0.154 & 0.781 & 86.28 & 6.62 & 2.87 & $\mathrm{C}$ & 22.13 \\
\hline 127.6 & 6488. & 0.177 & $0.12543 \times 10-3$ & 0.154 & 0.781 & 86.28 & 4.95 & 2.88 & $\mathrm{C}$ & 15.35 \\
\hline 133.6 & 6483. & 0.208 & $0.13469 \times 10-3$ & 0.154 & 0.782 & 86.28 & 4.28 & 2.89 & $\mathrm{C}$ & 19.33 \\
\hline 186.3 & 6460. & 0.292 & $0.19733 \times 10-3$ & 0.153 & 0.782 & 86.28 & 3.14 & 2.91 & $\mathrm{C}$ & 15.67 \\
\hline 188.9 & 6452. & 0.342 & $0.20099 \times 10-3$ & 0.153 & 0.783 & 86.28 & 2.72 & 2.93 & $\mathrm{C}$ & 15.37 \\
\hline 194.7 & 6438. & 0.429 & $0.20663 \times 10-3$ & 0.153 & 0.784 & 86.28 & 2.20 & 2.95 & $\mathrm{C}$ & 16.76 \\
\hline 201.4 & 6424. & 0.513 & $0.21234 \times 10-3$ & 0.152 & 0.786 & 86.28 & 1.85 & 2.97 & $\mathrm{C}$ & 19.17 \\
\hline 210.0 & 6408. & 0.604 & $0.21827 \times 10-3$ & 0.152 & 0.787 & 86.28 & 1.59 & 3.00 & $\mathrm{C}$ & 23.29 \\
\hline 221.5 & 6389. & 0.707 & $0.22485 \times 10-3$ & 0.151 & 0.788 & 86.28 & 1.36 & 3.03 & $\mathrm{C}$ & 29.35 \\
\hline 120.9 & 9181. & 0.089 & $0.36730 \times 10-4$ & 0.153 & 0.780 & 86.28 & 8.72 & 2.86 & $\mathrm{C}$ & 85.58 \\
\hline 130.8 & 9175. & 0.109 & $0.50120 \times 10-4$ & 0.153 & 0.780 & 86.28 & 7.47 & 2.87 & $\mathrm{C}$ & 37.26 \\
\hline 139.1 & 9168. & 0.132 & $0.62570 \times 10-4$ & 0.153 & 0.780 & 86.28 & 6.37 & 2.87 & $\mathrm{C}$ & 33.25 \\
\hline 146.6 & 9162. & 0.157 & $0.74100 \times 10-4$ & 0.153 & 0.780 & 86.28 & 5.47 & 2.88 & $\mathrm{C}$ & 25.87 \\
\hline 155.7 & 9152. & 0.193 & $0.87490 \times 10-4$ & 0.153 & 0.781 & 86.28 & 4.58 & 2.89 & $\mathrm{C}$ & 19.07 \\
\hline 160.9 & 9147. & 0.215 & $0.95030 \times 10-4$ & 0.153 & 0.781 & 86.28 & 4.16 & 2.89 & $\mathrm{C}$ & 21.14 \\
\hline 221.8 & 9112. & 0.276 & $0.14552 \times 10-3$ & 0.152 & 0.781 & 86.28 & 3.32 & 2.91 & $\mathrm{C}$ & 23.04 \\
\hline 224.9 & 9102. & 0.313 & $0.15118 \times 10-3$ & 0.152 & 0.782 & 86.28 & 2.96 & 2.92 & $\mathrm{C}$ & 18.53 \\
\hline 231.2 & 9083. & 0.380 & $0.16065 \times 10-3$ & 0.151 & 0.783 & 86.28 & 2.47 & 2.94 & $\mathrm{C}$ & 19.41 \\
\hline 237.7 & 9062. & 0.446 & $0.16980 \times 10-3$ & 0.151 & 0.784 & 86.28 & 2.12 & 2.96 & $\mathrm{C}$ & 21.26 \\
\hline 246.0 & 9036. & 0.519 & $0.17936 \times 10-3$ & 0.150 & 0.784 & 86.28 & 1.84 & 2.98 & $\mathrm{C}$ & 24.78 \\
\hline 256.3 & 9005. & 0.605 & $0.19001 \times 10-3$ & 0.149 & 0.785 & 86.28 & 1.59 & 3.00 & $\mathrm{C}$ & 30.97 \\
\hline 116.8 & 9198. & 0.079 & $0.36760 \times 10-4$ & 0.154 & 0.780 & 86.28 & 9.55 & 2.86 & $\mathrm{C}$ & 84.04 \\
\hline 127.3 & 9192. & 0.099 & $0.50040 \times 10-4$ & 0.153 & 0.780 & 86.28 & 8.07 & 2.86 & $\mathrm{C}$ & 36.21 \\
\hline 136.1 & 9185. & 0.122 & $0.62380 \times 10-4$ & 0.153 & 0.780 & 86.28 & 6.81 & 2.87 & $\mathrm{C}$ & 32.80 \\
\hline 143.8 & 9178. & 0.147 & $0.73810 \times 10-4$ & 0.153 & 0.780 & 86.28 & 5.79 & 2.88 & $\mathrm{C}$ & 25.62 \\
\hline 153.1 & 9169. & 0.182 & $0.87090 \times 10-4$ & 0.153 & 0.781 & 86.28 & 4.81 & 2.89 & $\mathrm{C}$ & 18.79 \\
\hline 158.5 & 9163. & 0.204 & $0.94560 \times 10-4$ & 0.153 & 0.781 & 86.28 & 4.35 & 2.89 & $\mathrm{C}$ & 21.05 \\
\hline 219.4 & 9128. & 0.265 & $0.14505 \times 10-3$ & 0.152 & 0.781 & 86.28 & 3.44 & 2.91 & $\mathrm{C}$ & 22.95 \\
\hline 222.5 & 9119. & 0.302 & $0.15071 \times 10-3$ & 0.152 & 0.782 & 86.28 & 3.05 & 2.92 & $\mathrm{C}$ & 18.35 \\
\hline 228.6 & 9099. & 0.368 & $0.16018 \times 10-3$ & 0.151 & 0.783 & 86.28 & 2.54 & 2.94 & $\mathrm{C}$ & 19.20 \\
\hline 235.4 & 9078. & 0.435 & $0.16931 \times 10-3$ & 0.151 & 0.783 & 86.28 & 2.17 & 2.96 & $\mathrm{C}$ & 21.03 \\
\hline 243.4 & 9052. & 0.508 & $0.17887 \times 10-3$ & 0.150 & 0.784 & 86.28 & 1.88 & 2.98 & $\mathrm{C}$ & 24.49 \\
\hline 253.8 & 9021. & 0.593 & $0.18951 \times 10-3$ & 0.149 & 0.785 & 86.28 & 1.62 & 3.00 & $\mathrm{C}$ & 30.64 \\
\hline 86.9 & 4992. & 0.054 & $0.77090 \times 10-4$ & 0.154 & 0.779 & 86.28 & 12.56 & 2.85 & $\mathrm{C}$ & 50.37 \\
\hline
\end{tabular}




\begin{tabular}{|c|c|c|c|c|c|c|c|c|c|c|}
\hline 93.7 & 4988. & 0.092 & $0.92940 \times 10-4$ & 0.154 & 0.780 & 86.28 & 8.55 & 2.86 & $\mathrm{C}$ & 24.57 \\
\hline 100.4 & 4983. & 0.133 & $0.10778 \times 10-3$ & 0.154 & 0.780 & 86.28 & 6.33 & 2.87 & $\mathrm{C}$ & 27.08 \\
\hline 107.2 & 4979. & 0.176 & $0.12159 \times 10-3$ & 0.154 & 0.781 & 86.28 & 4.97 & 2.88 & $\mathrm{C}$ & 21.62 \\
\hline 116.0 & 4973. & 0.232 & $0.13770 \times 10-3$ & 0.154 & 0.782 & 86.28 & 3.88 & 2.90 & $\mathrm{C}$ & 16.37 \\
\hline 121.5 & 4970. & 0.267 & $0.14690 \times 10-3$ & 0.154 & 0.782 & 86.28 & 3.42 & 2.90 & $\mathrm{C}$ & 18.91 \\
\hline 165.8 & 4955. & 0.357 & $0.20591 \times 10-3$ & 0.153 & 0.783 & 86.28 & 2.62 & 2.93 & $\mathrm{C}$ & 15.84 \\
\hline 168.3 & 4949. & 0.408 & $0.20803 \times 10-3$ & 0.153 & 0.784 & 86.28 & 2.30 & 2.94 & $\mathrm{C}$ & 16.25 \\
\hline 173.9 & 4940. & 0.498 & $0.21085 \times 10-3$ & 0.153 & 0.786 & 86.28 & 1.91 & 2.97 & $\mathrm{C}$ & 17.91 \\
\hline 180.7 & 4931. & 0.584 & $0.21389 \times 10-3$ & 0.153 & 0.787 & 86.28 & 1.64 & 2.99 & $\mathrm{C}$ & 20.94 \\
\hline 189.3 & 4922. & 0.675 & $0.21700 \times 10-3$ & 0.152 & 0.788 & 86.28 & 1.43 & 3.01 & $\mathrm{C}$ & 25.81 \\
\hline 201.2 & 4911. & 0.777 & $0.22040 \times 10-3$ & 0.152 & 0.790 & 86.28 & 1.24 & 3.04 & $\mathrm{C}$ & 32.83 \\
\hline 86.1 & 4790. & 0.067 & $0.84770 \times 10-4$ & 0.154 & 0.780 & 86.28 & 10.81 & 2.85 & $\mathrm{C}$ & 56.81 \\
\hline 94.4 & 4786. & 0.108 & $0.10133 \times 10-3$ & 0.154 & 0.780 & 86.28 & 7.49 & 2.86 & $\mathrm{C}$ & 22.90 \\
\hline 102.6 & 4781. & 0.153 & $0.11686 \times 10-3$ & 0.154 & 0.781 & 86.28 & 5.61 & 2.88 & $\mathrm{C}$ & 28.03 \\
\hline 110.8 & 4776. & 0.199 & $0.13132 \times 10-3$ & 0.154 & 0.781 & 86.28 & 4.45 & 2.89 & $\mathrm{C}$ & 22.32 \\
\hline 121.4 & 4770. & 0.261 & $0.14820 \times 10-3$ & 0.154 & 0.782 & 86.28 & 3.49 & 2.90 & $\mathrm{C}$ & 16.29 \\
\hline 128.0 & 4767. & 0.298 & $0.15788 \times 10-3$ & 0.154 & 0.783 & 86.28 & 3.09 & 2.91 & $\mathrm{C}$ & 20.78 \\
\hline 164.3 & 4751. & 0.391 & $0.20597 \times 10-3$ & 0.153 & 0.784 & 86.28 & 2.40 & 2.94 & $\mathrm{C}$ & 15.60 \\
\hline 167.0 & 4746. & 0.442 & $0.20744 \times 10-3$ & 0.153 & 0.785 & 86.28 & 2.14 & 2.95 & $\mathrm{C}$ & 16.43 \\
\hline 172.7 & 4738. & 0.532 & $0.20912 \times 10-3$ & 0.153 & 0.786 & 86.28 & 1.79 & 2.98 & $\mathrm{C}$ & 18.16 \\
\hline 179.4 & 4729. & 0.617 & $0.21106 \times 10-3$ & 0.152 & 0.787 & 86.28 & 1.55 & 3.00 & $\mathrm{C}$ & 21.17 \\
\hline 187.9 & 4720. & 0.707 & $0.21301 \times 10-3$ & 0.152 & 0.789 & 86.28 & 1.36 & 3.02 & $\mathrm{C}$ & 25.91 \\
\hline 199.3 & 4711. & 0.807 & $0.21510 \times 10-3$ & 0.152 & 0.790 & 86.28 & 1.20 & 3.05 & $\mathrm{C}$ & 32.86 \\
\hline 71.9 & 2941. & 0.085 & $0.12584 \times 10-3$ & 0.154 & 0.780 & 86.28 & 9.06 & 2.86 & $\mathrm{C}$ & 66.37 \\
\hline 77.8 & 2937. & 0.143 & $0.13598 \times 10-3$ & 0.154 & 0.781 & 86.28 & 5.95 & 2.87 & $\mathrm{C}$ & 20.35 \\
\hline 84.0 & 2934. & 0.200 & $0.14564 \times 10-3$ & 0.154 & 0.781 & 86.28 & 4.43 & 2.89 & $\mathrm{C}$ & 31.59 \\
\hline 90.5 & 2930. & 0.257 & $0.15467 \times 10-3$ & 0.154 & 0.782 & 86.28 & 3.54 & 2.90 & $\mathrm{C}$ & 27.24 \\
\hline 98.9 & 2926. & 0.327 & $0.16519 \times 10-3$ & 0.154 & 0.783 & 86.28 & 2.83 & 2.92 & $\mathrm{C}$ & 17.86 \\
\hline 104.3 & 2924. & 0.369 & $0.17138 \times 10-3$ & 0.154 & 0.784 & 86.28 & 2.54 & 2.93 & $\mathrm{C}$ & 34.69 \\
\hline 97.9 & 2918. & 0.452 & $0.15704 \times 10-3$ & 0.153 & 0.785 & 86.28 & 2.09 & 2.95 & $\mathrm{C}$ & 21.59 \\
\hline 100.0 & 2916. & 0.492 & $0.15801 \times 10-3$ & 0.153 & 0.786 & 86.28 & 1.93 & 2.96 & $\mathrm{C}$ & 22.23 \\
\hline 103.9 & 2913. & 0.560 & $0.15901 \times 10-3$ & 0.153 & 0.787 & 86.28 & 1.71 & 2.98 & $\mathrm{C}$ & 21.17 \\
\hline 107.7 & 2909. & 0.625 & $0.16015 \times 10-3$ & 0.153 & 0.788 & 86.28 & 1.54 & 3.00 & $\mathrm{C}$ & 24.08 \\
\hline 112.0 & 2906. & 0.692 & $0.16130 \times 10-3$ & 0.153 & 0.789 & 86.28 & 1.39 & 3.02 & $\mathrm{C}$ & 28.37 \\
\hline 117.0 & 2903. & 0.768 & $0.16253 \times 10-3$ & 0.153 & 0.790 & 86.28 & 1.26 & 3.03 & $\mathrm{C}$ & 34.90 \\
\hline 257.6 & 6546. & 0.173 & $0.47187 \times 10-3$ & 0.154 & 0.781 & 86.28 & 5.05 & 2.88 & $\mathrm{P}$ & 14.97 \\
\hline 266.2 & 6519. & 0.366 & $0.38913 \times 10-3$ & 0.153 & 0.784 & 86.28 & 2.55 & 2.93 & $\mathrm{P}$ & 20.11 \\
\hline 267.8 & 6501. & 0.513 & $0.31106 \times 10-3$ & 0.153 & 0.786 & 86.28 & 1.86 & 2.97 & $\mathrm{P}$ & 24.49 \\
\hline 255.8 & 6487. & 0.619 & $0.23637 \times 10-3$ & 0.153 & 0.788 & 86.28 & 1.55 & 3.00 & $\mathrm{P}$ & 34.44 \\
\hline 209.7 & 6474. & 0.707 & $0.14714 \times 10-3$ & 0.153 & 0.789 & 86.28 & 1.36 & 3.02 & $\mathrm{P}$ & 50.02 \\
\hline 160.3 & 6468. & 0.737 & $0.97110 \times 10-4$ & 0.152 & 0.789 & 86.28 & 1.31 & 3.03 & $\mathrm{P}$ & 51.03 \\
\hline 299.0 & 6428. & 0.797 & $0.14540 \times 10-3$ & 0.150 & 0.789 & 86.28 & 1.21 & 3.05 & $\mathrm{P}$ & 79.51 \\
\hline 314.7 & 6421. & 0.832 & $0.12557 \times 10-3$ & 0.150 & 0.790 & 86.28 & 1.17 & 3.06 & $\mathrm{P}$ & 53.74 \\
\hline 334.0 & 6410. & 0.880 & $0.91160 \times 10-4$ & 0.150 & 0.790 & 86.28 & 1.10 & 3.07 & $\mathrm{P}$ & 134.34 \\
\hline 319.1 & 6400. & 0.911 & $0.58840 \times 10-4$ & 0.149 & 0.790 & 86.28 & 1.07 & 3.08 & $\mathrm{P}$ & 134.72 \\
\hline 207.3 & 6390. & 0.929 & $0.25110 \times 10-4$ & 0.149 & 0.790 & 86.28 & 1.05 & 3.08 & $\mathrm{P}$ & 760.99 \\
\hline 267.5 & 9118. & 0.153 & $0.30654 \times 10-3$ & 0.153 & 0.780 & 86.28 & 5.59 & 2.88 & $\mathrm{P}$ & 17.22 \\
\hline 269.3 & 9090. & 0.279 & $0.25519 \times 10-3$ & 0.153 & 0.782 & 86.28 & 3.29 & 2.91 & $\mathrm{P}$ & 22.07 \\
\hline 263.9 & 9068. & 0.375 & $0.20709 \times 10-3$ & 0.152 & 0.783 & 86.28 & 2.50 & 2.94 & $\mathrm{P}$ & 25.63 \\
\hline
\end{tabular}




\begin{tabular}{|c|c|c|c|c|c|c|c|c|c|c|}
\hline 247.5 & 9049. & 0.446 & $0.16168 \times 10-3$ & 0.152 & 0.784 & 86.28 & 2.12 & 2.96 & $\mathrm{P}$ & 33.98 \\
\hline 206.4 & 9030. & 0.507 & $0.10799 \times 10-3$ & 0.152 & 0.785 & 86.28 & 1.88 & 2.98 & $\mathrm{P}$ & 47.38 \\
\hline 168.2 & 9020. & 0.530 & $0.78020 \times 10-4$ & 0.151 & 0.785 & 86.28 & 1.80 & 2.98 & $\mathrm{P}$ & 48.19 \\
\hline 300.3 & 8942. & 0.582 & $0.12281 \times 10-3$ & 0.148 & 0.784 & 86.28 & 1.65 & 3.00 & $\mathrm{P}$ & 65.27 \\
\hline 305.6 & 8929. & 0.611 & $0.10764 \times 10-3$ & 0.148 & 0.784 & 86.28 & 1.57 & 3.01 & $\mathrm{P}$ & 42.65 \\
\hline 304.9 & 8906. & 0.653 & $0.81470 \times 10-4$ & 0.147 & 0.785 & 86.28 & 1.47 & 3.02 & $\mathrm{P}$ & 96.76 \\
\hline 280.2 & 8886. & 0.681 & $0.56920 \times 10-4$ & 0.146 & 0.785 & 86.28 & 1.42 & 3.03 & $\mathrm{P}$ & 91.92 \\
\hline 206.2 & 8864. & 0.701 & $0.31290 \times 10-4$ & 0.146 & 0.785 & 86.28 & 1.38 & 3.04 & $\mathrm{P}$ & 756.67 \\
\hline 26.3 & 8841. & 0.709 & $0.299 \times 10-5$ & 0.145 & 0.784 & 86.28 & 1.36 & 3.05 & $\mathrm{P}$ & 761.50 \\
\hline 218.7 & 5128. & 0.154 & $0.45728 \times 10-3$ & 0.154 & 0.781 & 86.28 & 5.58 & 2.88 & $\mathrm{P}$ & 17.39 \\
\hline 225.6 & 5109. & 0.339 & $0.37313 \times 10-3$ & 0.154 & 0.783 & 86.28 & 2.74 & 2.92 & $\mathrm{P}$ & 24.72 \\
\hline 225.1 & 5096. & 0.479 & $0.29373 \times 10-3$ & 0.153 & 0.786 & 86.28 & 1.98 & 2.96 & $\mathrm{P}$ & 32.49 \\
\hline 211.4 & 5087. & 0.579 & $0.21791 \times 10-3$ & 0.153 & 0.787 & 86.28 & 1.65 & 2.99 & $\mathrm{P}$ & 45.54 \\
\hline 163.4 & 5079. & 0.657 & $0.12757 \times 10-3$ & 0.153 & 0.788 & 86.28 & 1.46 & 3.01 & $\mathrm{P}$ & 68.54 \\
\hline 113.6 & 5076. & 0.682 & $0.76940 \times 10-4$ & 0.153 & 0.789 & 86.28 & 1.41 & 3.01 & $\mathrm{P}$ & 69.41 \\
\hline 241.4 & 5055. & 0.731 & $0.12931 \times 10-3$ & 0.152 & 0.789 & 86.28 & 1.32 & 3.03 & $\mathrm{P}$ & 129.83 \\
\hline 249.8 & 5051. & 0.762 & $0.10977 \times 10-3$ & 0.151 & 0.789 & 86.28 & 1.27 & 3.04 & $\mathrm{P}$ & 69.26 \\
\hline 248.4 & 5045. & 0.804 & $0.75890 \times 10-4$ & 0.151 & 0.790 & 86.28 & 1.21 & 3.05 & $\mathrm{P}$ & 217.01 \\
\hline 202.4 & 5040. & 0.828 & $0.44080 \times 10-4$ & 0.151 & 0.790 & 86.28 & 1.17 & 3.06 & $\mathrm{P}$ & 192.48 \\
\hline 66.8 & 5035. & 0.839 & $0.10920 \times 10-4$ & 0.151 & 0.790 & 86.28 & 1.16 & 3.06 & $\mathrm{P}$ & 421.50 \\
\hline 160.6 & 2759. & 0.201 & $0.53438 \times 10-3$ & 0.153 & 0.781 & 86.28 & 4.41 & 2.89 & $\mathrm{P}$ & 24.97 \\
\hline 173.6 & 2748. & 0.418 & $0.42870 \times 10-3$ & 0.153 & 0.784 & 86.28 & 2.25 & 2.95 & $\mathrm{P}$ & 40.66 \\
\hline 183.3 & 2742. & 0.578 & $0.32848 \times 10-3$ & 0.153 & 0.787 & 86.28 & 1.66 & 2.99 & $\mathrm{P}$ & 64.38 \\
\hline 181.3 & 2738. & 0.687 & $0.23230 \times 10-3$ & 0.153 & 0.789 & 86.28 & 1.40 & 3.02 & $\mathrm{P}$ & 82.69 \\
\hline 139.5 & 2736. & 0.767 & $0.11744 \times 10-3$ & 0.153 & 0.790 & 86.28 & 1.26 & 3.03 & $\mathrm{P}$ & 128.68 \\
\hline 78.7 & 2736. & 0.788 & $0.52990 \times 10-4$ & 0.153 & 0.791 & 86.28 & 1.23 & 3.04 & $\mathrm{P}$ & 130.88 \\
\hline 326.5 & 2731. & 0.831 & $0.13282 \times 10-3$ & 0.153 & 0.791 & 86.28 & 1.17 & 3.05 & $\mathrm{P}$ & 657.55 \\
\hline 436.8 & 2730. & 0.862 & $0.10752 \times 10-3$ & 0.153 & 0.792 & 86.28 & 1.13 & 3.06 & $\mathrm{P}$ & 144.63 \\
\hline 1589.5 & 2729. & 0.900 & $0.63690 \times 10-4$ & 0.152 & 0.792 & 86.28 & 1.08 & 3.07 & $\mathrm{P}$ & 1434.42 \\
\hline
\end{tabular}




\section{APPENDIX A2}

Convective Boiling of R449A within a micro-fin tube

(file: TaevapGWP3.dat)

\begin{tabular}{|c|c|c|c|c|c|c|c|c|c|c|}
\hline $\mathrm{Nu}$ & $\operatorname{Re}$ & $x_{\mathrm{q}}$ & Bo & $P_{\mathrm{S}} / P_{\mathrm{c}}$ & $T_{\mathrm{S}} / T_{\mathrm{c}}$ & $M_{\mathrm{w}}$ & $S v$ & $\operatorname{Pr}$ & flow & $U_{\mathrm{Nu}}$ \\
\hline 49.1 & 7063. & 0.083 & $0.24000 \times 10-4$ & 0.160 & 0.784 & 87.21 & 9.41 & 2.86 & $\mathrm{C}$ & 213.72 \\
\hline 79.9 & 7069. & 0.099 & $0.48700 \times 10-4$ & 0.160 & 0.784 & 87.21 & 8.18 & 2.86 & $\mathrm{C}$ & 35.72 \\
\hline 101.7 & 7079 . & 0.123 & $0.71570 \times 10-4$ & 0.160 & 0.785 & 87.21 & 6.82 & 2.86 & $\mathrm{C}$ & 39.94 \\
\hline 119.3 & 7093. & 0.154 & $0.92650 \times 10-4$ & 0.160 & 0.785 & 87.21 & 5.64 & 2.86 & $\mathrm{C}$ & 28.92 \\
\hline 139.1 & 7113. & 0.199 & $0.11708 \times 10-3$ & 0.160 & 0.786 & 87.21 & 4.50 & 2.86 & $\mathrm{C}$ & 18.36 \\
\hline 150.0 & 7126. & 0.228 & $0.13067 \times 10-3$ & 0.160 & 0.786 & 87.21 & 3.97 & 2.85 & $\mathrm{C}$ & 25.98 \\
\hline 213.4 & 7152. & 0.310 & $0.19716 \times 10-3$ & 0.159 & 0.787 & 87.21 & 2.99 & 2.85 & $\mathrm{C}$ & 21.95 \\
\hline 221.1 & 7173. & 0.359 & $0.20555 \times 10-3$ & 0.159 & 0.788 & 87.21 & 2.61 & 2.85 & $\mathrm{C}$ & 19.07 \\
\hline 236.3 & 7210. & 0.448 & $0.21983 \times 10-3$ & 0.158 & 0.789 & 87.21 & 2.12 & 2.84 & $\mathrm{C}$ & 20.80 \\
\hline 253.2 & 7247. & 0.536 & $0.23332 \times 10-3$ & 0.158 & 0.790 & 87.21 & 1.78 & 2.84 & $\mathrm{C}$ & 23.69 \\
\hline 274.1 & 7286. & 0.634 & $0.24734 \times 10-3$ & 0.158 & 0.791 & 87.21 & 1.52 & 2.84 & $\mathrm{C}$ & 29.29 \\
\hline 302.3 & 7331. & 0.748 & $0.26285 \times 10-3$ & 0.157 & 0.793 & 87.21 & 1.29 & 2.83 & $\mathrm{C}$ & 38.05 \\
\hline 155.6 & 9125. & 0.085 & $0.39370 \times 10-4$ & 0.160 & 0.784 & 87.21 & 9.26 & 2.86 & $\mathrm{C}$ & 86.90 \\
\hline 152.0 & 9135. & 0.105 & $0.52290 \times 10-4$ & 0.160 & 0.785 & 87.21 & 7.81 & 2.86 & $\mathrm{C}$ & 47.30 \\
\hline 152.8 & 9147. & 0.129 & $0.64240 \times 10-4$ & 0.159 & 0.785 & 87.21 & 6.59 & 2.86 & $\mathrm{C}$ & 36.28 \\
\hline 155.3 & 9161. & 0.155 & $0.75260 \times 10-4$ & 0.159 & 0.785 & 87.21 & 5.63 & 2.86 & $\mathrm{C}$ & 28.88 \\
\hline 159.7 & 9179. & 0.190 & $0.88020 \times 10-4$ & 0.159 & 0.786 & 87.21 & 4.69 & 2.86 & $\mathrm{C}$ & 21.27 \\
\hline 162.6 & 9191. & 0.212 & $0.95110 \times 10-4$ & 0.159 & 0.786 & 87.21 & 4.25 & 2.85 & $\mathrm{C}$ & 22.38 \\
\hline 229.9 & 9207. & 0.273 & $0.15151 \times 10-3$ & 0.158 & 0.786 & 87.21 & 3.37 & 2.85 & $\mathrm{C}$ & 23.30 \\
\hline 233.7 & 9226. & 0.311 & $0.15875 \times 10-3$ & 0.158 & 0.787 & 87.21 & 2.99 & 2.85 & $\mathrm{C}$ & 19.55 \\
\hline 240.7 & 9260. & 0.380 & $0.17114 \times 10-3$ & 0.158 & 0.788 & 87.21 & 2.48 & 2.85 & $\mathrm{C}$ & 20.90 \\
\hline 248.0 & 9291. & 0.449 & $0.18278 \times 10-3$ & 0.157 & 0.788 & 87.21 & 2.11 & 2.85 & $\mathrm{C}$ & 22.84 \\
\hline 256.0 & 9323. & 0.526 & $0.19485 \times 10-3$ & 0.157 & 0.789 & 87.21 & 1.82 & 2.84 & $\mathrm{C}$ & 26.72 \\
\hline 265.6 & 9359. & 0.616 & $0.20816 \times 10-3$ & 0.156 & 0.790 & 87.21 & 1.56 & 2.84 & $\mathrm{C}$ & 32.88 \\
\hline 88.6 & 4970. & 0.017 & $0.76950 \times 10-4$ & 0.162 & 0.785 & 87.21 & 25.05 & 2.86 & $\mathrm{C}$ & 64.09 \\
\hline 95.4 & 4982. & 0.054 & $0.93160 \times 10-4$ & 0.162 & 0.785 & 87.21 & 12.98 & 2.86 & $\mathrm{C}$ & 30.57 \\
\hline 101.8 & 4995. & 0.094 & $0.10821 \times 10-3$ & 0.162 & 0.786 & 87.21 & 8.49 & 2.86 & $\mathrm{C}$ & 30.76 \\
\hline 108.2 & 5008. & 0.137 & $0.12210 \times 10-3$ & 0.162 & 0.786 & 87.21 & 6.23 & 2.85 & $\mathrm{C}$ & 27.20 \\
\hline 116.4 & 5027. & 0.193 & $0.13820 \times 10-3$ & 0.162 & 0.787 & 87.21 & 4.62 & 2.85 & $\mathrm{C}$ & 17.89 \\
\hline 121.3 & 5038. & 0.227 & $0.14719 \times 10-3$ & 0.162 & 0.788 & 87.21 & 3.99 & 2.85 & $\mathrm{C}$ & 25.94 \\
\hline 165.1 & 5061. & 0.315 & $0.20814 \times 10-3$ & 0.161 & 0.789 & 87.21 & 2.95 & 2.84 & $\mathrm{C}$ & 21.63 \\
\hline 167.1 & 5077. & 0.366 & $0.21086 \times 10-3$ & 0.161 & 0.790 & 87.21 & 2.56 & 2.84 & $\mathrm{C}$ & 19.18 \\
\hline 171.3 & 5105. & 0.454 & $0.21533 \times 10-3$ & 0.161 & 0.791 & 87.21 & 2.09 & 2.84 & $\mathrm{C}$ & 20.85 \\
\hline 175.7 & 5132. & 0.539 & $0.21963 \times 10-3$ & 0.161 & 0.792 & 87.21 & 1.77 & 2.83 & $\mathrm{C}$ & 23.85 \\
\hline 181.3 & 5159. & 0.629 & $0.22408 \times 10-3$ & 0.161 & 0.793 & 87.21 & 1.53 & 2.83 & $\mathrm{C}$ & 29.02 \\
\hline 188.8 & 5189. & 0.730 & $0.22898 \times 10-3$ & 0.160 & 0.794 & 87.21 & 1.32 & 2.82 & $\mathrm{C}$ & 37.50 \\
\hline 36.2 & 3169. & 0.009 & $0.47840 \times 10-4$ & 0.161 & 0.784 & 87.21 & 30.93 & 2.86 & $\mathrm{C}$ & 986.77 \\
\hline 51.0 & 3174. & 0.036 & $0.73500 \times 10-4$ & 0.161 & 0.784 & 87.21 & 17.01 & 2.86 & $\mathrm{C}$ & 28.09 \\
\hline 63.9 & 3181. & 0.070 & $0.97270 \times 10-4$ & 0.161 & 0.785 & 87.21 & 10.72 & 2.86 & $\mathrm{C}$ & 39.63 \\
\hline
\end{tabular}




\begin{tabular}{|c|c|c|c|c|c|c|c|c|c|c|}
\hline 75.7 & 3189. & 0.110 & $0.11921 \times 10-3$ & 0.161 & 0.785 & 87.21 & 7.49 & 2.86 & $\mathrm{C}$ & 30.25 \\
\hline 89.9 & 3201. & 0.167 & $0.14463 \times 10-3$ & 0.161 & 0.786 & 87.21 & 5.26 & 2.85 & $\mathrm{C}$ & 19.26 \\
\hline 98.5 & 3208. & 0.203 & $0.15882 \times 10-3$ & 0.161 & 0.787 & 87.21 & 4.42 & 2.85 & $\mathrm{C}$ & 31.20 \\
\hline 124.7 & 3226. & 0.292 & $0.19893 \times 10-3$ & 0.161 & 0.788 & 87.21 & 3.17 & 2.85 & $\mathrm{C}$ & 24.45 \\
\hline 127.8 & 3236. & 0.340 & $0.20004 \times 10-3$ & 0.161 & 0.789 & 87.21 & 2.75 & 2.84 & $\mathrm{C}$ & 21.24 \\
\hline 133.6 & 3254. & 0.423 & $0.20170 \times 10-3$ & 0.161 & 0.790 & 87.21 & 2.23 & 2.84 & $\mathrm{C}$ & 23.19 \\
\hline 139.9 & 3271. & 0.502 & $0.20336 \times 10-3$ & 0.161 & 0.791 & 87.21 & 1.90 & 2.83 & $\mathrm{C}$ & 27.69 \\
\hline 147.4 & 3288. & 0.584 & $0.20506 \times 10-3$ & 0.160 & 0.792 & 87.21 & 1.64 & 2.83 & $\mathrm{C}$ & 33.35 \\
\hline 157.1 & 3307. & 0.676 & $0.20692 \times 10-3$ & 0.160 & 0.794 & 87.21 & 1.43 & 2.82 & $\mathrm{C}$ & 44.66 \\
\hline 279.4 & 6866. & 0.142 & $0.55538 \times 10-3$ & 0.161 & 0.786 & 87.21 & 6.06 & 2.86 & $\mathrm{P}$ & 11.38 \\
\hline 288.5 & 6962. & 0.361 & $0.44731 \times 10-3$ & 0.161 & 0.789 & 87.21 & 2.59 & 2.84 & $\mathrm{P}$ & 14.93 \\
\hline 288.2 & 7033. & 0.523 & $0.34649 \times 10-3$ & 0.161 & 0.791 & 87.21 & 1.83 & 2.83 & $\mathrm{P}$ & 17.98 \\
\hline 271.1 & 7081. & 0.635 & $0.25239 \times 10-3$ & 0.160 & 0.793 & 87.21 & 1.51 & 2.83 & $\mathrm{P}$ & 24.67 \\
\hline 210.5 & 7116. & 0.721 & $0.14234 \times 10-3$ & 0.160 & 0.794 & 87.21 & 1.34 & 2.82 & $\mathrm{P}$ & 35.58 \\
\hline 143.7 & 7125. & 0.748 & $0.81100 \times 10-4$ & 0.160 & 0.794 & 87.21 & 1.29 & 2.82 & $\mathrm{P}$ & 37.68 \\
\hline 311.3 & 7109. & 0.797 & $0.13115 \times 10-3$ & 0.157 & 0.794 & 87.21 & 1.22 & 2.83 & $\mathrm{P}$ & 57.27 \\
\hline 353.8 & 7118. & 0.827 & $0.11324 \times 10-3$ & 0.157 & 0.794 & 87.21 & 1.17 & 2.82 & $\mathrm{P}$ & 52.08 \\
\hline 490.5 & 7129. & 0.868 & $0.82610 \times 10-4$ & 0.157 & 0.795 & 87.21 & 1.12 & 2.82 & $\mathrm{P}$ & 117.44 \\
\hline 1018.4 & 7133. & 0.895 & $0.53970 \times 10-4$ & 0.156 & 0.795 & 87.21 & 1.09 & 2.82 & $\mathrm{P}$ & 289.47 \\
\hline 279.9 & 6910. & 0.141 & $0.55385 \times 10-3$ & 0.161 & 0.786 & 87.21 & 6.09 & 2.86 & $\mathrm{P}$ & 11.36 \\
\hline 289.1 & 7006. & 0.360 & $0.44616 \times 10-3$ & 0.161 & 0.789 & 87.21 & 2.60 & 2.84 & $\mathrm{P}$ & 14.92 \\
\hline 289.0 & 7077. & 0.521 & $0.34570 \times 10-3$ & 0.161 & 0.791 & 87.21 & 1.83 & 2.83 & $\mathrm{P}$ & 17.99 \\
\hline 272.3 & 7126. & 0.633 & $0.25195 \times 10-3$ & 0.161 & 0.793 & 87.21 & 1.52 & 2.83 & $\mathrm{P}$ & 24.72 \\
\hline 212.3 & 7161. & 0.719 & $0.14230 \times 10-3$ & 0.160 & 0.794 & 87.21 & 1.34 & 2.82 & $\mathrm{P}$ & 35.66 \\
\hline 145.7 & 7170. & 0.746 & $0.81290 \times 10-4$ & 0.160 & 0.794 & 87.21 & 1.29 & 2.82 & $\mathrm{P}$ & 37.79 \\
\hline 314.5 & 7153. & 0.794 & $0.13083 \times 10-3$ & 0.158 & 0.794 & 87.21 & 1.22 & 2.82 & $\mathrm{P}$ & 57.16 \\
\hline 359.1 & 7163. & 0.825 & $0.11318 \times 10-3$ & 0.157 & 0.794 & 87.21 & 1.18 & 2.82 & $\mathrm{P}$ & 52.28 \\
\hline 505.6 & 7174. & 0.866 & $0.82990 \times 10-4$ & 0.157 & 0.795 & 87.21 & 1.12 & 2.82 & $\mathrm{P}$ & 118.53 \\
\hline 1114.6 & 7178. & 0.893 & $0.54760 \times 10-4$ & 0.156 & 0.795 & 87.21 & 1.09 & 2.82 & $\mathrm{P}$ & 308.03 \\
\hline 279.1 & 9116. & 0.100 & $0.39279 \times 10-3$ & 0.161 & 0.785 & 87.21 & 8.10 & 2.86 & $\mathrm{P}$ & 12.66 \\
\hline 280.6 & 9202. & 0.256 & $0.31843 \times 10-3$ & 0.161 & 0.788 & 87.21 & 3.58 & 2.85 & $\mathrm{P}$ & 16.06 \\
\hline 273.0 & 9265. & 0.371 & $0.24921 \times 10-3$ & 0.161 & 0.789 & 87.21 & 2.53 & 2.84 & $\mathrm{P}$ & 18.72 \\
\hline 251.3 & 9307. & 0.452 & $0.18484 \times 10-3$ & 0.160 & 0.790 & 87.21 & 2.10 & 2.84 & $\mathrm{P}$ & 24.21 \\
\hline 195.0 & 9336. & 0.516 & $0.10978 \times 10-3$ & 0.160 & 0.791 & 87.21 & 1.85 & 2.84 & $\mathrm{P}$ & 33.11 \\
\hline 140.8 & 9343. & 0.538 & $0.68080 \times 10-4$ & 0.160 & 0.791 & 87.21 & 1.78 & 2.83 & $\mathrm{P}$ & 34.67 \\
\hline 277.8 & 9299. & 0.581 & $0.11037 \times 10-3$ & 0.157 & 0.790 & 87.21 & 1.65 & 2.84 & $\mathrm{P}$ & 48.95 \\
\hline 297.8 & 9306. & 0.607 & $0.96280 \times 10-4$ & 0.156 & 0.790 & 87.21 & 1.58 & 2.84 & $\mathrm{P}$ & 41.37 \\
\hline 342.0 & 9311. & 0.643 & $0.72290 \times 10-4$ & 0.155 & 0.790 & 87.21 & 1.50 & 2.84 & $\mathrm{P}$ & 85.55 \\
\hline 406.4 & 9309. & 0.667 & $0.49860 \times 10-4$ & 0.155 & 0.790 & 87.21 & 1.45 & 2.84 & $\mathrm{P}$ & 113.06 \\
\hline 566.0 & 9300. & 0.683 & $0.26590 \times 10-4$ & 0.154 & 0.790 & 87.21 & 1.41 & 2.84 & $\mathrm{P}$ & 701.40 \\
\hline 280.7 & 9190. & 0.100 & $0.39346 \times 10-3$ & 0.162 & 0.786 & 87.21 & 8.13 & 2.86 & $\mathrm{P}$ & 12.63 \\
\hline 282.8 & 9277. & 0.255 & $0.31885 \times 10-3$ & 0.161 & 0.788 & 87.21 & 3.58 & 2.85 & $\mathrm{P}$ & 16.07 \\
\hline 275.2 & 9341. & 0.371 & $0.24940 \times 10-3$ & 0.161 & 0.789 & 87.21 & 2.53 & 2.84 & $\mathrm{P}$ & 18.71 \\
\hline 253.8 & 9383. & 0.452 & $0.18482 \times 10-3$ & 0.161 & 0.790 & 87.21 & 2.10 & 2.84 & $\mathrm{P}$ & 24.27 \\
\hline 197.3 & 9412. & 0.516 & $0.10952 \times 10-3$ & 0.160 & 0.791 & 87.21 & 1.85 & 2.83 & $\mathrm{P}$ & 33.36 \\
\hline 142.5 & 9419. & 0.538 & $0.67680 \times 10-4$ & 0.160 & 0.791 & 87.21 & 1.78 & 2.83 & $\mathrm{P}$ & 34.98 \\
\hline 283.9 & 9375. & 0.581 & $0.11027 \times 10-3$ & 0.157 & 0.790 & 87.21 & 1.65 & 2.84 & $\mathrm{P}$ & 48.85 \\
\hline 305.5 & 9382. & 0.606 & $0.96180 \times 10-4$ & 0.157 & 0.790 & 87.21 & 1.58 & 2.84 & $\mathrm{P}$ & 42.07 \\
\hline 355.9 & 9387. & 0.642 & $0.72200 \times 10-4$ & 0.156 & 0.791 & 87.21 & 1.50 & 2.84 & $\mathrm{P}$ & 86.38 \\
\hline
\end{tabular}




\begin{tabular}{|c|c|c|c|c|c|c|c|c|c|c|}
\hline 435.1 & 9386. & 0.667 & $0.49770 \times 10-4$ & 0.155 & 0.790 & 87.21 & 1.45 & 2.84 & $\mathrm{P}$ & 118.30 \\
\hline 676.2 & 9376. & 0.683 & $0.26490 \times 10-4$ & 0.154 & 0.790 & 87.21 & 1.41 & 2.84 & $\mathrm{P}$ & 740.67 \\
\hline-35.5 & 9358. & 0.690 & $0.86 \times 10-6$ & 0.153 & 0.790 & 87.21 & 1.40 & 2.84 & $\mathrm{P}$ & 904.52 \\
\hline 249.3 & 4835. & 0.159 & $0.62334 \times 10-3$ & 0.162 & 0.786 & 87.21 & 5.49 & 2.85 & $\mathrm{P}$ & 11.95 \\
\hline 266.8 & 4913. & 0.404 & $0.49971 \times 10-3$ & 0.161 & 0.790 & 87.21 & 2.33 & 2.84 & $\mathrm{P}$ & 17.15 \\
\hline 278.3 & 4971. & 0.584 & $0.38427 \times 10-3$ & 0.161 & 0.793 & 87.21 & 1.64 & 2.83 & $\mathrm{P}$ & 21.18 \\
\hline 274.8 & 5011. & 0.709 & $0.27636 \times 10-3$ & 0.161 & 0.795 & 87.21 & 1.36 & 2.82 & $\mathrm{P}$ & 30.89 \\
\hline 224.8 & 5040. & 0.802 & $0.15000 \times 10-3$ & 0.161 & 0.796 & 87.21 & 1.21 & 2.82 & $\mathrm{P}$ & 48.41 \\
\hline 151.5 & 5048. & 0.830 & $0.79650 \times 10-4$ & 0.161 & 0.796 & 87.21 & 1.17 & 2.82 & $\mathrm{P}$ & 52.42 \\
\hline 398.4 & 5047. & 0.876 & $0.13142 \times 10-3$ & 0.159 & 0.796 & 87.21 & 1.11 & 2.81 & $\mathrm{P}$ & 94.21 \\
\hline 547.6 & 5055. & 0.906 & $0.11193 \times 10-3$ & 0.159 & 0.797 & 87.21 & 1.07 & 2.81 & $\mathrm{P}$ & 97.88 \\
\hline 3308.8 & 5066. & 0.946 & $0.78470 \times 10-4$ & 0.159 & 0.797 & 87.21 & 1.03 & 2.81 & $\mathrm{P}$ & 770.22 \\
\hline 174.3 & 2966. & 0.159 & $0.62103 \times 10-3$ & 0.163 & 0.787 & 87.21 & 5.47 & 2.85 & $\mathrm{P}$ & 15.11 \\
\hline 188.4 & 3014. & 0.402 & $0.49028 \times 10-3$ & 0.163 & 0.791 & 87.21 & 2.34 & 2.84 & $\mathrm{P}$ & 25.14 \\
\hline 197.3 & 3049. & 0.577 & $0.36818 \times 10-3$ & 0.163 & 0.793 & 87.21 & 1.66 & 2.83 & $\mathrm{P}$ & 32.13 \\
\hline 193.1 & 3073. & 0.694 & $0.25410 \times 10-3$ & 0.163 & 0.795 & 87.21 & 1.39 & 2.82 & $\mathrm{P}$ & 49.69 \\
\hline 143.0 & 3090. & 0.775 & $0.12061 \times 10-3$ & 0.163 & 0.797 & 87.21 & 1.25 & 2.81 & $\mathrm{P}$ & 84.09 \\
\hline 70.2 & 3094. & 0.796 & $0.46330 \times 10-4$ & 0.163 & 0.797 & 87.21 & 1.22 & 2.81 & $\mathrm{P}$ & 87.86 \\
\hline 301.8 & 3098. & 0.828 & $0.11373 \times 10-3$ & 0.162 & 0.797 & 87.21 & 1.17 & 2.81 & $\mathrm{P}$ & 207.42 \\
\hline 433.6 & 3103. & 0.853 & $0.93320 \times 10-4$ & 0.162 & 0.797 & 87.21 & 1.14 & 2.81 & $\mathrm{P}$ & 149.37 \\
\hline 63.1 & 6755. & 0.004 & $0.29570 \times 10-4$ & 0.163 & 0.785 & 87.21 & 36.41 & 2.86 & $\mathrm{C}$ & 253.96 \\
\hline 84.6 & 6762. & 0.022 & $0.50000 \times 10-4$ & 0.163 & 0.785 & 87.21 & 22.29 & 2.86 & $\mathrm{C}$ & 37.61 \\
\hline 100.0 & 6772. & 0.046 & $0.68900 \times 10-4$ & 0.163 & 0.785 & 87.21 & 14.52 & 2.86 & $\mathrm{C}$ & 34.37 \\
\hline 112.6 & 6784. & 0.074 & $0.86330 \times 10-4$ & 0.163 & 0.786 & 87.21 & 10.23 & 2.85 & $\mathrm{C}$ & 26.65 \\
\hline 126.7 & 6802. & 0.116 & $0.10652 \times 10-3$ & 0.163 & 0.786 & 87.21 & 7.18 & 2.85 & $\mathrm{C}$ & 19.03 \\
\hline 134.7 & 6814. & 0.142 & $0.11775 \times 10-3$ & 0.163 & 0.787 & 87.21 & 6.02 & 2.85 & $\mathrm{C}$ & 21.83 \\
\hline 234.6 & 6841. & 0.225 & $0.21756 \times 10-3$ & 0.162 & 0.788 & 87.21 & 4.02 & 2.85 & $\mathrm{C}$ & 22.59 \\
\hline 245.5 & 6864. & 0.279 & $0.22834 \times 10-3$ & 0.162 & 0.789 & 87.21 & 3.30 & 2.85 & $\mathrm{C}$ & 19.36 \\
\hline 266.5 & 6905. & 0.378 & $0.24671 \times 10-3$ & 0.162 & 0.790 & 87.21 & 2.49 & 2.84 & $\mathrm{C}$ & 22.00 \\
\hline 289.6 & 6945. & 0.477 & $0.26407 \times 10-3$ & 0.161 & 0.791 & 87.21 & 1.99 & 2.83 & $\mathrm{C}$ & 25.09 \\
\hline 318.3 & 6989. & 0.588 & $0.28212 \times 10-3$ & 0.161 & 0.793 & 87.21 & 1.63 & 2.83 & $\mathrm{C}$ & 31.22 \\
\hline 356.7 & 7040. & 0.718 & $0.30211 \times 10-3$ & 0.160 & 0.794 & 87.21 & 1.34 & 2.82 & $\mathrm{C}$ & 40.95 \\
\hline 110.9 & 6795. & 0.010 & $0.49340 \times 10-4$ & 0.164 & 0.785 & 87.21 & 30.13 & 2.86 & $\mathrm{C}$ & 65.10 \\
\hline 114.6 & 6805. & 0.035 & $0.65260 \times 10-4$ & 0.164 & 0.786 & 87.21 & 17.17 & 2.86 & $\mathrm{C}$ & 41.12 \\
\hline 119.2 & 6817. & 0.064 & $0.80000 \times 10-4$ & 0.164 & 0.786 & 87.21 & 11.42 & 2.85 & $\mathrm{C}$ & 30.52 \\
\hline 124.3 & 6831. & 0.096 & $0.93590 \times 10-4$ & 0.164 & 0.787 & 87.21 & 8.34 & 2.85 & $\mathrm{C}$ & 24.90 \\
\hline 130.9 & 6850. & 0.140 & $0.10933 \times 10-3$ & 0.164 & 0.787 & 87.21 & 6.11 & 2.85 & $\mathrm{C}$ & 19.64 \\
\hline 135.0 & 6862. & 0.167 & $0.11810 \times 10-3$ & 0.164 & 0.788 & 87.21 & 5.24 & 2.85 & $\mathrm{C}$ & 19.10 \\
\hline 229.8 & 6889. & 0.249 & $0.21613 \times 10-3$ & 0.163 & 0.789 & 87.21 & 3.66 & 2.85 & $\mathrm{C}$ & 20.59 \\
\hline 239.9 & 6912. & 0.302 & $0.22774 \times 10-3$ & 0.163 & 0.789 & 87.21 & 3.06 & 2.84 & $\mathrm{C}$ & 19.37 \\
\hline 259.4 & 6954. & 0.401 & $0.24755 \times 10-3$ & 0.163 & 0.791 & 87.21 & 2.35 & 2.84 & $\mathrm{C}$ & 21.55 \\
\hline 280.7 & 6994. & 0.502 & $0.26625 \times 10-3$ & 0.162 & 0.792 & 87.21 & 1.90 & 2.83 & $\mathrm{C}$ & 25.00 \\
\hline 306.3 & 7039. & 0.613 & $0.28571 \times 10-3$ & 0.162 & 0.793 & 87.21 & 1.56 & 2.83 & $\mathrm{C}$ & 30.96 \\
\hline 340.8 & 7090. & 0.746 & $0.30726 \times 10-3$ & 0.161 & 0.795 & 87.21 & 1.29 & 2.82 & $\mathrm{C}$ & 39.90 \\
\hline 116.3 & 9338. & 0.005 & $0.27690 \times 10-4$ & 0.163 & 0.785 & 87.21 & 35.00 & 2.86 & $\mathrm{C}$ & 82.64 \\
\hline 120.5 & 9345. & 0.020 & $0.39530 \times 10-4$ & 0.163 & 0.785 & 87.21 & 23.09 & 2.86 & $\mathrm{C}$ & 48.25 \\
\hline 124.9 & 9355. & 0.038 & $0.50500 \times 10-4$ & 0.163 & 0.785 & 87.21 & 16.25 & 2.86 & $\mathrm{C}$ & 34.54 \\
\hline 129.2 & 9366. & 0.059 & $0.60600 \times 10-4$ & 0.163 & 0.786 & 87.21 & 12.16 & 2.86 & $\mathrm{C}$ & 27.56 \\
\hline 134.7 & 9382. & 0.087 & $0.72290 \times 10-4$ & 0.163 & 0.786 & 87.21 & 9.02 & 2.86 & $\mathrm{C}$ & 21.20 \\
\hline
\end{tabular}




\begin{tabular}{|c|c|c|c|c|c|c|c|c|c|c|}
\hline 137.9 & 9392. & 0.105 & $0.78780 \times 10-4$ & 0.163 & 0.786 & 87.21 & 7.76 & 2.85 & $\mathrm{C}$ & 20.50 \\
\hline 242.4 & 9410. & 0.163 & $0.15578 \times 10-3$ & 0.162 & 0.787 & 87.21 & 5.36 & 2.85 & $\mathrm{C}$ & 24.26 \\
\hline 252.5 & 9432. & 0.202 & $0.16681 \times 10-3$ & 0.162 & 0.787 & 87.21 & 4.43 & 2.85 & $\mathrm{C}$ & 19.84 \\
\hline 269.8 & 9470. & 0.275 & $0.18570 \times 10-3$ & 0.161 & 0.788 & 87.21 & 3.34 & 2.85 & $\mathrm{C}$ & 21.42 \\
\hline 287.1 & 9507. & 0.351 & $0.20344 \times 10-3$ & 0.161 & 0.789 & 87.21 & 2.66 & 2.84 & $\mathrm{C}$ & 23.19 \\
\hline 305.6 & 9548. & 0.437 & $0.22187 \times 10-3$ & 0.160 & 0.790 & 87.21 & 2.16 & 2.84 & $\mathrm{C}$ & 27.34 \\
\hline 328.0 & 9594. & 0.541 & $0.24220 \times 10-3$ & 0.160 & 0.791 & 87.21 & 1.77 & 2.83 & $\mathrm{C}$ & 33.58 \\
\hline 99.2 & 9148. & 0.005 & $0.26790 \times 10-4$ & 0.163 & 0.785 & 87.21 & 35.33 & 2.86 & $\mathrm{C}$ & 76.04 \\
\hline 110.5 & 9155. & 0.020 & $0.39240 \times 10-4$ & 0.163 & 0.785 & 87.21 & 23.37 & 2.86 & $\mathrm{C}$ & 45.93 \\
\hline 119.3 & 9164. & 0.038 & $0.50760 \times 10-4$ & 0.163 & 0.785 & 87.21 & 16.39 & 2.86 & $\mathrm{C}$ & 33.96 \\
\hline 126.6 & 9175. & 0.058 & $0.61370 \times 10-4$ & 0.163 & 0.786 & 87.21 & 12.21 & 2.86 & $\mathrm{C}$ & 27.26 \\
\hline 135.1 & 9191. & 0.087 & $0.73650 \times 10-4$ & 0.163 & 0.786 & 87.21 & 9.01 & 2.85 & $\mathrm{C}$ & 21.40 \\
\hline 139.8 & 9202. & 0.106 & $0.80470 \times 10-4$ & 0.163 & 0.786 & 87.21 & 7.73 & 2.85 & $\mathrm{C}$ & 20.69 \\
\hline 244.7 & 9220. & 0.164 & $0.15597 \times 10-3$ & 0.162 & 0.787 & 87.21 & 5.34 & 2.85 & $\mathrm{C}$ & 22.84 \\
\hline 259.5 & 9241. & 0.203 & $0.16883 \times 10-3$ & 0.162 & 0.787 & 87.21 & 4.41 & 2.85 & $\mathrm{C}$ & 20.30 \\
\hline 285.7 & 9280. & 0.278 & $0.19087 \times 10-3$ & 0.162 & 0.788 & 87.21 & 3.31 & 2.85 & $\mathrm{C}$ & 21.77 \\
\hline 311.5 & 9318. & 0.356 & $0.21156 \times 10-3$ & 0.161 & 0.789 & 87.21 & 2.63 & 2.84 & $\mathrm{C}$ & 24.33 \\
\hline 340.8 & 9360. & 0.446 & $0.23306 \times 10-3$ & 0.161 & 0.790 & 87.21 & 2.12 & 2.84 & $\mathrm{C}$ & 29.22 \\
\hline 376.8 & 9410. & 0.556 & $0.25680 \times 10-3$ & 0.160 & 0.792 & 87.21 & 1.72 & 2.83 & $\mathrm{C}$ & 36.37 \\
\hline 87.6 & 5135. & 0.012 & $0.55130 \times 10-4$ & 0.162 & 0.784 & 87.21 & 28.39 & 2.86 & $\mathrm{C}$ & 54.88 \\
\hline 93.8 & 5144. & 0.040 & $0.71010 \times 10-4$ & 0.162 & 0.785 & 87.21 & 15.94 & 2.86 & $\mathrm{C}$ & 35.61 \\
\hline 99.9 & 5154. & 0.071 & $0.85730 \times 10-4$ & 0.162 & 0.785 & 87.21 & 10.59 & 2.86 & $\mathrm{C}$ & 29.35 \\
\hline 106.0 & 5165. & 0.105 & $0.99310 \times 10-4$ & 0.162 & 0.786 & 87.21 & 7.76 & 2.86 & $\mathrm{C}$ & 25.26 \\
\hline 113.9 & 5180. & 0.151 & $0.11503 \times 10-3$ & 0.162 & 0.786 & 87.21 & 5.71 & 2.85 & $\mathrm{C}$ & 19.57 \\
\hline 118.9 & 5190. & 0.180 & $0.12380 \times 10-3$ & 0.161 & 0.787 & 87.21 & 4.92 & 2.85 & $\mathrm{C}$ & 20.43 \\
\hline 197.5 & 5213. & 0.263 & $0.21390 \times 10-3$ & 0.161 & 0.788 & 87.21 & 3.48 & 2.85 & $\mathrm{C}$ & 23.09 \\
\hline 205.3 & 5230. & 0.316 & $0.22074 \times 10-3$ & 0.161 & 0.788 & 87.21 & 2.94 & 2.85 & $\mathrm{C}$ & 20.35 \\
\hline 221.0 & 5261. & 0.410 & $0.23233 \times 10-3$ & 0.161 & 0.790 & 87.21 & 2.30 & 2.84 & $\mathrm{C}$ & 23.01 \\
\hline 239.0 & 5291. & 0.502 & $0.24332 \times 10-3$ & 0.161 & 0.791 & 87.21 & 1.90 & 2.83 & $\mathrm{C}$ & 26.76 \\
\hline 262.2 & 5323. & 0.603 & $0.25474 \times 10-3$ & 0.160 & 0.793 & 87.21 & 1.59 & 2.83 & $\mathrm{C}$ & 33.34 \\
\hline 295.3 & 5360. & 0.720 & $0.26737 \times 10-3$ & 0.160 & 0.794 & 87.21 & 1.34 & 2.82 & $\mathrm{C}$ & 44.49 \\
\hline 22.4 & 3119. & 0.006 & $0.30450 \times 10-4$ & 0.162 & 0.784 & 87.21 & 34.36 & 2.86 & $\mathrm{C}$ & 171.31 \\
\hline 41.0 & 3123. & 0.026 & $0.62140 \times 10-4$ & 0.162 & 0.785 & 87.21 & 20.29 & 2.86 & $\mathrm{C}$ & 27.57 \\
\hline 56.5 & 3129. & 0.057 & $0.91490 \times 10-4$ & 0.162 & 0.785 & 87.21 & 12.46 & 2.86 & $\mathrm{C}$ & 41.14 \\
\hline 70.5 & 3137. & 0.096 & $0.11857 \times 10-3$ & 0.162 & 0.786 & 87.21 & 8.38 & 2.86 & $\mathrm{C}$ & 32.33 \\
\hline 87.5 & 3148. & 0.153 & $0.14996 \times 10-3$ & 0.162 & 0.787 & 87.21 & 5.65 & 2.85 & $\mathrm{C}$ & 18.11 \\
\hline 97.7 & 3156. & 0.191 & $0.16745 \times 10-3$ & 0.162 & 0.787 & 87.21 & 4.66 & 2.85 & $\mathrm{C}$ & 33.70 \\
\hline 128.2 & 3175. & 0.287 & $0.21459 \times 10-3$ & 0.162 & 0.788 & 87.21 & 3.22 & 2.85 & $\mathrm{C}$ & 27.51 \\
\hline 131.2 & 3186. & 0.338 & $0.21372 \times 10-3$ & 0.162 & 0.789 & 87.21 & 2.76 & 2.84 & $\mathrm{C}$ & 21.89 \\
\hline 137.1 & 3205. & 0.427 & $0.21194 \times 10-3$ & 0.161 & 0.791 & 87.21 & 2.22 & 2.84 & $\mathrm{C}$ & 23.97 \\
\hline 143.9 & 3222. & 0.508 & $0.21039 \times 10-3$ & 0.161 & 0.792 & 87.21 & 1.87 & 2.83 & $\mathrm{C}$ & 28.33 \\
\hline 152.5 & 3239. & 0.593 & $0.20873 \times 10-3$ & 0.161 & 0.793 & 87.21 & 1.62 & 2.83 & $\mathrm{C}$ & 34.82 \\
\hline 164.4 & 3258. & 0.685 & $0.20688 \times 10-3$ & 0.161 & 0.794 & 87.21 & 1.41 & 2.82 & $\mathrm{C}$ & 48.69 \\
\hline 21.2 & 3048. & 0.006 & $0.30040 \times 10-4$ & 0.162 & 0.784 & 87.21 & 35.02 & 2.86 & $\mathrm{C}$ & 621.07 \\
\hline 39.7 & 3052. & 0.026 & $0.61980 \times 10-4$ & 0.162 & 0.785 & 87.21 & 20.57 & 2.86 & $\mathrm{C}$ & 87.64 \\
\hline 55.1 & 3058. & 0.056 & $0.91560 \times 10-4$ & 0.162 & 0.785 & 87.21 & 12.57 & 2.86 & $\mathrm{C}$ & 50.87 \\
\hline 69.1 & 3066. & 0.095 & $0.11884 \times 10-3$ & 0.162 & 0.786 & 87.21 & 8.43 & 2.86 & $\mathrm{C}$ & 54.87 \\
\hline 86.2 & 3077. & 0.153 & $0.15048 \times 10-3$ & 0.162 & 0.787 & 87.21 & 5.66 & 2.85 & $\mathrm{C}$ & 57.25 \\
\hline 96.4 & 3085. & 0.191 & $0.16811 \times 10-3$ & 0.162 & 0.787 & 87.21 & 4.66 & 2.85 & $\mathrm{C}$ & 57.21 \\
\hline
\end{tabular}




\begin{tabular}{|c|c|c|c|c|c|c|c|c|c|c|}
\hline 127.1 & 3103. & 0.287 & $0.21647 \times 10-3$ & 0.162 & 0.788 & 87.21 & 3.21 & 2.85 & $\mathrm{C}$ & 26.76 \\
\hline 130.4 & 3114. & 0.339 & $0.21590 \times 10-3$ & 0.162 & 0.789 & 87.21 & 2.75 & 2.84 & $\mathrm{C}$ & 21.81 \\
\hline 136.8 & 3133. & 0.428 & $0.21463 \times 10-3$ & 0.162 & 0.791 & 87.21 & 2.21 & 2.84 & $\mathrm{C}$ & 23.96 \\
\hline 144.0 & 3150. & 0.511 & $0.21355 \times 10-3$ & 0.161 & 0.792 & 87.21 & 1.86 & 2.83 & $\mathrm{C}$ & 28.35 \\
\hline 153.0 & 3167. & 0.597 & $0.21240 \times 10-3$ & 0.161 & 0.793 & 87.21 & 1.61 & 2.83 & $\mathrm{C}$ & 34.94 \\
\hline 165.5 & 3186. & 0.691 & $0.21110 \times 10-3$ & 0.161 & 0.794 & 87.21 & 1.39 & 2.82 & $\mathrm{C}$ & 48.47 \\
\hline 285.7 & 6667. & 0.147 & $0.57694 \times 10-3$ & 0.162 & 0.786 & 87.21 & 5.85 & 2.85 & $\mathrm{P}$ & 11.39 \\
\hline 297.5 & 6764. & 0.375 & $0.46451 \times 10-3$ & 0.162 & 0.790 & 87.21 & 2.50 & 2.84 & $\mathrm{P}$ & 14.76 \\
\hline 299.8 & 6836. & 0.543 & $0.35959 \times 10-3$ & 0.161 & 0.792 & 87.21 & 1.76 & 2.83 & $\mathrm{P}$ & 17.58 \\
\hline 284.7 & 6885. & 0.660 & $0.26162 \times 10-3$ & 0.161 & 0.794 & 87.21 & 1.46 & 2.82 & $\mathrm{P}$ & 24.52 \\
\hline 223.6 & 6921. & 0.749 & $0.14698 \times 10-3$ & 0.161 & 0.795 & 87.21 & 1.29 & 2.82 & $\mathrm{P}$ & 37.38 \\
\hline 153.1 & 6930. & 0.777 & $0.83190 \times 10-4$ & 0.160 & 0.795 & 87.21 & 1.24 & 2.82 & $\mathrm{P}$ & 39.75 \\
\hline 335.4 & 6916. & 0.826 & $0.13344 \times 10-3$ & 0.158 & 0.795 & 87.21 & 1.17 & 2.82 & $\mathrm{P}$ & 60.72 \\
\hline 389.7 & 6926. & 0.857 & $0.11507 \times 10-3$ & 0.158 & 0.795 & 87.21 & 1.13 & 2.82 & $\mathrm{P}$ & 56.88 \\
\hline 581.6 & 6937. & 0.899 & $0.83640 \times 10-4$ & 0.157 & 0.795 & 87.21 & 1.08 & 2.82 & $\mathrm{P}$ & 131.60 \\
\hline 1809.6 & 6942. & 0.926 & $0.54240 \times 10-4$ & 0.157 & 0.796 & 87.21 & 1.05 & 2.82 & $\mathrm{P}$ & 511.03 \\
\hline 287.9 & 9217. & 0.098 & $0.38832 \times 10-3$ & 0.163 & 0.786 & 87.21 & 8.22 & 2.86 & $\mathrm{P}$ & 12.71 \\
\hline 290.7 & 9304. & 0.252 & $0.31457 \times 10-3$ & 0.162 & 0.788 & 87.21 & 3.63 & 2.84 & $\mathrm{P}$ & 15.85 \\
\hline 284.0 & 9366. & 0.365 & $0.24591 \times 10-3$ & 0.162 & 0.790 & 87.21 & 2.56 & 2.84 & $\mathrm{P}$ & 18.03 \\
\hline 262.6 & 9408. & 0.446 & $0.18208 \times 10-3$ & 0.162 & 0.791 & 87.21 & 2.12 & 2.84 & $\mathrm{P}$ & 24.15 \\
\hline 204.4 & 9436. & 0.509 & $0.10765 \times 10-3$ & 0.161 & 0.791 & 87.21 & 1.87 & 2.83 & $\mathrm{P}$ & 35.66 \\
\hline 147.3 & 9442. & 0.530 & $0.66290 \times 10-4$ & 0.161 & 0.792 & 87.21 & 1.80 & 2.83 & $\mathrm{P}$ & 37.32 \\
\hline 291.7 & 9399. & 0.572 & $0.10760 \times 10-3$ & 0.158 & 0.791 & 87.21 & 1.68 & 2.84 & $\mathrm{P}$ & 50.80 \\
\hline 313.4 & 9405. & 0.597 & $0.93690 \times 10-4$ & 0.157 & 0.791 & 87.21 & 1.61 & 2.83 & $\mathrm{P}$ & 43.43 \\
\hline 362.6 & 9410. & 0.632 & $0.69980 \times 10-4$ & 0.157 & 0.791 & 87.21 & 1.52 & 2.84 & $\mathrm{P}$ & 87.72 \\
\hline 433.0 & 9408. & 0.655 & $0.47820 \times 10-4$ & 0.156 & 0.791 & 87.21 & 1.47 & 2.84 & $\mathrm{P}$ & 119.80 \\
\hline 606.5 & 9398. & 0.671 & $0.24820 \times 10-4$ & 0.155 & 0.791 & 87.21 & 1.44 & 2.84 & $\mathrm{P}$ & 1035.55 \\
\hline 282.9 & 9208. & 0.097 & $0.38314 \times 10-3$ & 0.162 & 0.786 & 87.21 & 8.28 & 2.86 & $\mathrm{P}$ & 12.62 \\
\hline 284.5 & 9293. & 0.249 & $0.30982 \times 10-3$ & 0.162 & 0.788 & 87.21 & 3.66 & 2.85 & $\mathrm{P}$ & 15.94 \\
\hline 275.8 & 9354. & 0.361 & $0.24156 \times 10-3$ & 0.161 & 0.789 & 87.21 & 2.60 & 2.84 & $\mathrm{P}$ & 17.98 \\
\hline 252.6 & 9395. & 0.439 & $0.17810 \times 10-3$ & 0.161 & 0.790 & 87.21 & 2.15 & 2.84 & $\mathrm{P}$ & 24.13 \\
\hline 192.7 & 9422. & 0.501 & $0.10412 \times 10-3$ & 0.160 & 0.791 & 87.21 & 1.90 & 2.84 & $\mathrm{P}$ & 35.19 \\
\hline 135.7 & 9427. & 0.521 & $0.63020 \times 10-4$ & 0.160 & 0.791 & 87.21 & 1.83 & 2.83 & $\mathrm{P}$ & 36.71 \\
\hline 271.5 & 9383. & 0.562 & $0.10426 \times 10-3$ & 0.157 & 0.790 & 87.21 & 1.71 & 2.84 & $\mathrm{P}$ & 50.12 \\
\hline 288.8 & 9388. & 0.586 & $0.90750 \times 10-4$ & 0.157 & 0.790 & 87.21 & 1.64 & 2.84 & $\mathrm{P}$ & 42.30 \\
\hline 323.7 & 9392. & 0.620 & $0.67720 \times 10-4$ & 0.156 & 0.790 & 87.21 & 1.55 & 2.84 & $\mathrm{P}$ & 85.01 \\
\hline 364.7 & 9389. & 0.642 & $0.46200 \times 10-4$ & 0.155 & 0.790 & 87.21 & 1.50 & 2.84 & $\mathrm{P}$ & 108.60 \\
\hline 418.5 & 9379. & 0.657 & $0.23870 \times 10-4$ & 0.154 & 0.790 & 87.21 & 1.47 & 2.84 & $\mathrm{P}$ & 1190.65 \\
\hline 244.9 & 5211. & 0.146 & $0.57268 \times 10-3$ & 0.161 & 0.786 & 87.21 & 5.91 & 2.86 & $\mathrm{P}$ & 12.03 \\
\hline 255.8 & 5287. & 0.371 & $0.45687 \times 10-3$ & 0.161 & 0.789 & 87.21 & 2.53 & 2.84 & $\mathrm{P}$ & 17.10 \\
\hline 257.6 & 5343. & 0.535 & $0.34879 \times 10-3$ & 0.160 & 0.792 & 87.21 & 1.79 & 2.83 & $\mathrm{P}$ & 21.45 \\
\hline 242.0 & 5382. & 0.647 & $0.24789 \times 10-3$ & 0.160 & 0.793 & 87.21 & 1.49 & 2.83 & $\mathrm{P}$ & 30.80 \\
\hline 179.1 & 5409. & 0.729 & $0.12989 \times 10-3$ & 0.160 & 0.794 & 87.21 & 1.32 & 2.82 & $\mathrm{P}$ & 47.29 \\
\hline 107.2 & 5416. & 0.753 & $0.64230 \times 10-4$ & 0.160 & 0.795 & 87.21 & 1.28 & 2.82 & $\mathrm{P}$ & 49.62 \\
\hline 294.2 & 5413. & 0.794 & $0.12495 \times 10-3$ & 0.159 & 0.794 & 87.21 & 1.22 & 2.82 & $\mathrm{P}$ & 82.54 \\
\hline 343.0 & 5421. & 0.822 & $0.10655 \times 10-3$ & 0.158 & 0.795 & 87.21 & 1.18 & 2.82 & $\mathrm{P}$ & 69.52 \\
\hline 517.0 & 5432. & 0.861 & $0.74990 \times 10-4$ & 0.158 & 0.795 & 87.21 & 1.13 & 2.82 & $\mathrm{P}$ & 174.26 \\
\hline 1662.1 & 5436. & 0.884 & $0.45470 \times 10-4$ & 0.158 & 0.795 & 87.21 & 1.10 & 2.82 & $\mathrm{P}$ & 660.28 \\
\hline 174.9 & 3245. & 0.139 & $0.54335 \times 10-3$ & 0.162 & 0.786 & 87.21 & 6.17 & 2.85 & $\mathrm{P}$ & 16.03 \\
\hline
\end{tabular}




\begin{tabular}{|c|c|c|c|c|c|c|c|c|c|c|}
\hline 187.0 & 3290. & 0.352 & $0.43320 \times 10-3$ & 0.162 & 0.789 & 87.21 & 2.66 & 2.84 & $\mathrm{P}$ & 24.70 \\
\hline 194.1 & 3324. & 0.507 & $0.33048 \times 10-3$ & 0.162 & 0.792 & 87.21 & 1.88 & 2.83 & $\mathrm{P}$ & 31.45 \\
\hline 189.6 & 3348. & 0.613 & $0.23465 \times 10-3$ & 0.161 & 0.793 & 87.21 & 1.57 & 2.83 & $\mathrm{P}$ & 45.97 \\
\hline 148.4 & 3365. & 0.691 & $0.12264 \times 10-3$ & 0.161 & 0.794 & 87.21 & 1.39 & 2.82 & $\mathrm{P}$ & 77.93 \\
\hline 91.6 & 3370. & 0.713 & $0.60320 \times 10-4$ & 0.161 & 0.795 & 87.21 & 1.35 & 2.82 & $\mathrm{P}$ & 81.15 \\
\hline 307.5 & 3374. & 0.751 & $0.12114 \times 10-3$ & 0.161 & 0.795 & 87.21 & 1.29 & 2.82 & $\mathrm{P}$ & 191.93 \\
\hline 413.2 & 3380. & 0.779 & $0.10026 \times 10-3$ & 0.161 & 0.796 & 87.21 & 1.24 & 2.82 & $\mathrm{P}$ & 124.01 \\
\hline 1866.1 & 3388. & 0.814 & $0.64340 \times 10-4$ & 0.161 & 0.796 & 87.21 & 1.19 & 2.82 & $\mathrm{P}$ & 794.01 \\
\hline 176.4 & 3288. & 0.141 & $0.55349 \times 10-3$ & 0.162 & 0.787 & 87.21 & 6.06 & 2.85 & $\mathrm{P}$ & 15.62 \\
\hline 188.1 & 3335. & 0.359 & $0.44079 \times 10-3$ & 0.162 & 0.790 & 87.21 & 2.61 & 2.84 & $\mathrm{P}$ & 24.06 \\
\hline 194.6 & 3370. & 0.517 & $0.33567 \times 10-3$ & 0.162 & 0.792 & 87.21 & 1.84 & 2.83 & $\mathrm{P}$ & 30.48 \\
\hline 188.9 & 3394. & 0.624 & $0.23759 \times 10-3$ & 0.162 & 0.794 & 87.21 & 1.54 & 2.83 & $\mathrm{P}$ & 45.09 \\
\hline 145.3 & 3412. & 0.702 & $0.12291 \times 10-3$ & 0.162 & 0.795 & 87.21 & 1.37 & 2.82 & $\mathrm{P}$ & 77.08 \\
\hline 87.2 & 3417. & 0.724 & $0.59120 \times 10-4$ & 0.162 & 0.795 & 87.21 & 1.33 & 2.82 & $\mathrm{P}$ & 80.03 \\
\hline 291.5 & 3421. & 0.763 & $0.12072 \times 10-3$ & 0.161 & 0.796 & 87.21 & 1.27 & 2.82 & $\mathrm{P}$ & 186.40 \\
\hline 379.7 & 3427. & 0.790 & $0.99880 \times 10-4$ & 0.161 & 0.796 & 87.21 & 1.22 & 2.81 & $\mathrm{P}$ & 117.13 \\
\hline 1150.4 & 3434. & 0.825 & $0.64060 \times 10-4$ & 0.161 & 0.797 & 87.21 & 1.17 & 2.81 & $\mathrm{P}$ & 543.88 \\
\hline 234.2 & 5226. & 0.141 & $0.55570 \times 10-3$ & 0.162 & 0.786 & 87.21 & 6.07 & 2.86 & $\mathrm{P}$ & 12.12 \\
\hline 245.0 & 5300. & 0.361 & $0.44720 \times 10-3$ & 0.161 & 0.789 & 87.21 & 2.60 & 2.84 & $\mathrm{P}$ & 16.79 \\
\hline 248.4 & 5355. & 0.522 & $0.34598 \times 10-3$ & 0.161 & 0.792 & 87.21 & 1.83 & 2.83 & $\mathrm{P}$ & 20.32 \\
\hline 237.6 & 5394. & 0.634 & $0.25151 \times 10-3$ & 0.161 & 0.793 & 87.21 & 1.52 & 2.83 & $\mathrm{P}$ & 29.12 \\
\hline 187.8 & 5422. & 0.720 & $0.14100 \times 10-3$ & 0.161 & 0.795 & 87.21 & 1.34 & 2.82 & $\mathrm{P}$ & 45.39 \\
\hline 128.5 & 5430. & 0.746 & $0.79520 \times 10-4$ & 0.161 & 0.795 & 87.21 & 1.29 & 2.82 & $\mathrm{P}$ & 47.70 \\
\hline 292.5 & 5428. & 0.792 & $0.12639 \times 10-3$ & 0.159 & 0.795 & 87.21 & 1.22 & 2.82 & $\mathrm{P}$ & 81.67 \\
\hline 343.5 & 5437. & 0.821 & $0.10793 \times 10-3$ & 0.159 & 0.795 & 87.21 & 1.18 & 2.82 & $\mathrm{P}$ & 68.70 \\
\hline 533.2 & 5447. & 0.860 & $0.76250 \times 10-4$ & 0.159 & 0.796 & 87.21 & 1.13 & 2.82 & $\mathrm{P}$ & 173.89 \\
\hline 2419.3 & 5452. & 0.884 & $0.46630 \times 10-4$ & 0.158 & 0.796 & 87.21 & 1.10 & 2.82 & $\mathrm{P}$ & 923.34 \\
\hline 90.8 & 6530. & 0.010 & $0.50520 \times 10-4$ & 0.162 & 0.785 & 87.21 & 30.50 & 2.86 & $\mathrm{C}$ & 52.83 \\
\hline 96.0 & 6540. & 0.035 & $0.64660 \times 10-4$ & 0.162 & 0.785 & 87.21 & 17.26 & 2.86 & $\mathrm{C}$ & 35.27 \\
\hline 101.1 & 6551. & 0.063 & $0.77760 \times 10-4$ & 0.162 & 0.785 & 87.21 & 11.53 & 2.86 & $\mathrm{C}$ & 27.74 \\
\hline 106.3 & 6564. & 0.095 & $0.89830 \times 10-4$ & 0.162 & 0.786 & 87.21 & 8.48 & 2.86 & $\mathrm{C}$ & 22.09 \\
\hline 113.0 & 6581. & 0.136 & $0.10382 \times 10-3$ & 0.162 & 0.786 & 87.21 & 6.26 & 2.85 & $\mathrm{C}$ & 18.98 \\
\hline 117.0 & 6592. & 0.162 & $0.11160 \times 10-3$ & 0.162 & 0.787 & 87.21 & 5.40 & 2.85 & $\mathrm{C}$ & 17.41 \\
\hline 215.6 & 6618. & 0.242 & $0.21747 \times 10-3$ & 0.161 & 0.788 & 87.21 & 3.75 & 2.85 & $\mathrm{C}$ & 19.65 \\
\hline 226.2 & 6641. & 0.296 & $0.22815 \times 10-3$ & 0.161 & 0.788 & 87.21 & 3.12 & 2.85 & $\mathrm{C}$ & 18.29 \\
\hline 246.3 & 6680. & 0.395 & $0.24637 \times 10-3$ & 0.161 & 0.790 & 87.21 & 2.38 & 2.84 & $\mathrm{C}$ & 21.08 \\
\hline 268.2 & 6719. & 0.494 & $0.26357 \times 10-3$ & 0.161 & 0.791 & 87.21 & 1.92 & 2.84 & $\mathrm{C}$ & 24.63 \\
\hline 295.2 & 6761. & 0.605 & $0.28147 \times 10-3$ & 0.160 & 0.792 & 87.21 & 1.59 & 2.83 & $\mathrm{C}$ & 30.83 \\
\hline 331.9 & 6810. & 0.735 & $0.30128 \times 10-3$ & 0.159 & 0.794 & 87.21 & 1.31 & 2.82 & $\mathrm{C}$ & 40.40 \\
\hline 80.4 & 6650. & 0.006 & $0.47730 \times 10-4$ & 0.163 & 0.785 & 87.21 & 33.72 & 2.86 & $\mathrm{C}$ & 48.81 \\
\hline 88.4 & 6659. & 0.030 & $0.61350 \times 10-4$ & 0.163 & 0.785 & 87.21 & 18.70 & 2.86 & $\mathrm{C}$ & 33.51 \\
\hline 95.4 & 6670. & 0.057 & $0.73960 \times 10-4$ & 0.163 & 0.786 & 87.21 & 12.37 & 2.86 & $\mathrm{C}$ & 27.17 \\
\hline 101.7 & 6683. & 0.087 & $0.85600 \times 10-4$ & 0.163 & 0.786 & 87.21 & 9.05 & 2.86 & $\mathrm{C}$ & 21.84 \\
\hline 109.2 & 6700. & 0.127 & $0.99070 \times 10-4$ & 0.163 & 0.787 & 87.21 & 6.65 & 2.85 & $\mathrm{C}$ & 18.81 \\
\hline 113.4 & 6710. & 0.151 & $0.10657 \times 10-3$ & 0.163 & 0.787 & 87.21 & 5.72 & 2.85 & $\mathrm{C}$ & 17.36 \\
\hline 210.6 & 6735. & 0.228 & $0.20819 \times 10-3$ & 0.162 & 0.788 & 87.21 & 3.96 & 2.85 & $\mathrm{C}$ & 19.64 \\
\hline 220.7 & 6757. & 0.280 & $0.21846 \times 10-3$ & 0.162 & 0.789 & 87.21 & 3.29 & 2.85 & $\mathrm{C}$ & 18.21 \\
\hline 239.6 & 6795. & 0.374 & $0.23598 \times 10-3$ & 0.162 & 0.790 & 87.21 & 2.51 & 2.84 & $\mathrm{C}$ & 20.94 \\
\hline 259.8 & 6833. & 0.470 & $0.25251 \times 10-3$ & 0.161 & 0.791 & 87.21 & 2.02 & 2.83 & $\mathrm{C}$ & 24.34 \\
\hline
\end{tabular}




\begin{tabular}{|c|l|l|l|l|l|l|l|l|l|l|}
\hline 283.9 & 6874. & 0.575 & $0.26971 \times 10-3$ & 0.161 & 0.792 & 87.21 & 1.66 & 2.83 & $\mathrm{C}$ & 30.21 \\
\hline 315.8 & 6921. & 0.700 & $0.28873 \times 10-3$ & 0.160 & 0.794 & 87.21 & 1.38 & 2.82 & $\mathrm{C}$ & 39.16 \\
\hline 102.6 & 6650. & 0.009 & $0.50550 \times 10-4$ & 0.162 & 0.784 & 87.21 & 30.92 & 2.86 & $\mathrm{C}$ & 58.22 \\
\hline 108.3 & 6661. & 0.035 & $0.66360 \times 10-4$ & 0.162 & 0.785 & 87.21 & 17.28 & 2.86 & $\mathrm{C}$ & 38.29 \\
\hline 113.9 & 6673. & 0.064 & $0.81010 \times 10-4$ & 0.162 & 0.785 & 87.21 & 11.41 & 2.86 & $\mathrm{C}$ & 28.60 \\
\hline 119.6 & 6687. & 0.097 & $0.94520 \times 10-4$ & 0.162 & 0.786 & 87.21 & 8.31 & 2.86 & $\mathrm{C}$ & 23.64 \\
\hline 126.7 & 6705. & 0.141 & $0.11017 \times 10-3$ & 0.162 & 0.786 & 87.21 & 6.08 & 2.86 & $\mathrm{C}$ & 18.96 \\
\hline 131.1 & 6717. & 0.168 & $0.11888 \times 10-3$ & 0.162 & 0.787 & 87.21 & 5.21 & 2.85 & $\mathrm{C}$ & 18.32 \\
\hline 213.0 & 6743. & 0.248 & $0.20730 \times 10-3$ & 0.161 & 0.787 & 87.21 & 3.67 & 2.85 & $\mathrm{C}$ & 20.33 \\
\hline 222.3 & 6765. & 0.300 & $0.21842 \times 10-3$ & 0.161 & 0.788 & 87.21 & 3.09 & 2.85 & $\mathrm{C}$ & 18.61 \\
\hline 239.8 & 6803. & 0.395 & $0.23737 \times 10-3$ & 0.161 & 0.789 & 87.21 & 2.39 & 2.84 & $\mathrm{C}$ & 21.02 \\
\hline 258.5 & 6841. & 0.491 & $0.25526 \times 10-3$ & 0.160 & 0.791 & 87.21 & 1.94 & 2.83 & $\mathrm{C}$ & 24.01 \\
\hline 281.2 & 6883. & 0.598 & $0.27387 \times 10-3$ & 0.160 & 0.792 & 87.21 & 1.60 & 2.83 & $\mathrm{C}$ & 29.55 \\
\hline 310.4 & 6931. & 0.725 & $0.29445 \times 10-3$ & 0.159 & 0.794 & 87.21 & 1.33 & 2.82 & $\mathrm{C}$ & 37.89 \\
\hline 94.2 & 6701. & 0.007 & $0.50300 \times 10-4$ & 0.162 & 0.784 & 87.21 & 33.66 & 2.86 & $\mathrm{C}$ & 53.76 \\
\hline 103.4 & 6711. & 0.032 & $0.66350 \times 10-4$ & 0.162 & 0.785 & 87.21 & 18.12 & 2.86 & $\mathrm{C}$ & 36.54 \\
\hline 111.2 & 6723. & 0.062 & $0.81220 \times 10-4$ & 0.162 & 0.785 & 87.21 & 11.76 & 2.86 & $\mathrm{C}$ & 27.83 \\
\hline 118.4 & 6737. & 0.094 & $0.94940 \times 10-4$ & 0.162 & 0.786 & 87.21 & 8.49 & 2.86 & $\mathrm{C}$ & 23.29 \\
\hline 126.8 & 6756. & 0.139 & $0.11082 \times 10-3$ & 0.162 & 0.786 & 87.21 & 6.17 & 2.85 & $\mathrm{C}$ & 18.78 \\
\hline 131.9 & 6768. & 0.166 & $0.11966 \times 10-3$ & 0.162 & 0.787 & 87.21 & 5.27 & 2.85 & $\mathrm{C}$ & 18.25 \\
\hline 216.6 & 6794. & 0.247 & $0.20964 \times 10-3$ & 0.161 & 0.788 & 87.21 & 3.69 & 2.85 & $\mathrm{C}$ & 20.17 \\
\hline 227.0 & 6816. & 0.299 & $0.22123 \times 10-3$ & 0.161 & 0.788 & 87.21 & 3.09 & 2.85 & $\mathrm{C}$ & 18.64 \\
\hline 246.1 & 6856. & 0.395 & $0.24102 \times 10-3$ & 0.161 & 0.790 & 87.21 & 2.38 & 2.84 & $\mathrm{C}$ & 21.10 \\
\hline 266.0 & 6894. & 0.493 & $0.25968 \times 10-3$ & 0.161 & 0.791 & 87.21 & 1.93 & 2.84 & $\mathrm{C}$ & 24.17 \\
\hline 289.6 & 6937. & 0.602 & $0.27911 \times 10-3$ & 0.160 & 0.792 & 87.21 & 1.59 & 2.83 & $\mathrm{C}$ & 29.77 \\
\hline 320.4 & 6987. & 0.732 & $0.30061 \times 10-3$ & 0.159 & 0.794 & 87.21 & 1.32 & 2.82 & $\mathrm{C}$ & 38.12 \\
\hline
\end{tabular}




\section{APPENDIX A3}

Convective Boiling of R452B within a micro-fin tube

(file: TaevapGWP3.dat)

\begin{tabular}{|c|c|c|c|c|c|c|c|c|c|c|}
\hline $\mathrm{Nu}$ & $\mathrm{Re}$ & $x_{\mathrm{q}}$ & Bo & $P_{\mathrm{s}} / P_{\mathrm{c}}$ & $T_{\mathrm{s}} / T_{\mathrm{c}}$ & $M_{\mathrm{w}}$ & $S v$ & $\operatorname{Pr}$ & flow & $U_{\mathrm{Nu}}$ \\
\hline 186.8 & 7806. & 0.006 & $0.18930 \times 10-4$ & 0.160 & 0.786 & 63.52 & 30.41 & 2.04 & $\mathrm{C}$ & 972.46 \\
\hline 166.6 & 7806. & 0.018 & $0.32310 \times 10-4$ & 0.160 & 0.786 & 63.52 & 22.56 & 2.04 & $\mathrm{C}$ & 98.98 \\
\hline 161.3 & 7807. & 0.033 & $0.44700 \times 10-4$ & 0.160 & 0.786 & 63.52 & 16.60 & 2.04 & $\mathrm{C}$ & 65.34 \\
\hline 159.9 & 7808. & 0.053 & $0.56120 \times 10-4$ & 0.160 & 0.786 & 63.52 & 12.59 & 2.04 & $\mathrm{C}$ & 48.15 \\
\hline 159.8 & 7809. & 0.080 & $0.69360 \times 10-4$ & 0.159 & 0.786 & 63.52 & 9.34 & 2.05 & $\mathrm{C}$ & 33.83 \\
\hline 160.2 & 7810. & 0.098 & $0.76730 \times 10-4$ & 0.159 & 0.786 & 63.52 & 8.01 & 2.05 & $\mathrm{C}$ & 35.15 \\
\hline 247.3 & 7808. & 0.152 & $0.14273 \times 10-3$ & 0.159 & 0.786 & 63.52 & 5.59 & 2.05 & $\mathrm{C}$ & 30.30 \\
\hline 259.4 & 7810. & 0.190 & $0.16027 \times 10-3$ & 0.159 & 0.786 & 63.52 & 4.62 & 2.06 & $\mathrm{C}$ & 26.46 \\
\hline 277.1 & 7814. & 0.265 & $0.19036 \times 10-3$ & 0.159 & 0.786 & 63.52 & 3.43 & 2.06 & $\mathrm{C}$ & 25.33 \\
\hline 291.9 & 7817. & 0.347 & $0.21880 \times 10-3$ & 0.159 & 0.786 & 63.52 & 2.68 & 2.07 & $\mathrm{C}$ & 25.98 \\
\hline 305.9 & 7820. & 0.445 & $0.24861 \times 10-3$ & 0.158 & 0.786 & 63.52 & 2.12 & 2.08 & $\mathrm{C}$ & 28.21 \\
\hline 321.1 & 7824. & 0.568 & $0.28196 \times 10-3$ & 0.158 & 0.787 & 63.52 & 1.68 & 2.10 & $\mathrm{C}$ & 31.73 \\
\hline 104.8 & 10993. & 0.004 & $0.875 \times 10-5$ & 0.162 & 0.787 & 63.52 & 32.32 & 2.04 & $\mathrm{C}$ & 275.74 \\
\hline 126.9 & 10993. & 0.010 & $0.17900 \times 10-4$ & 0.162 & 0.787 & 63.52 & 27.09 & 2.04 & $\mathrm{C}$ & 93.17 \\
\hline 136.3 & 10993. & 0.019 & $0.26380 \times 10-4$ & 0.162 & 0.787 & 63.52 & 21.70 & 2.04 & $\mathrm{C}$ & 66.92 \\
\hline 141.8 & 10993. & 0.030 & $0.34180 \times 10-4$ & 0.162 & 0.787 & 63.52 & 17.36 & 2.04 & $\mathrm{C}$ & 50.22 \\
\hline 146.5 & 10994. & 0.047 & $0.43210 \times 10-4$ & 0.162 & 0.787 & 63.52 & 13.40 & 2.04 & $\mathrm{C}$ & 34.72 \\
\hline 148.5 & 10995. & 0.059 & $0.48230 \times 10-4$ & 0.162 & 0.787 & 63.52 & 11.65 & 2.04 & $\mathrm{C}$ & 38.77 \\
\hline 238.9 & 10986. & 0.094 & $0.94470 \times 10-4$ & 0.161 & 0.787 & 63.52 & 8.27 & 2.04 & $\mathrm{C}$ & 29.11 \\
\hline 253.8 & 10987. & 0.119 & $0.10766 \times 10-3$ & .161 & 0.787 & 63.52 & 6.85 & 2.05 & $\mathrm{C}$ & 26.47 \\
\hline 274.7 & 10987. & 0.170 & $0.13030 \times 10-3$ & 0.161 & 0.787 & 63.52 & 5.08 & 2.05 & $\mathrm{C}$ & 25.06 \\
\hline 289.9 & 10986. & 0.226 & $0.15156 \times 10-3$ & 0.161 & 0.787 & 63.52 & 3.95 & 2.06 & $\mathrm{C}$ & 25.56 \\
\hline 303.0 & 10983. & 0.294 & $0.17373 \times 10-3$ & 0.160 & 0.787 & 63.52 & 3.12 & 2.06 & $\mathrm{C}$ & 27.28 \\
\hline 314.9 & 10979. & 0.379 & $0.19831 \times 10-3$ & 0.160 & 0.787 & 63.52 & 2.46 & 2.07 & $\mathrm{C}$ & 29.95 \\
\hline 71.5 & 5727. & 0.003 & $0.16450 \times 10-4$ & 0.164 & 0.788 & 63.52 & 32.88 & 2.04 & $\mathrm{C}$ & 245.99 \\
\hline 102.8 & 5727. & 0.014 & $0.32920 \times 10-4$ & 0.164 & 0.788 & 63.52 & 24.15 & 2.04 & $\mathrm{C}$ & 76.24 \\
\hline 120.3 & 5728. & 0.031 & $0.48160 \times 10-4$ & 0.164 & 0.788 & 63.52 & 17.19 & 2.04 & $\mathrm{C}$ & 62.06 \\
\hline 131.7 & 5729. & 0.052 & $0.62220 \times 10-4$ & 0.164 & 0.788 & 63.52 & 12.63 & 2.04 & $\mathrm{C}$ & 48.55 \\
\hline 142.1 & 5730. & 0.083 & $0.78520 \times 10-4$ & 0.164 & 0.788 & 63.52 & 9.08 & 2.04 & $\mathrm{C}$ & 34.97 \\
\hline 147.0 & 5731. & 0.103 & $0.87590 \times 10-4$ & 0.164 & 0.789 & 63.52 & 7.67 & 2.04 & $\mathrm{C}$ & 39.32 \\
\hline 232.3 & 5731. & 0.164 & $0.15975 \times 10-3$ & 0.163 & 0.789 & 63.52 & 5.22 & 2.05 & $\mathrm{C}$ & 33.10 \\
\hline 243.9 & 5733. & 0.206 & $0.17709 \times 10-3$ & 0.163 & 0.789 & 63.52 & 4.28 & 2.05 & $\mathrm{C}$ & 28.81 \\
\hline 262.3 & 5737. & 0.288 & $0.20679 \times 10-3$ & 0.163 & 0.789 & 63.52 & 3.17 & 2.06 & $\mathrm{C}$ & 28.53 \\
\hline 278.6 & 5740. & 0.377 & $0.23496 \times 10-3$ & 0.163 & 0.789 & 63.52 & 2.47 & 2.07 & $\mathrm{C}$ & 29.07 \\
\hline 295.6 & 5745. & 0.481 & $0.26455 \times 10-3$ & 0.163 & 0.789 & 63.52 & 1.96 & 2.08 & $\mathrm{C}$ & 31.62 \\
\hline 315.9 & 5753. & 0.612 & $0.29776 \times 10-3$ & 0.162 & 0.789 & 63.52 & 1.56 & 2.10 & $\mathrm{C}$ & 36.03 \\
\hline 46.0 & 3932. & 0.003 & $0.15660 \times 10-4$ & 0.163 & 0.788 & 63.52 & 33.31 & 2.04 & $\mathrm{C}$ & 2644.68 \\
\hline 77.4 & 3932. & 0.014 & $0.33460 \times 10-4$ & 0.163 & 0.788 & 63.52 & 24.40 & 2.04 & $\mathrm{C}$ & 167.55 \\
\hline 97.3 & 3933. & 0.031 & $0.49940 \times 10-4$ & 0.163 & 0.788 & 63.52 & 17.19 & 2.04 & $\mathrm{C}$ & 91.04 \\
\hline 111.6 & 3933. & 0.053 & $0.65150 \times 10-4$ & 0.163 & 0.788 & 63.52 & 12.49 & 2.04 & $\mathrm{C}$ & 97.74 \\
\hline 125.4 & 3934. & 0.085 & $0.82780 \times 10-4$ & 0.162 & 0.788 & 63.52 & 8.88 & 2.04 & $\mathrm{C}$ & 104.12 \\
\hline
\end{tabular}




\begin{tabular}{|c|c|c|c|c|c|c|c|c|c|c|}
\hline 132.2 & 3935. & 0.107 & $0.92590 \times 10-4$ & 0.162 & 0.788 & 63.52 & 7.46 & 2.05 & $\mathrm{C}$ & 103.22 \\
\hline 185.5 & 3936. & 0.166 & $0.14554 \times 10-3$ & 0.162 & 0.788 & 63.52 & 5.18 & 2.05 & $\mathrm{C}$ & 37.65 \\
\hline 198.7 & 3937. & 0.204 & $0.16228 \times 10-3$ & 0.162 & 0.788 & 63.52 & 4.32 & 2.05 & $\mathrm{C}$ & 34.33 \\
\hline 220.7 & 3940. & 0.279 & $0.19094 \times 10-3$ & 0.162 & 0.788 & 63.52 & 3.26 & 2.06 & $\mathrm{C}$ & 33.55 \\
\hline 240.7 & 3943. & 0.361 & $0.21809 \times 10-3$ & 0.162 & 0.788 & 63.52 & 2.57 & 2.07 & $\mathrm{C}$ & 34.91 \\
\hline 262.4 & 3947. & 0.458 & $0.24659 \times 10-3$ & 0.162 & 0.789 & 63.52 & 2.06 & 2.08 & $\mathrm{C}$ & 37.66 \\
\hline 288.8 & 3952. & 0.580 & $0.27851 \times 10-3$ & 0.162 & 0.789 & 63.52 & 1.64 & 2.09 & $\mathrm{C}$ & 42.90 \\
\hline 312.3 & 8389. & 0.090 & $0.34169 \times 10-3$ & 0.163 & 0.788 & 63.52 & 8.50 & 2.04 & $\mathrm{P}$ & 19.85 \\
\hline 299.5 & 8398. & 0.230 & $0.28173 \times 10-3$ & 0.163 & 0.788 & 63.52 & 3.88 & 2.06 & $\mathrm{P}$ & 22.79 \\
\hline 279.4 & 8405. & 0.336 & $0.22588 \times 10-3$ & 0.162 & 0.788 & 63.52 & 2.75 & 2.06 & $\mathrm{P}$ & 24.85 \\
\hline 250.6 & 8409. & 0.414 & $0.17372 \times 10-3$ & 0.162 & 0.789 & 63.52 & 2.26 & 2.07 & $\mathrm{P}$ & 30.49 \\
\hline 197.2 & 8412. & 0.478 & $0.11255 \times 10-3$ & 0.162 & 0.789 & 63.52 & 1.98 & 2.08 & $\mathrm{P}$ & 43.00 \\
\hline 154.4 & 8412. & 0.502 & $0.78480 \times 10-4$ & 0.162 & 0.789 & 63.52 & 1.89 & 2.08 & $\mathrm{P}$ & 44.33 \\
\hline 285.4 & 8388. & 0.552 & $0.12158 \times 10-3$ & 0.160 & 0.788 & 63.52 & 1.73 & 2.09 & $\mathrm{P}$ & 61.68 \\
\hline 290.8 & 8388. & 0.581 & $0.10635 \times 10-3$ & 0.160 & 0.788 & 63.52 & 1.64 & 2.10 & $\mathrm{P}$ & 46.22 \\
\hline 298.2 & 8386. & 0.622 & $0.80080 \times 10-4$ & 0.160 & 0.788 & 63.52 & 1.54 & 2.10 & $\mathrm{P}$ & 93.51 \\
\hline 299.2 & 8383. & 0.650 & $0.55460 \times 10-4$ & 0.159 & 0.788 & 63.52 & 1.48 & 2.10 & $\mathrm{P}$ & 103.31 \\
\hline 279.5 & 8377. & 0.668 & $0.29790 \times 10-4$ & 0.159 & 0.788 & 63.52 & 1.44 & 2.11 & $\mathrm{P}$ & 774.88 \\
\hline 50.2 & 8367. & 0.676 & $0.146 \times 10-5$ & 0.158 & 0.787 & 63.52 & 1.42 & 2.11 & $\mathrm{P}$ & 989.45 \\
\hline 312.0 & 11040. & 0.069 & $0.25705 \times 10-3$ & 0.164 & 0.789 & 63.52 & 10.40 & 2.04 & $\mathrm{P}$ & 20.29 \\
\hline 296.5 & 11046. & 0.174 & $0.21190 \times 10-3$ & 0.164 & 0.789 & 63.52 & 4.96 & 2.05 & $\mathrm{P}$ & 22.98 \\
\hline 273.9 & 11050. & 0.253 & $0.16990 \times 10-3$ & 0.164 & 0.789 & 63.52 & 3.56 & 2.06 & $\mathrm{P}$ & 25.04 \\
\hline 243.0 & 11052. & 0.311 & $0.13081 \times 10-3$ & 0.163 & 0.789 & 63.52 & 2.95 & 2.06 & $\mathrm{P}$ & 30.54 \\
\hline 189.4 & 11050. & 0.360 & $0.85140 \times 10-4$ & 0.163 & 0.789 & 63.52 & 2.58 & 2.07 & $\mathrm{P}$ & 42.60 \\
\hline 148.0 & 11048. & 0.378 & $0.59740 \times 10-4$ & 0.163 & 0.789 & 63.52 & 2.47 & 2.07 & $\mathrm{P}$ & 43.77 \\
\hline 273.1 & 11003. & 0.417 & $0.96740 \times 10-4$ & 0.161 & 0.788 & 63.52 & 2.25 & 2.07 & $\mathrm{P}$ & 58.83 \\
\hline 272.7 & 11001. & 0.440 & $0.84590 \times 10-4$ & 0.161 & 0.788 & 63.52 & 2.14 & 2.08 & $\mathrm{P}$ & 42.54 \\
\hline 266.3 & 10994. & 0.473 & $0.63730 \times 10-4$ & 0.160 & 0.788 & 63.52 & 2.00 & 2.08 & $\mathrm{P}$ & 83.40 \\
\hline 247.7 & 10985. & 0.495 & $0.44200 \times 10-4$ & 0.160 & 0.787 & 63.52 & 1.91 & 2.09 & $\mathrm{P}$ & 89.04 \\
\hline 198.2 & 10973. & 0.510 & $0.23890 \times 10-4$ & 0.159 & 0.787 & 63.52 & 1.86 & 2.09 & $\mathrm{P}$ & 774.44 \\
\hline 23.6 & 10957. & 0.516 & $0.148 \times 10-5$ & 0.159 & 0.787 & 63.52 & 1.84 & 2.09 & $\mathrm{P}$ & 800.63 \\
\hline 290.7 & 6173. & 0.099 & $0.37370 \times 10-3$ & 0.165 & 0.789 & 63.52 & 7.88 & 2.04 & $\mathrm{P}$ & 21.23 \\
\hline 278.9 & 6181. & 0.252 & $0.30709 \times 10-3$ & 0.165 & 0.790 & 63.52 & 3.57 & 2.06 & $\mathrm{P}$ & 25.69 \\
\hline 260.2 & 6188. & 0.368 & $0.24503 \times 10-3$ & 0.165 & 0.790 & 63.52 & 2.53 & 2.07 & $\mathrm{P}$ & 28.77 \\
\hline 232.5 & 6194. & 0.451 & $0.18699 \times 10-3$ & 0.165 & 0.790 & 63.52 & 2.08 & 2.07 & $\mathrm{P}$ & 35.91 \\
\hline 180.9 & 6198. & 0.520 & $0.11883 \times 10-3$ & 0.165 & 0.790 & 63.52 & 1.82 & 2.08 & $\mathrm{P}$ & 52.18 \\
\hline 138.8 & 6199. & 0.545 & $0.80850 \times 10-4$ & 0.165 & 0.790 & 63.52 & 1.74 & 2.09 & $\mathrm{P}$ & 53.68 \\
\hline 275.2 & 6191. & 0.597 & $0.12893 \times 10-3$ & 0.164 & 0.790 & 63.52 & 1.60 & 2.09 & $\mathrm{P}$ & 78.82 \\
\hline 282.3 & 6193. & 0.628 & $0.11176 \times 10-3$ & 0.164 & 0.790 & 63.52 & 1.52 & 2.10 & $\mathrm{P}$ & 56.80 \\
\hline 294.1 & 6195. & 0.671 & $0.82030 \times 10-4$ & 0.163 & 0.790 & 63.52 & 1.43 & 2.10 & $\mathrm{P}$ & 120.50 \\
\hline 300.7 & 6195. & 0.698 & $0.54160 \times 10-4$ & 0.163 & 0.790 & 63.52 & 1.38 & 2.11 & $\mathrm{P}$ & 136.26 \\
\hline 286.8 & 6193. & 0.715 & $0.25100 \times 10-4$ & 0.163 & 0.790 & 63.52 & 1.34 & 2.11 & $\mathrm{P}$ & 1226.14 \\
\hline 297.8 & 3785. & 0.129 & $0.47773 \times 10-3$ & 0.163 & 0.788 & 63.52 & 6.41 & 2.05 & $\mathrm{P}$ & 26.00 \\
\hline 290.9 & 3793. & 0.325 & $0.39328 \times 10-3$ & 0.163 & 0.789 & 63.52 & 2.84 & 2.06 & $\mathrm{P}$ & 34.08 \\
\hline 277.8 & 3800. & 0.474 & $0.31453 \times 10-3$ & 0.163 & 0.789 & 63.52 & 1.99 & 2.08 & $\mathrm{P}$ & 40.15 \\
\hline 255.8 & 3806. & 0.583 & $0.24057 \times 10-3$ & 0.163 & 0.789 & 63.52 & 1.64 & 2.09 & $\mathrm{P}$ & 48.45 \\
\hline 207.6 & 3812. & 0.672 & $0.15323 \times 10-3$ & 0.163 & 0.790 & 63.52 & 1.43 & 2.10 & $\mathrm{P}$ & 71.31 \\
\hline 163.6 & 3814. & 0.705 & $0.10444 \times 10-3$ & 0.163 & 0.790 & 63.52 & 1.36 & 2.11 & $\mathrm{P}$ & 73.97 \\
\hline 381.8 & 3815. & 0.774 & $0.17670 \times 10-3$ & 0.162 & 0.790 & 63.52 & 1.25 & 2.12 & $\mathrm{P}$ & 134.09 \\
\hline
\end{tabular}




\begin{tabular}{|c|c|c|c|c|c|c|c|c|c|c|}
\hline 423.7 & 3818. & 0.817 & $0.15182 \times 10-3$ & 0.162 & 0.790 & 63.52 & 1.18 & 2.13 & $\mathrm{P}$ & 85.45 \\
\hline 563.5 & 3823. & 0.876 & $0.10839 \times 10-3$ & 0.162 & 0.790 & 63.52 & 1.11 & 2.13 & $\mathrm{P}$ & 221.14 \\
\hline 1197.5 & 3825. & 0.913 & $0.67540 \times 10-4$ & 0.162 & 0.790 & 63.52 & 1.06 & 2.14 & $\mathrm{P}$ & 492.93 \\
\hline 94.8 & 8279. & 0.002 & $0.15140 \times 10-4$ & 0.162 & 0.788 & 63.52 & 33.67 & 2.04 & $\mathrm{C}$ & 755.10 \\
\hline 128.1 & 8279. & 0.012 & $0.29250 \times 10-4$ & 0.162 & 0.788 & 63.52 & 25.24 & 2.04 & $\mathrm{C}$ & 80.66 \\
\hline 145.3 & 8280. & 0.027 & $0.42320 \times 10-4$ & 0.162 & 0.788 & 63.52 & 18.38 & 2.04 & $\mathrm{C}$ & 60.15 \\
\hline 156.0 & 8281. & 0.046 & $0.54360 \times 10-4$ & 0.162 & 0.788 & 63.52 & 13.73 & 2.04 & $\mathrm{C}$ & 45.36 \\
\hline 165.0 & 8282. & 0.073 & $0.68310 \times 10-4$ & 0.162 & 0.788 & 63.52 & 10.02 & 2.04 & $\mathrm{C}$ & 33.96 \\
\hline 169.0 & 8283. & 0.090 & $0.76070 \times 10-4$ & 0.162 & 0.788 & 63.52 & 8.51 & 2.04 & $\mathrm{C}$ & 33.49 \\
\hline 254.7 & 8281. & 0.142 & $0.13505 \times 10-3$ & 0.162 & 0.788 & 63.52 & 5.91 & 2.05 & $\mathrm{C}$ & 29.80 \\
\hline 272.4 & 8284. & 0.178 & $0.15370 \times 10-3$ & 0.162 & 0.788 & 63.52 & 4.87 & 2.05 & $\mathrm{C}$ & 27.42 \\
\hline 297.8 & 8287. & 0.250 & $0.18569 \times 10-3$ & 0.162 & 0.788 & 63.52 & 3.60 & 2.06 & $\mathrm{C}$ & 26.46 \\
\hline 317.7 & 8290. & 0.331 & $0.21590 \times 10-3$ & 0.161 & 0.788 & 63.52 & 2.79 & 2.07 & $\mathrm{C}$ & 27.53 \\
\hline 335.7 & 8293. & 0.428 & $0.24754 \times 10-3$ & 0.161 & 0.788 & 63.52 & 2.20 & 2.08 & $\mathrm{C}$ & 29.87 \\
\hline 354.4 & 8297. & 0.551 & $0.28292 \times 10-3$ & 0.160 & 0.788 & 63.52 & 1.73 & 2.09 & $\mathrm{C}$ & 33.43 \\
\hline 85.4 & 11310. & 0.001 & $0.10400 \times 10-4$ & 0.162 & 0.788 & 63.52 & 35.40 & 2.04 & $\mathrm{C}$ & 802.50 \\
\hline 119.3 & 11310. & 0.008 & $0.19800 \times 10-4$ & 0.162 & 0.788 & 63.52 & 28.56 & 2.04 & $\mathrm{C}$ & 79.51 \\
\hline 137.3 & 11310. & 0.018 & $0.28500 \times 10-4$ & 0.162 & 0.788 & 63.52 & 22.22 & 2.04 & $\mathrm{C}$ & 60.65 \\
\hline 148.2 & 11310. & 0.030 & $0.36510 \times 10-4$ & 0.162 & 0.788 & 63.52 & 17.42 & 2.04 & $\mathrm{C}$ & 46.97 \\
\hline 156.6 & 11311. & 0.048 & $0.45780 \times 10-4$ & 0.162 & 0.788 & 63.52 & 13.24 & 2.04 & $\mathrm{C}$ & 34.65 \\
\hline 160.1 & 11311. & 0.060 & $0.50930 \times 10-4$ & 0.162 & 0.788 & 63.52 & 11.45 & 2.04 & $\mathrm{C}$ & 35.17 \\
\hline 242.4 & 11303. & 0.095 & $0.92390 \times 10-4$ & 0.162 & 0.787 & 63.52 & 8.16 & 2.05 & $\mathrm{C}$ & 29.47 \\
\hline 261.4 & 11304. & 0.120 & $0.10663 \times 10-3$ & 0.162 & 0.787 & 63.52 & 6.79 & 2.05 & $\mathrm{C}$ & 27.34 \\
\hline 287.3 & 11304. & 0.171 & $0.13107 \times 10-3$ & 0.161 & 0.787 & 63.52 & 5.05 & 2.05 & $\mathrm{C}$ & 25.91 \\
\hline 306.1 & 11302. & 0.228 & $0.15402 \times 10-3$ & 0.161 & 0.787 & 63.52 & 3.92 & 2.06 & $\mathrm{C}$ & 26.64 \\
\hline 320.9 & 11299. & 0.297 & $0.17795 \times 10-3$ & 0.161 & 0.787 & 63.52 & 3.09 & 2.06 & $\mathrm{C}$ & 28.35 \\
\hline 334.1 & 11295. & 0.385 & $0.20450 \times 10-3$ & 0.160 & 0.787 & 63.52 & 2.43 & 2.07 & $\mathrm{C}$ & 31.05 \\
\hline 49.6 & 5927. & 0.001 & $0.10480 \times 10-4$ & 0.163 & 0.788 & 63.52 & 34.96 & 2.04 & $\mathrm{C}$ & 1257.47 \\
\hline 98.9 & 5927. & 0.010 & $0.30440 \times 10-4$ & 0.163 & 0.788 & 63.52 & 26.50 & 2.04 & $\mathrm{C}$ & 134.87 \\
\hline 124.1 & 5928. & 0.027 & $0.48920 \times 10-4$ & 0.163 & 0.788 & 63.52 & 18.48 & 2.04 & $\mathrm{C}$ & 81.50 \\
\hline 140.2 & 5929. & 0.049 & $0.65960 \times 10-4$ & 0.163 & 0.788 & 63.52 & 13.16 & 2.04 & $\mathrm{C}$ & 76.28 \\
\hline 154.2 & 5930. & 0.082 & $0.85710 \times 10-4$ & 0.163 & 0.788 & 63.52 & 9.14 & 2.04 & $\mathrm{C}$ & 67.63 \\
\hline 160.9 & 5931. & 0.104 & $0.96710 \times 10-4$ & 0.163 & 0.788 & 63.52 & 7.60 & 2.04 & $\mathrm{C}$ & 66.25 \\
\hline 218.1 & 5931. & 0.166 & $0.15269 \times 10-3$ & 0.162 & 0.788 & 63.52 & 5.17 & 2.05 & $\mathrm{C}$ & 29.81 \\
\hline 232.7 & 5934. & 0.206 & $0.17213 \times 10-3$ & 0.162 & 0.788 & 63.52 & 4.28 & 2.05 & $\mathrm{C}$ & 28.59 \\
\hline 255.2 & 5937. & 0.287 & $0.20544 \times 10-3$ & 0.162 & 0.788 & 63.52 & 3.18 & 2.06 & $\mathrm{C}$ & 27.70 \\
\hline 274.5 & 5941. & 0.376 & $0.23701 \times 10-3$ & 0.162 & 0.788 & 63.52 & 2.48 & 2.07 & $\mathrm{C}$ & 29.30 \\
\hline 294.1 & 5946. & 0.482 & $0.27016 \times 10-3$ & 0.162 & 0.789 & 63.52 & 1.96 & 2.08 & $\mathrm{C}$ & 32.16 \\
\hline 316.9 & 5954. & 0.616 & $0.30737 \times 10-3$ & 0.162 & 0.789 & 63.52 & 1.55 & 2.10 & $\mathrm{C}$ & 36.69 \\
\hline 23.6 & 3769. & 0.000 & $0.795 \times 10-5$ & 0.164 & 0.789 & 63.52 & 35.70 & 2.03 & $\mathrm{C}$ & 2104.07 \\
\hline 70.5 & 3769. & 0.009 & $0.31760 \times 10-4$ & 0.164 & 0.789 & 63.52 & 27.12 & 2.04 & $\mathrm{C}$ & 165.28 \\
\hline 97.6 & 3769. & 0.027 & $0.53810 \times 10-4$ & 0.164 & 0.789 & 63.52 & 18.34 & 2.04 & $\mathrm{C}$ & 92.86 \\
\hline 116.0 & 3770. & 0.051 & $0.74150 \times 10-4$ & 0.164 & 0.789 & 63.52 & 12.68 & 2.04 & $\mathrm{C}$ & 95.84 \\
\hline 133.0 & 3771. & 0.089 & $0.97740 \times 10-4$ & 0.164 & 0.789 & 63.52 & 8.57 & 2.04 & $\mathrm{C}$ & 90.66 \\
\hline 141.2 & 3772. & 0.114 & $0.11089 \times 10-3$ & 0.164 & 0.789 & 63.52 & 7.03 & 2.04 & $\mathrm{C}$ & 89.63 \\
\hline 169.0 & 3773. & 0.180 & $0.15094 \times 10-3$ & 0.164 & 0.789 & 63.52 & 4.82 & 2.05 & $\mathrm{C}$ & 35.30 \\
\hline 181.1 & 3774. & 0.219 & $0.16935 \times 10-3$ & 0.164 & 0.789 & 63.52 & 4.04 & 2.05 & $\mathrm{C}$ & 33.08 \\
\hline 200.7 & 3777. & 0.299 & $0.20088 \times 10-3$ & 0.164 & 0.789 & 63.52 & 3.06 & 2.06 & $\mathrm{C}$ & 31.64 \\
\hline 218.7 & 3781. & 0.385 & $0.23077 \times 10-3$ & 0.164 & 0.790 & 63.52 & 2.42 & 2.07 & $\mathrm{C}$ & 33.14 \\
\hline
\end{tabular}




\begin{tabular}{|c|c|c|c|c|c|c|c|c|c|c|}
\hline 237.7 & 3785. & 0.488 & $0.26217 \times 10-3$ & 0.164 & 0.790 & 63.52 & 1.94 & 2.08 & $\mathrm{C}$ & 36.17 \\
\hline 261.0 & 3791. & 0.618 & $0.29743 \times 10-3$ & 0.164 & 0.790 & 63.52 & 1.55 & 2.09 & $\mathrm{C}$ & 41.12 \\
\hline 17.7 & 3773. & 0.000 & $0.753 \times 10-5$ & 0.165 & 0.789 & 63.52 & 36.09 & 2.04 & $\mathrm{C}$ & 1867.75 \\
\hline 66.4 & 3778. & 0.008 & $0.31380 \times 10-4$ & 0.165 & 0.789 & 63.52 & 28.00 & 2.03 & $\mathrm{C}$ & 166.46 \\
\hline 95.0 & 3779. & 0.025 & $0.53640 \times 10-4$ & 0.165 & 0.789 & 63.52 & 18.78 & 2.04 & $\mathrm{C}$ & 92.21 \\
\hline 114.9 & 3779. & 0.050 & $0.74180 \times 10-4$ & 0.165 & 0.789 & 63.52 & 12.89 & 2.04 & $\mathrm{C}$ & 96.32 \\
\hline 133.5 & 3781. & 0.088 & $0.98000 \times 10-4$ & 0.165 & 0.789 & 63.52 & 8.66 & 2.04 & $\mathrm{C}$ & 90.46 \\
\hline 142.5 & 3782. & 0.113 & $0.11127 \times 10-3$ & 0.165 & 0.789 & 63.52 & 7.09 & 2.04 & $\mathrm{C}$ & 89.50 \\
\hline 169.6 & 3783. & 0.178 & $0.14965 \times 10-3$ & 0.165 & 0.790 & 63.52 & 4.85 & 2.05 & $\mathrm{C}$ & 36.15 \\
\hline 182.2 & 3784. & 0.218 & $0.16808 \times 10-3$ & 0.165 & 0.790 & 63.52 & 4.07 & 2.05 & $\mathrm{C}$ & 33.59 \\
\hline 202.5 & 3787. & 0.296 & $0.19963 \times 10-3$ & 0.165 & 0.790 & 63.52 & 3.08 & 2.06 & $\mathrm{C}$ & 32.11 \\
\hline 220.6 & 3790. & 0.382 & $0.22953 \times 10-3$ & 0.165 & 0.790 & 63.52 & 2.44 & 2.07 & $\mathrm{C}$ & 33.62 \\
\hline 239.6 & 3794. & 0.485 & $0.26094 \times 10-3$ & 0.165 & 0.790 & 63.52 & 1.95 & 2.08 & $\mathrm{C}$ & 36.65 \\
\hline 262.3 & 3800. & 0.614 & $0.29619 \times 10-3$ & 0.165 & 0.791 & 63.52 & 1.56 & 2.09 & $\mathrm{C}$ & 41.55 \\
\hline 327.6 & 8231. & 0.096 & $0.35802 \times 10-3$ & 0.162 & 0.787 & 63.52 & 8.11 & 2.05 & $\mathrm{P}$ & 19.53 \\
\hline 313.6 & 8240. & 0.243 & $0.29527 \times 10-3$ & 0.161 & 0.788 & 63.52 & 3.70 & 2.06 & $\mathrm{P}$ & 22.55 \\
\hline 292.0 & 8248. & 0.354 & $0.23680 \times 10-3$ & 0.161 & 0.788 & 63.52 & 2.62 & 2.07 & $\mathrm{P}$ & 24.61 \\
\hline 261.3 & 8252. & 0.436 & $0.18215 \times 10-3$ & 0.161 & 0.788 & 63.52 & 2.16 & 2.08 & $\mathrm{P}$ & 29.94 \\
\hline 205.4 & 8255. & 0.503 & $0.11801 \times 10-3$ & 0.161 & 0.788 & 63.52 & 1.88 & 2.09 & $\mathrm{P}$ & 40.76 \\
\hline 160.6 & 8255. & 0.528 & $0.82270 \times 10-4$ & 0.161 & 0.788 & 63.52 & 1.80 & 2.09 & $\mathrm{P}$ & 42.16 \\
\hline 285.1 & 8232. & 0.579 & $0.12261 \times 10-3$ & 0.159 & 0.787 & 63.52 & 1.65 & 2.10 & $\mathrm{P}$ & 60.76 \\
\hline 291.3 & 8232. & 0.609 & $0.10742 \times 10-3$ & 0.159 & 0.787 & 63.52 & 1.57 & 2.10 & $\mathrm{P}$ & 45.75 \\
\hline 301.9 & 8231. & 0.650 & $0.81180 \times 10-4$ & 0.159 & 0.787 & 63.52 & 1.48 & 2.11 & $\mathrm{P}$ & 91.41 \\
\hline 309.8 & 8228. & 0.679 & $0.56590 \times 10-4$ & 0.158 & 0.787 & 63.52 & 1.42 & 2.11 & $\mathrm{P}$ & 104.51 \\
\hline 309.1 & 8222. & 0.698 & $0.30960 \times 10-4$ & 0.158 & 0.787 & 63.52 & 1.38 & 2.11 & $\mathrm{P}$ & 657.36 \\
\hline 150.1 & 8213. & 0.706 & $0.264 \times 10-5$ & 0.157 & 0.787 & 63.52 & 1.36 & 2.11 & $\mathrm{P}$ & 1220.37 \\
\hline 314.2 & 11122. & 0.068 & $0.25597 \times 10-3$ & 0.163 & 0.788 & 63.52 & 10.44 & 2.04 & $\mathrm{P}$ & 19.88 \\
\hline 298.1 & 11128. & 0.173 & $0.21076 \times 10-3$ & 0.163 & 0.788 & 63.52 & 4.99 & 2.05 & $\mathrm{P}$ & 23.06 \\
\hline 274.7 & 11132. & 0.252 & $0.16869 \times 10-3$ & 0.163 & 0.789 & 63.52 & 3.57 & 2.06 & $\mathrm{P}$ & 24.90 \\
\hline 242.9 & 11134. & 0.310 & $0.12955 \times 10-3$ & 0.163 & 0.789 & 63.52 & 2.96 & 2.06 & $\mathrm{P}$ & 30.64 \\
\hline 187.8 & 11132. & 0.357 & $0.83820 \times 10-4$ & 0.162 & 0.789 & 63.52 & 2.60 & 2.07 & $\mathrm{P}$ & 42.04 \\
\hline 145.5 & 11129. & 0.375 & $0.58390 \times 10-4$ & 0.162 & 0.788 & 63.52 & 2.48 & 2.07 & $\mathrm{P}$ & 43.18 \\
\hline 262.8 & 11084. & 0.413 & $0.92960 \times 10-4$ & 0.160 & 0.787 & 63.52 & 2.27 & 2.08 & $\mathrm{P}$ & 58.40 \\
\hline 262.8 & 11081. & 0.436 & $0.81720 \times 10-4$ & 0.160 & 0.787 & 63.52 & 2.16 & 2.08 & $\mathrm{P}$ & 42.59 \\
\hline 257.5 & 11074. & 0.467 & $0.62430 \times 10-4$ & 0.160 & 0.787 & 63.52 & 2.02 & 2.08 & $\mathrm{P}$ & 82.86 \\
\hline 241.5 & 11065. & 0.489 & $0.44370 \times 10-4$ & 0.159 & 0.787 & 63.52 & 1.94 & 2.08 & $\mathrm{P}$ & 87.13 \\
\hline 200.0 & 11052. & 0.504 & $0.25590 \times 10-4$ & 0.159 & 0.787 & 63.52 & 1.88 & 2.09 & $\mathrm{P}$ & 386.10 \\
\hline 67.1 & 11036. & 0.512 & $0.487 \times 10-5$ & 0.158 & 0.786 & 63.52 & 1.86 & 2.09 & $\mathrm{P}$ & 420.18 \\
\hline 313.6 & 6157. & 0.110 & $0.40882 \times 10-3$ & 0.166 & 0.790 & 63.52 & 7.26 & 2.04 & $\mathrm{P}$ & 20.84 \\
\hline 300.7 & 6166. & 0.278 & $0.33653 \times 10-3$ & 0.166 & 0.790 & 63.52 & 3.27 & 2.06 & $\mathrm{P}$ & 25.05 \\
\hline 280.6 & 6175. & 0.405 & $0.26914 \times 10-3$ & 0.166 & 0.790 & 63.52 & 2.31 & 2.07 & $\mathrm{P}$ & 27.91 \\
\hline 251.0 & 6181. & 0.497 & $0.20602 \times 10-3$ & 0.166 & 0.791 & 63.52 & 1.90 & 2.08 & $\mathrm{P}$ & 35.44 \\
\hline 195.8 & 6186. & 0.573 & $0.13176 \times 10-3$ & 0.165 & 0.791 & 63.52 & 1.66 & 2.09 & $\mathrm{P}$ & 48.55 \\
\hline 151.1 & 6188. & 0.601 & $0.90340 \times 10-4$ & 0.165 & 0.791 & 63.52 & 1.59 & 2.09 & $\mathrm{P}$ & 50.07 \\
\hline 284.7 & 6180. & 0.658 & $0.13768 \times 10-3$ & 0.164 & 0.791 & 63.52 & 1.46 & 2.10 & $\mathrm{P}$ & 75.41 \\
\hline 292.7 & 6182. & 0.691 & $0.11976 \times 10-3$ & 0.164 & 0.791 & 63.52 & 1.39 & 2.11 & $\mathrm{P}$ & 54.20 \\
\hline 307.2 & 6185. & 0.737 & $0.88680 \times 10-4$ & 0.164 & 0.791 & 63.52 & 1.31 & 2.11 & $\mathrm{P}$ & 115.50 \\
\hline 321.3 & 6186. & 0.768 & $0.59530 \times 10-4$ & 0.164 & 0.791 & 63.52 & 1.26 & 2.12 & $\mathrm{P}$ & 131.87 \\
\hline 330.6 & 6184. & 0.787 & $0.29110 \times 10-4$ & 0.163 & 0.791 & 63.52 & 1.23 & 2.12 & $\mathrm{P}$ & 2949.04 \\
\hline
\end{tabular}




\begin{tabular}{|c|c|c|c|c|c|c|c|c|c|c|}
\hline 261.2 & 3945. & 0.111 & $0.41396 \times 10-3$ & 0.165 & 0.789 & 63.52 & 7.19 & 2.04 & $\mathrm{P}$ & 25.27 \\
\hline 251.7 & 3952. & 0.281 & $0.33923 \times 10-3$ & 0.165 & 0.790 & 63.52 & 3.24 & 2.06 & $\mathrm{P}$ & 33.61 \\
\hline 236.0 & 3958. & 0.408 & $0.26957 \times 10-3$ & 0.165 & 0.790 & 63.52 & 2.29 & 2.07 & $\mathrm{P}$ & 39.23 \\
\hline 211.9 & 3963. & 0.500 & $0.20431 \times 10-3$ & 0.165 & 0.790 & 63.52 & 1.89 & 2.08 & $\mathrm{P}$ & 48.21 \\
\hline 164.3 & 3967. & 0.576 & $0.12755 \times 10-3$ & 0.165 & 0.791 & 63.52 & 1.66 & 2.09 & $\mathrm{P}$ & 71.79 \\
\hline 124.2 & 3969. & 0.602 & $0.84730 \times 10-4$ & 0.165 & 0.791 & 63.52 & 1.59 & 2.09 & $\mathrm{P}$ & 73.72 \\
\hline 285.9 & 3969. & 0.659 & $0.14899 \times 10-3$ & 0.164 & 0.791 & 63.52 & 1.45 & 2.10 & $\mathrm{P}$ & 133.00 \\
\hline 297.9 & 3971. & 0.695 & $0.12707 \times 10-3$ & 0.164 & 0.791 & 63.52 & 1.38 & 2.10 & $\mathrm{P}$ & 77.30 \\
\hline 323.4 & 3974. & 0.743 & $0.89000 \times 10-4$ & 0.164 & 0.791 & 63.52 & 1.30 & 2.11 & $\mathrm{P}$ & 199.21 \\
\hline 352.9 & 3975. & 0.772 & $0.53260 \times 10-4$ & 0.164 & 0.791 & 63.52 & 1.25 & 2.12 & $\mathrm{P}$ & 225.86 \\
\hline 414.7 & 3975. & 0.786 & $0.15970 \times 10-4$ & 0.164 & 0.791 & 63.52 & 1.23 & 2.12 & $\mathrm{P}$ & 793.96 \\
\hline 261.8 & 3857. & 0.116 & $0.43092 \times 10-3$ & 0.164 & 0.789 & 63.52 & 6.98 & 2.04 & $\mathrm{P}$ & 24.96 \\
\hline 253.5 & 3863. & 0.292 & $0.35364 \times 10-3$ & 0.164 & 0.789 & 63.52 & 3.12 & 2.06 & $\mathrm{P}$ & 34.04 \\
\hline 239.4 & 3870. & 0.426 & $0.28160 \times 10-3$ & 0.164 & 0.790 & 63.52 & 2.20 & 2.07 & $\mathrm{P}$ & 39.46 \\
\hline 216.7 & 3875. & 0.522 & $0.21407 \times 10-3$ & 0.164 & 0.790 & 63.52 & 1.82 & 2.08 & $\mathrm{P}$ & 48.72 \\
\hline 170.6 & 3879. & 0.601 & $0.13455 \times 10-3$ & 0.164 & 0.790 & 63.52 & 1.59 & 2.09 & $\mathrm{P}$ & 69.99 \\
\hline 130.7 & 3881. & 0.629 & $0.90180 \times 10-4$ & 0.164 & 0.790 & 63.52 & 1.52 & 2.10 & $\mathrm{P}$ & 72.08 \\
\hline 294.8 & 3882. & 0.688 & $0.15230 \times 10-3$ & 0.164 & 0.790 & 63.52 & 1.39 & 2.11 & $\mathrm{P}$ & 135.48 \\
\hline 313.5 & 3884. & 0.725 & $0.13069 \times 10-3$ & 0.164 & 0.790 & 63.52 & 1.33 & 2.11 & $\mathrm{P}$ & 78.08 \\
\hline 359.9 & 3887. & 0.775 & $0.93110 \times 10-4$ & 0.164 & 0.791 & 63.52 & 1.24 & 2.12 & $\mathrm{P}$ & 205.70 \\
\hline 450.9 & 3889. & 0.806 & $0.57830 \times 10-4$ & 0.163 & 0.791 & 63.52 & 1.20 & 2.12 & $\mathrm{P}$ & 253.01 \\
\hline 1890.5 & 3889. & 0.823 & $0.20990 \times 10-4$ & 0.163 & 0.791 & 63.52 & 1.17 & 2.13 & $\mathrm{P}$ & 2512.79 \\
\hline 37.9 & 3909. & 0.004 & $0.19550 \times 10-4$ & 0.164 & 0.789 & 63.52 & 31.38 & 2.04 & $\mathrm{C}$ & 188.52 \\
\hline 75.8 & 3910. & 0.017 & $0.44210 \times 10-4$ & 0.164 & 0.789 & 63.52 & 22.27 & 2.04 & $\mathrm{C}$ & 93.53 \\
\hline 104.0 & 3910. & 0.038 & $0.66950 \times 10-4$ & 0.164 & 0.789 & 63.52 & 15.18 & 2.04 & $\mathrm{C}$ & 95.86 \\
\hline 131.6 & 3911. & 0.074 & $0.93310 \times 10-4$ & 0.164 & 0.789 & 63.52 & 9.87 & 2.04 & $\mathrm{C}$ & 92.94 \\
\hline 145.3 & 3912. & 0.098 & $0.10799 \times 10-3$ & 0.164 & 0.789 & 63.52 & 7.94 & 2.04 & $\mathrm{C}$ & 92.31 \\
\hline 173.1 & 3913. & 0.161 & $0.14255 \times 10-3$ & 0.164 & 0.789 & 63.52 & 5.30 & 2.05 & $\mathrm{C}$ & 41.42 \\
\hline 185.5 & 3914. & 0.198 & $0.15817 \times 10-3$ & 0.164 & 0.789 & 63.52 & 4.43 & 2.05 & $\mathrm{C}$ & 34.18 \\
\hline 205.7 & 3917. & 0.271 & $0.18489 \times 10-3$ & 0.164 & 0.789 & 63.52 & 3.34 & 2.06 & $\mathrm{C}$ & 32.69 \\
\hline 223.4 & 3920. & 0.350 & $0.21020 \times 10-3$ & 0.164 & 0.789 & 63.52 & 2.64 & 2.07 & $\mathrm{C}$ & 33.62 \\
\hline 241.7 & 3924. & 0.444 & $0.23674 \times 10-3$ & 0.164 & 0.790 & 63.52 & 2.12 & 2.08 & $\mathrm{C}$ & 36.17 \\
\hline 262.7 & 3929. & 0.560 & $0.26643 \times 10-3$ & 0.164 & 0.790 & 63.52 & 1.70 & 2.09 & $\mathrm{C}$ & 41.02 \\
\hline 236.5 & 3757. & 0.107 & $0.39819 \times 10-3$ & 0.164 & 0.789 & 63.52 & 7.43 & 2.04 & $\mathrm{P}$ & 25.72 \\
\hline 227.5 & 3763. & 0.270 & $0.32536 \times 10-3$ & 0.164 & 0.789 & 63.52 & 3.36 & 2.06 & $\mathrm{P}$ & 36.02 \\
\hline 212.4 & 3768. & 0.392 & $0.25749 \times 10-3$ & 0.164 & 0.789 & 63.52 & 2.38 & 2.07 & $\mathrm{P}$ & 44.15 \\
\hline 189.5 & 3772. & 0.479 & $0.19396 \times 10-3$ & 0.164 & 0.790 & 63.52 & 1.97 & 2.08 & $\mathrm{P}$ & 52.05 \\
\hline 144.6 & 3776. & 0.550 & $0.11930 \times 10-3$ & 0.164 & 0.790 & 63.52 & 1.73 & 2.09 & $\mathrm{P}$ & 73.41 \\
\hline 107.0 & 3778. & 0.575 & $0.77670 \times 10-4$ & 0.164 & 0.790 & 63.52 & 1.66 & 2.09 & $\mathrm{P}$ & 75.24 \\
\hline 268.9 & 3778. & 0.630 & $0.14860 \times 10-3$ & 0.163 & 0.790 & 63.52 & 1.52 & 2.10 & $\mathrm{P}$ & 134.22 \\
\hline 281.3 & 3780. & 0.665 & $0.12711 \times 10-3$ & 0.163 & 0.790 & 63.52 & 1.44 & 2.10 & $\mathrm{P}$ & 76.53 \\
\hline 306.2 & 3783. & 0.713 & $0.89810 \times 10-4$ & 0.163 & 0.790 & 63.52 & 1.35 & 2.11 & $\mathrm{P}$ & 201.03 \\
\hline 334.7 & 3784. & 0.743 & $0.54800 \times 10-4$ & 0.163 & 0.790 & 63.52 & 1.30 & 2.11 & $\mathrm{P}$ & 225.92 \\
\hline 379.8 & 3784. & 0.758 & $0.18260 \times 10-4$ & 0.163 & 0.790 & 63.52 & 1.27 & 2.12 & $\mathrm{P}$ & 723.65 \\
\hline 49.5 & 4054. & 0.005 & $0.24030 \times 10-4$ & 0.165 & 0.789 & 63.52 & 30.24 & 2.04 & $\mathrm{C}$ & 185.09 \\
\hline 82.6 & 4055. & 0.020 & $0.46180 \times 10-4$ & 0.165 & 0.789 & 63.52 & 21.03 & 2.04 & $\mathrm{C}$ & 90.69 \\
\hline 106.8 & 4055. & 0.041 & $0.66600 \times 10-4$ & 0.165 & 0.789 & 63.52 & 14.49 & 2.04 & $\mathrm{C}$ & 94.97 \\
\hline 129.7 & 4056. & 0.076 & $0.90280 \times 10-4$ & 0.165 & 0.789 & 63.52 & 9.66 & 2.04 & $\mathrm{C}$ & 95.89 \\
\hline 141.0 & 4057. & 0.099 & $0.10347 \times 10-3$ & 0.165 & 0.789 & 63.52 & 7.86 & 2.04 & $\mathrm{C}$ & 95.10 \\
\hline
\end{tabular}




\begin{tabular}{|l|l|l|l|l|l|l|l|l|l|l|}
\hline 170.2 & 4058. & 0.160 & $0.13905 \times 10-3$ & 0.164 & 0.789 & 63.52 & 5.32 & 2.05 & $\mathrm{C}$ & 40.82 \\
\hline 182.1 & 4060. & 0.197 & $0.15484 \times 10-3$ & 0.164 & 0.789 & 63.52 & 4.46 & 2.05 & $\mathrm{C}$ & 34.14 \\
\hline 201.1 & 4062. & 0.268 & $0.18184 \times 10-3$ & 0.164 & 0.789 & 63.52 & 3.37 & 2.06 & $\mathrm{C}$ & 32.07 \\
\hline 217.8 & 4065. & 0.346 & $0.20740 \times 10-3$ & 0.164 & 0.790 & 63.52 & 2.67 & 2.07 & $\mathrm{C}$ & 33.31 \\
\hline 234.6 & 4069. & 0.438 & $0.23420 \times 10-3$ & 0.164 & 0.790 & 63.52 & 2.14 & 2.07 & $\mathrm{C}$ & 36.03 \\
\hline 254.0 & 4074. & 0.554 & $0.26419 \times 10-3$ & 0.164 & 0.790 & 63.52 & 1.72 & 2.09 & $\mathrm{C}$ & 40.94 \\
\hline
\end{tabular}




\section{APPENDIX B1}

Convective Boiling of R448A within a micro-fin tube

(file: hqgwp3.dat)

\begin{tabular}{|c|c|c|c|c|c|c|c|c|c|}
\hline $\begin{array}{c}q^{\prime \prime} \\
\left(\mathrm{Wm}^{-2}\right)\end{array}$ & $\begin{array}{l}\Delta T_{\mathrm{s}} \\
(\mathrm{K})\end{array}$ & $x_{\mathrm{q}}$ & $\begin{array}{c}G_{\mathrm{r}} \\
\left(\mathrm{kg} \mathrm{m}^{-2} \mathrm{~s}^{-1}\right)\end{array}$ & $T_{\mathrm{w}}(\mathrm{K})$ & $T_{\mathrm{s}}(\mathrm{K})$ & $T_{\mathrm{f}}(\mathrm{K})$ & $z(\mathrm{~m})$ & $\begin{array}{c}M_{\mathrm{w}} \\
(\mathrm{g} / \mathrm{mole})\end{array}$ & flow \\
\hline 3208. & 1.07 & 0.19 & 218. & 279.14 & 278.10 & 280.71 & 0.35 & 86.28 & $\mathrm{C}$ \\
\hline 3910. & 1.44 & 0.23 & 218. & 279.64 & 278.27 & 281.01 & 0.97 & 86.28 & $\mathrm{C}$ \\
\hline 4558. & 1.73 & 0.27 & 218. & 280.10 & 278.47 & 281.39 & 1.54 & 86.28 & $\mathrm{C}$ \\
\hline 5155. & 1.96 & 0.31 & 218. & 280.52 & 278.68 & 281.93 & 2.06 & 86.28 & $\mathrm{C}$ \\
\hline 5845. & 2.18 & 0.36 & 218. & 281.01 & 278.96 & 282.49 & 2.66 & 86.28 & $\mathrm{C}$ \\
\hline 6227. & 2.28 & 0.40 & 218. & 281.28 & 279.14 & 283.05 & 3.00 & 86.28 & $\mathrm{C}$ \\
\hline 9486. & 2.46 & 0.49 & 218. & 281.84 & 279.50 & 283.45 & 3.69 & 86.28 & $\mathrm{C}$ \\
\hline 9616. & 2.47 & 0.54 & 218. & 282.11 & 279.79 & 284.34 & 4.03 & 86.28 & $\mathrm{C}$ \\
\hline 9838. & 2.47 & 0.64 & 218. & 282.58 & 280.32 & 285.47 & 4.61 & 86.28 & $\mathrm{C}$ \\
\hline 10045. & 2.43 & 0.73 & 218. & 283.02 & 280.82 & 286.40 & 5.15 & 86.28 & $\mathrm{C}$ \\
\hline 10260. & 2.37 & 0.84 & 218. & 283.48 & 281.34 & 287.57 & 5.72 & 86.28 & $\mathrm{C}$ \\
\hline 10496. & 2.27 & 0.95 & 218. & 283.98 & 281.92 & 288.55 & 6.34 & 86.28 & $\mathrm{C}$ \\
\hline 3368. & 1.23 & 0.19 & 216. & 279.41 & 278.18 & 280.94 & 0.35 & 86.28 & $\mathrm{C}$ \\
\hline 4127. & 1.56 & 0.23 & 216. & 279.89 & 278.37 & 281.23 & 0.97 & 86.28 & $\mathrm{C}$ \\
\hline 4828. & 1.82 & 0.27 & 216. & 280.33 & 278.57 & 281.60 & 1.54 & 86.28 & $\mathrm{C}$ \\
\hline 5474. & 2.02 & 0.32 & 216. & 280.74 & 278.80 & 282.12 & 2.06 & 86.28 & $\mathrm{C}$ \\
\hline 6220. & 2.21 & 0.38 & 216. & 281.22 & 279.11 & 282.65 & 2.66 & 86.28 & $\mathrm{C}$ \\
\hline 6632. & 2.29 & 0.41 & 216. & 281.48 & 279.31 & 283.19 & 3.00 & 86.28 & $\mathrm{C}$ \\
\hline 9419. & 2.44 & 0.51 & 216. & 282.01 & 279.66 & 283.55 & 3.69 & 86.28 & $\mathrm{C}$ \\
\hline 9508. & 2.45 & 0.56 & 216. & 282.28 & 279.96 & 284.36 & 4.03 & 86.28 & $\mathrm{C}$ \\
\hline 9661. & 2.43 & 0.66 & 216. & 282.74 & 280.48 & 285.35 & 4.61 & 86.28 & $\mathrm{C}$ \\
\hline 9803. & 2.39 & 0.76 & 216. & 283.16 & 280.98 & 286.18 & 5.15 & 86.28 & $\mathrm{C}$ \\
\hline 9951. & 2.32 & 0.86 & 216. & 283.60 & 281.49 & 287.19 & 5.72 & 86.28 & $\mathrm{C}$ \\
\hline 10114. & 2.21 & 0.97 & 216. & 284.09 & 282.06 & 288.04 & 6.34 & 86.28 & $\mathrm{C}$ \\
\hline 2152. & 1.18 & 0.11 & 297. & 279.45 & 278.26 & 280.66 & 0.35 & 86.28 & $\mathrm{C}$ \\
\hline 2959. & 1.45 & 0.13 & 297. & 279.80 & 278.33 & 280.84 & 0.97 & 86.28 & $\mathrm{C}$ \\
\hline 3706. & 1.68 & 0.15 & 297. & 280.12 & 278.42 & 281.16 & 1.54 & 86.28 & $\mathrm{C}$ \\
\hline 4392. & 1.86 & 0.18 & 297. & 280.42 & 278.53 & 281.62 & 2.06 & 86.28 & $\mathrm{C}$ \\
\hline 5186. & 2.04 & 0.21 & 297. & 280.76 & 278.69 & 282.04 & 2.66 & 86.28 & $\mathrm{C}$ \\
\hline 5625. & 2.13 & 0.23 & 297. & 280.95 & 278.79 & 282.51 & 3.00 & 86.28 & $\mathrm{C}$ \\
\hline 9536. & 2.32 & 0.30 & 297. & 281.34 & 278.94 & 282.84 & 3.69 & 86.28 & $\mathrm{C}$ \\
\hline 9452. & 2.35 & 0.34 & 297. & 281.53 & 279.13 & 283.58 & 4.03 & 86.28 & $\mathrm{C}$ \\
\hline 9309. & 2.40 & 0.40 & 297. & 281.87 & 279.43 & 284.69 & 4.61 & 86.28 & $\mathrm{C}$ \\
\hline 9176. & 2.44 & 0.46 & 297. & 282.17 & 279.68 & 285.64 & 5.15 & 86.28 & $\mathrm{C}$ \\
\hline 9038. & 2.48 & 0.53 & 297. & 282.49 & 279.93 & 286.80 & 5.72 & 86.28 & $\mathrm{C}$ \\
\hline 8886. & 2.52 & 0.60 & 297. & 282.85 & 280.17 & 287.53 & 6.34 & 86.28 & $\mathrm{C}$ \\
\hline 2690. & 1.65 & 0.12 & 164. & 280.03 & 278.27 & 281.45 & 0.35 & 86.28 & $\mathrm{C}$ \\
\hline 3357. & 1.89 & 0.16 & 164. & 280.48 & 278.46 & 281.67 & 0.97 & 86.28 & $\mathrm{C}$ \\
\hline
\end{tabular}




\begin{tabular}{|c|c|c|c|c|c|c|c|c|c|}
\hline 3974. & 2.06 & 0.20 & 164. & 280.90 & 278.68 & 282.02 & 1.54 & 86.28 & $\mathrm{C}$ \\
\hline 4541. & 2.18 & 0.25 & 164. & 281.28 & 278.93 & 282.53 & 2.06 & 86.28 & $\mathrm{C}$ \\
\hline 5197. & 2.28 & 0.31 & 164. & 281.72 & 279.27 & 282.98 & 2.66 & 86.28 & $\mathrm{C}$ \\
\hline 5560. & 2.32 & 0.35 & 164. & 281.97 & 279.48 & 283.43 & 3.00 & 86.28 & $\mathrm{C}$ \\
\hline 7425. & 2.36 & 0.46 & 164. & 282.47 & 279.93 & 283.72 & 3.69 & 86.28 & $\mathrm{C}$ \\
\hline 7288. & 2.33 & 0.51 & 164. & 282.72 & 280.24 & 284.44 & 4.03 & 86.28 & $\mathrm{C}$ \\
\hline 7052. & 2.26 & 0.61 & 164. & 283.15 & 280.77 & 285.17 & 4.61 & 86.28 & $\mathrm{C}$ \\
\hline 6832. & 2.19 & 0.69 & 164. & 283.55 & 281.25 & 285.78 & 5.15 & 86.28 & $\mathrm{C}$ \\
\hline 6604. & 2.11 & 0.78 & 164. & 283.96 & 281.73 & 286.51 & 5.72 & 86.28 & $\mathrm{C}$ \\
\hline 6353. & 2.01 & 0.88 & 164. & 284.42 & 282.24 & 287.07 & 6.34 & 86.28 & $\mathrm{C}$ \\
\hline 2461. & 2.12 & 0.09 & 101. & 280.42 & 278.09 & 281.45 & 0.35 & 86.28 & $\mathrm{C}$ \\
\hline 2604. & 2.13 & 0.14 & 101. & 280.75 & 278.35 & 281.66 & 0.97 & 86.28 & $\mathrm{C}$ \\
\hline 2736. & 2.13 & 0.20 & 101. & 281.05 & 278.61 & 281.94 & 1.54 & 86.28 & $\mathrm{C}$ \\
\hline 2857. & 2.12 & 0.25 & 101. & 281.33 & 278.87 & 282.29 & 2.06 & 86.28 & $\mathrm{C}$ \\
\hline 2997. & 2.09 & 0.31 & 101. & 281.65 & 279.20 & 282.57 & 2.66 & 86.28 & $\mathrm{C}$ \\
\hline 3075 . & 2.06 & 0.35 & 101. & 281.83 & 279.40 & 282.82 & 3.00 & 86.28 & $\mathrm{C}$ \\
\hline 3174. & 2.03 & 0.42 & 101. & 282.20 & 279.77 & 282.92 & 3.69 & 86.28 & $\mathrm{C}$ \\
\hline 3188. & 1.99 & 0.46 & 101. & 282.38 & 279.99 & 283.33 & 4.03 & 86.28 & $\mathrm{C}$ \\
\hline 3212. & 1.91 & 0.53 & 101. & 282.69 & 280.38 & 283.61 & 4.61 & 86.28 & $\mathrm{C}$ \\
\hline 3234. & 1.84 & 0.60 & 101. & 282.98 & 280.74 & 283.91 & 5.15 & 86.28 & $\mathrm{C}$ \\
\hline 3257. & 1.76 & 0.66 & 101. & 283.28 & 281.13 & 284.24 & 5.72 & 86.28 & $\mathrm{C}$ \\
\hline 3283. & 1.66 & 0.74 & 101. & 283.62 & 281.56 & 284.53 & 6.34 & 86.28 & $\mathrm{C}$ \\
\hline 20662. & 5.06 & 0.16 & 213. & 284.03 & 278.63 & 289.36 & 0.35 & 86.28 & $\mathrm{P}$ \\
\hline 16754. & 4.06 & 0.35 & 213. & 283.87 & 279.62 & 287.64 & 0.97 & 86.28 & $\mathrm{P}$ \\
\hline 13136. & 3.24 & 0.49 & 213. & 283.72 & 280.41 & 286.47 & 1.54 & 86.28 & $\mathrm{P}$ \\
\hline 9801. & 2.59 & 0.60 & 213. & 283.59 & 280.99 & 285.56 & 2.06 & 86.28 & $\mathrm{P}$ \\
\hline 5940. & 1.98 & 0.68 & 213. & 283.43 & 281.44 & 284.91 & 2.66 & 86.28 & $\mathrm{P}$ \\
\hline 3801. & 1.72 & 0.71 & 213. & 283.34 & 281.59 & 284.49 & 3.00 & 86.28 & $\mathrm{P}$ \\
\hline 5843. & 1.41 & 0.77 & 213. & 283.16 & 281.50 & 284.15 & 3.69 & 86.28 & $\mathrm{P}$ \\
\hline 5018. & 1.17 & 0.80 & 213. & 283.07 & 281.64 & 283.88 & 4.03 & 86.28 & $\mathrm{P}$ \\
\hline 3604. & 0.84 & 0.85 & 213. & 282.92 & 281.83 & 283.42 & 4.61 & 86.28 & $\mathrm{P}$ \\
\hline 2284. & 0.60 & 0.88 & 213. & 282.77 & 281.91 & 283.26 & 5.15 & 86.28 & $\mathrm{P}$ \\
\hline 915. & 0.43 & 0.90 & 213. & 282.63 & 281.91 & 283.08 & 5.72 & 86.28 & $\mathrm{P}$ \\
\hline 19644. & 4.22 & 0.18 & 296. & 282.87 & 278.51 & 287.84 & 0.35 & 86.28 & $\mathrm{P}$ \\
\hline 16139. & 3.46 & 0.32 & 296. & 282.61 & 279.16 & 286.25 & 0.97 & 86.28 & $\mathrm{P}$ \\
\hline 12892. & 2.84 & 0.42 & 296. & 282.38 & 279.67 & 285.13 & 1.54 & 86.28 & $\mathrm{P}$ \\
\hline 9900. & 2.33 & 0.49 & 296. & 282.16 & 280.03 & 284.22 & 2.06 & 86.28 & $\mathrm{P}$ \\
\hline 6436. & 1.84 & 0.55 & 296. & 281.91 & 280.30 & 283.56 & 2.66 & 86.28 & $\mathrm{P}$ \\
\hline 4517. & 1.62 & 0.58 & 296. & 281.78 & 280.38 & 283.10 & 3.00 & 86.28 & $\mathrm{P}$ \\
\hline 7183. & 1.42 & 0.63 & 296. & 281.49 & 280.07 & 282.70 & 3.69 & 86.28 & $\mathrm{P}$ \\
\hline 6284. & 1.21 & 0.66 & 296. & 281.35 & 280.16 & 282.37 & 4.03 & 86.28 & $\mathrm{P}$ \\
\hline 4741. & 0.90 & 0.70 & 296. & 281.11 & 280.26 & 281.79 & 4.61 & 86.28 & $\mathrm{P}$ \\
\hline 3301. & 0.66 & 0.73 & 296. & 280.89 & 280.29 & 281.57 & 5.15 & 86.28 & $\mathrm{P}$ \\
\hline 1807. & 0.47 & 0.75 & 296. & 280.66 & 280.24 & 281.31 & 5.72 & 86.28 & $\mathrm{P}$ \\
\hline 162. & 0.33 & 0.76 & 296. & 280.40 & 280.10 & 281.28 & 6.34 & 86.28 & $\mathrm{P}$ \\
\hline
\end{tabular}




\begin{tabular}{|c|c|c|c|c|c|c|c|c|c|}
\hline 16982. & 4.44 & 0.21 & 162. & 283.51 & 278.76 & 287.91 & 0.35 & 86.28 & $\mathrm{P}$ \\
\hline 13648. & 3.44 & 0.42 & 162. & 283.42 & 279.88 & 286.50 & 0.97 & 86.28 & $\mathrm{P}$ \\
\hline 10561. & 2.63 & 0.57 & 162. & 283.34 & 280.78 & 285.57 & 1.54 & 86.28 & $\mathrm{P}$ \\
\hline 7716. & 2.00 & 0.68 & 162. & 283.27 & 281.43 & 284.87 & 2.06 & 86.28 & $\mathrm{P}$ \\
\hline 4423. & 1.43 & 0.77 & 162. & 283.18 & 281.92 & 284.37 & 2.66 & 86.28 & $\mathrm{P}$ \\
\hline 2599. & 1.20 & 0.80 & 162. & 283.14 & 282.07 & 284.06 & 3.00 & 86.28 & $\mathrm{P}$ \\
\hline 4294. & 0.88 & 0.85 & 162. & 283.04 & 282.13 & 283.80 & 3.69 & 86.28 & $\mathrm{P}$ \\
\hline 3634. & 0.67 & 0.88 & 162. & 282.99 & 282.30 & 283.64 & 4.03 & 86.28 & $\mathrm{P}$ \\
\hline 2501. & 0.38 & 0.93 & 162. & 282.91 & 282.50 & 283.30 & 4.61 & 86.28 & $\mathrm{P}$ \\
\hline 1444. & 0.19 & 0.95 & 162. & 282.84 & 282.60 & 283.21 & 5.15 & 86.28 & $\mathrm{P}$ \\
\hline 348. & 0.07 & 0.97 & 162. & 282.76 & 282.61 & 283.09 & 5.72 & 86.28 & $\mathrm{P}$ \\
\hline 10586. & 3.87 & 0.22 & 92. & 283.00 & 278.83 & 285.75 & 0.35 & 86.28 & $\mathrm{P}$ \\
\hline 8353. & 2.86 & 0.44 & 92. & 282.98 & 280.07 & 284.84 & 0.97 & 86.28 & $\mathrm{P}$ \\
\hline 6286. & 2.05 & 0.61 & 92. & 282.96 & 281.06 & 284.30 & 1.54 & 86.28 & $\mathrm{P}$ \\
\hline 4381. & 1.44 & 0.72 & 92. & 282.94 & 281.75 & 283.95 & 2.06 & 86.28 & $\mathrm{P}$ \\
\hline 2177. & 0.92 & 0.81 & 92. & 282.92 & 282.25 & 283.68 & 2.66 & 86.28 & $\mathrm{P}$ \\
\hline 956. & 0.73 & 0.83 & 92. & 282.91 & 282.38 & 283.51 & 3.00 & 86.28 & $\mathrm{P}$ \\
\hline 2443. & 0.41 & 0.87 & 92. & 282.89 & 282.55 & 283.35 & 3.69 & 86.28 & $\mathrm{P}$ \\
\hline 1981. & 0.22 & 0.91 & 92. & 282.88 & 282.74 & 283.31 & 4.03 & 86.28 & $\mathrm{P}$ \\
\hline 1188. & 0.00 & 0.94 & 92. & 282.86 & 282.96 & 283.09 & 4.61 & 86.28 & $\mathrm{P}$ \\
\hline 2834. & 1.85 & 0.02 & 214. & 280.02 & 278.03 & 281.42 & 0.35 & 86.28 & $\mathrm{C}$ \\
\hline 3541. & 2.11 & 0.05 & 214. & 280.47 & 278.16 & 281.65 & 0.97 & 86.28 & $\mathrm{C}$ \\
\hline 4195. & 2.31 & 0.09 & 214. & 280.88 & 278.32 & 282.00 & 1.54 & 86.28 & $\mathrm{C}$ \\
\hline 4797. & 2.47 & 0.13 & 214. & 281.26 & 278.50 & 282.53 & 2.06 & 86.28 & $\mathrm{C}$ \\
\hline 5492. & 2.63 & 0.18 & 214. & 281.71 & 278.74 & 283.00 & 2.66 & 86.28 & $\mathrm{C}$ \\
\hline 5877. & 2.69 & 0.21 & 214. & 281.95 & 278.90 & 283.47 & 3.00 & 86.28 & $\mathrm{C}$ \\
\hline 8570. & 2.84 & 0.29 & 214. & 282.45 & 279.18 & 283.85 & 3.69 & 86.28 & $\mathrm{C}$ \\
\hline 8683. & 2.85 & 0.34 & 214. & 282.70 & 279.43 & 284.59 & 4.03 & 86.28 & $\mathrm{C}$ \\
\hline 8875. & 2.85 & 0.43 & 214. & 283.13 & 279.86 & 285.50 & 4.61 & 86.28 & $\mathrm{C}$ \\
\hline 9055. & 2.83 & 0.51 & 214. & 283.52 & 280.28 & 286.26 & 5.15 & 86.28 & $\mathrm{C}$ \\
\hline 9241. & 2.79 & 0.60 & 214. & 283.94 & 280.72 & 287.16 & 5.72 & 86.28 & $\mathrm{C}$ \\
\hline 9446. & 2.72 & 0.71 & 214. & 284.39 & 281.22 & 287.99 & 6.34 & 86.28 & $\mathrm{C}$ \\
\hline 2289. & 1.15 & 0.09 & 302. & 279.38 & 278.19 & 280.69 & 0.35 & 86.28 & $\mathrm{C}$ \\
\hline 3121. & 1.45 & 0.11 & 302. & 279.76 & 278.27 & 280.87 & 0.97 & 86.28 & $\mathrm{C}$ \\
\hline 3889. & 1.70 & 0.13 & 302. & 280.12 & 278.36 & 281.18 & 1.54 & 86.28 & $\mathrm{C}$ \\
\hline 4596. & 1.91 & 0.16 & 302. & 280.44 & 278.47 & 281.65 & 2.06 & 86.28 & $\mathrm{C}$ \\
\hline 5414. & 2.12 & 0.19 & 302. & 280.82 & 278.63 & 282.09 & 2.66 & 86.28 & $\mathrm{C}$ \\
\hline 5866. & 2.23 & 0.21 & 302. & 281.03 & 278.73 & 282.54 & 3.00 & 86.28 & $\mathrm{C}$ \\
\hline 8955. & 2.48 & 0.28 & 302. & 281.47 & 278.85 & 282.86 & 3.69 & 86.28 & $\mathrm{C}$ \\
\hline 9270. & 2.54 & 0.31 & 302. & 281.68 & 279.01 & 283.63 & 4.03 & 86.28 & $\mathrm{C}$ \\
\hline 9809. & 2.63 & 0.38 & 302. & 282.04 & 279.31 & 284.62 & 4.61 & 86.28 & $\mathrm{C}$ \\
\hline 10311. & 2.71 & 0.45 & 302. & 282.39 & 279.59 & 285.47 & 5.15 & 86.28 & $\mathrm{C}$ \\
\hline 10832. & 2.76 & 0.52 & 302. & 282.74 & 279.89 & 286.53 & 5.72 & 86.28 & $\mathrm{C}$ \\
\hline 11404. & 2.81 & 0.61 & 302. & 283.13 & 280.22 & 287.48 & 6.34 & 86.28 & $\mathrm{C}$ \\
\hline 2296. & 1.19 & 0.08 & 303. & 279.38 & 278.15 & 280.70 & 0.35 & 86.28 & $\mathrm{C}$ \\
\hline
\end{tabular}




\begin{tabular}{|c|c|c|c|c|c|c|c|c|c|}
\hline 3122. & 1.49 & 0.10 & 303. & 279.77 & 278.22 & 280.87 & 0.97 & 86.28 & $\mathrm{C}$ \\
\hline 3886. & 1.73 & 0.12 & 303. & 280.12 & 278.32 & 281.19 & 1.54 & 86.28 & $\mathrm{C}$ \\
\hline 4589. & 1.94 & 0.15 & 303. & 280.45 & 278.43 & 281.66 & 2.06 & 86.28 & $\mathrm{C}$ \\
\hline 5402. & 2.15 & 0.18 & 303. & 280.83 & 278.58 & 282.09 & 2.66 & 86.28 & $\mathrm{C}$ \\
\hline 5851. & 2.26 & 0.20 & 303. & 281.04 & 278.68 & 282.54 & 3.00 & 86.28 & $\mathrm{C}$ \\
\hline 8949. & 2.51 & 0.27 & 303. & 281.46 & 278.79 & 282.86 & 3.69 & 86.28 & $\mathrm{C}$ \\
\hline 9264. & 2.57 & 0.30 & 303. & 281.68 & 278.96 & 283.63 & 4.03 & 86.28 & $\mathrm{C}$ \\
\hline 9804. & 2.66 & 0.37 & 303. & 282.04 & 279.25 & 284.62 & 4.61 & 86.28 & $\mathrm{C}$ \\
\hline 10308. & 2.73 & 0.43 & 303. & 282.38 & 279.52 & 285.47 & 5.15 & 86.28 & $\mathrm{C}$ \\
\hline 10830. & 2.79 & 0.51 & 303. & 282.73 & 279.82 & 286.53 & 5.72 & 86.28 & $\mathrm{C}$ \\
\hline 11404. & 2.84 & 0.59 & 303. & 283.12 & 280.15 & 287.47 & 6.34 & 86.28 & $\mathrm{C}$ \\
\hline 2616. & 1.82 & 0.05 & 164. & 280.07 & 278.10 & 281.42 & 0.35 & 86.28 & $\mathrm{C}$ \\
\hline 3146. & 2.03 & 0.09 & 164. & 280.51 & 278.27 & 281.66 & 0.97 & 86.28 & $\mathrm{C}$ \\
\hline 3637. & 2.20 & 0.13 & 164. & 280.92 & 278.47 & 282.01 & 1.54 & 86.28 & $\mathrm{C}$ \\
\hline 4088. & 2.33 & 0.18 & 164. & 281.30 & 278.68 & 282.52 & 2.06 & 86.28 & $\mathrm{C}$ \\
\hline 4610. & 2.44 & 0.23 & 164. & 281.74 & 278.98 & 282.95 & 2.66 & 86.28 & $\mathrm{C}$ \\
\hline 4899. & 2.48 & 0.27 & 164. & 281.98 & 279.16 & 283.38 & 3.00 & 86.28 & $\mathrm{C}$ \\
\hline 6832. & 2.55 & 0.36 & 164. & 282.47 & 279.53 & 283.73 & 3.69 & 86.28 & $\mathrm{C}$ \\
\hline 6861. & 2.54 & 0.41 & 164. & 282.72 & 279.81 & 284.40 & 4.03 & 86.28 & $\mathrm{C}$ \\
\hline 6912. & 2.50 & 0.50 & 164. & 283.14 & 280.29 & 285.16 & 4.61 & 86.28 & $\mathrm{C}$ \\
\hline 6960. & 2.44 & 0.58 & 164. & 283.53 & 280.75 & 285.81 & 5.15 & 86.28 & $\mathrm{C}$ \\
\hline 7009. & 2.36 & 0.67 & 164. & 283.94 & 281.24 & 286.59 & 5.72 & 86.28 & $\mathrm{C}$ \\
\hline 7063. & 2.26 & 0.78 & 164. & 284.39 & 281.78 & 287.28 & 6.34 & 86.28 & $\mathrm{C}$ \\
\hline 2759. & 1.94 & 0.07 & 158. & 280.26 & 278.15 & 281.57 & 0.35 & 86.28 & $\mathrm{C}$ \\
\hline 3289. & 2.11 & 0.11 & 158. & 280.68 & 278.34 & 281.79 & 0.97 & 86.28 & $\mathrm{C}$ \\
\hline 3780. & 2.24 & 0.15 & 158. & 281.07 & 278.56 & 282.13 & 1.54 & 86.28 & $\mathrm{C}$ \\
\hline 4231. & 2.34 & 0.20 & 158. & 281.42 & 278.79 & 282.61 & 2.06 & 86.28 & $\mathrm{C}$ \\
\hline 4753. & 2.41 & 0.26 & 158. & 281.84 & 279.11 & 283.01 & 2.66 & 86.28 & $\mathrm{C}$ \\
\hline 5042. & 2.43 & 0.30 & 158. & 282.07 & 279.31 & 283.41 & 3.00 & 86.28 & $\mathrm{C}$ \\
\hline 6542. & 2.48 & 0.39 & 158. & 282.54 & 279.69 & 283.71 & 3.69 & 86.28 & $\mathrm{C}$ \\
\hline 6550. & 2.45 & 0.44 & 158. & 282.77 & 279.97 & 284.31 & 4.03 & 86.28 & $\mathrm{C}$ \\
\hline 6563. & 2.39 & 0.53 & 158. & 283.17 & 280.45 & 284.93 & 4.61 & 86.28 & $\mathrm{C}$ \\
\hline 6575. & 2.33 & 0.62 & 158. & 283.54 & 280.91 & 285.48 & 5.15 & 86.28 & $\mathrm{C}$ \\
\hline 6588. & 2.24 & 0.71 & 158. & 283.93 & 281.39 & 286.12 & 5.72 & 86.28 & $\mathrm{C}$ \\
\hline 6601. & 2.13 & 0.81 & 158. & 284.35 & 281.93 & 286.70 & 6.34 & 86.28 & $\mathrm{C}$ \\
\hline 2511. & 2.11 & 0.09 & 97. & 280.64 & 278.26 & 281.71 & 0.35 & 86.28 & $\mathrm{C}$ \\
\hline 2704. & 2.11 & 0.14 & 97. & 280.99 & 278.54 & 281.90 & 0.97 & 86.28 & $\mathrm{C}$ \\
\hline 2882. & 2.10 & 0.20 & 97. & 281.32 & 278.83 & 282.21 & 1.54 & 86.28 & $\mathrm{C}$ \\
\hline 3045. & 2.07 & 0.26 & 97. & 281.62 & 279.13 & 282.61 & 2.06 & 86.28 & $\mathrm{C}$ \\
\hline 3234. & 2.02 & 0.33 & 97. & 281.96 & 279.50 & 282.91 & 2.66 & 86.28 & $\mathrm{C}$ \\
\hline 3339. & 1.99 & 0.37 & 97. & 282.15 & 279.73 & 283.17 & 3.00 & 86.28 & $\mathrm{C}$ \\
\hline 3044. & 1.95 & 0.45 & 97. & 282.55 & 280.14 & 283.27 & 3.69 & 86.28 & $\mathrm{C}$ \\
\hline 3047. & 1.91 & 0.49 & 97. & 282.74 & 280.36 & 283.63 & 4.03 & 86.28 & $\mathrm{C}$ \\
\hline 3051. & 1.86 & 0.56 & 97. & 283.07 & 280.75 & 283.90 & 4.61 & 86.28 & $\mathrm{C}$ \\
\hline 3056. & 1.80 & 0.62 & 97. & 283.39 & 281.12 & 284.19 & 5.15 & 86.28 & $\mathrm{C}$ \\
\hline
\end{tabular}




\begin{tabular}{|c|c|c|c|c|c|c|c|c|c|}
\hline 3061. & 1.75 & 0.69 & 97. & 283.71 & 281.51 & 284.47 & 5.72 & 86.28 & $\mathrm{C}$ \\
\hline 3066. & 1.68 & 0.77 & 97. & 284.06 & 281.93 & 284.74 & 6.34 & 86.28 & $\mathrm{C}$ \\
\hline 20940. & 4.96 & 0.17 & 216. & 283.93 & 278.66 & 289.20 & 0.35 & 86.28 & $\mathrm{P}$ \\
\hline 17035. & 3.97 & 0.37 & 216. & 283.77 & 279.65 & 287.53 & 0.97 & 86.28 & $\mathrm{P}$ \\
\hline 13419. & 3.15 & 0.51 & 216. & 283.61 & 280.46 & 286.38 & 1.54 & 86.28 & $\mathrm{P}$ \\
\hline 10087. & 2.50 & 0.62 & 216. & 283.47 & 281.04 & 285.45 & 2.06 & 86.28 & $\mathrm{P}$ \\
\hline 6229. & 1.90 & 0.71 & 216. & 283.31 & 281.50 & 284.81 & 2.66 & 86.28 & $\mathrm{P}$ \\
\hline 4091. & 1.64 & 0.74 & 216. & 283.22 & 281.64 & 284.38 & 3.00 & 86.28 & $\mathrm{P}$ \\
\hline 6110. & 1.31 & 0.80 & 216. & 283.03 & 281.58 & 284.04 & 3.69 & 86.28 & $\mathrm{P}$ \\
\hline 5263. & 1.08 & 0.83 & 216. & 282.94 & 281.73 & 283.77 & 4.03 & 86.28 & $\mathrm{P}$ \\
\hline 3809. & 0.74 & 0.88 & 216. & 282.78 & 281.93 & 283.30 & 4.61 & 86.28 & $\mathrm{P}$ \\
\hline 2452. & 0.50 & 0.91 & 216. & 282.64 & 282.02 & 283.14 & 5.15 & 86.28 & $\mathrm{P}$ \\
\hline 1045. & 0.33 & 0.93 & 216. & 282.48 & 282.02 & 282.96 & 5.72 & 86.28 & $\mathrm{P}$ \\
\hline 18997. & 4.32 & 0.15 & 301. & 282.86 & 278.38 & 287.68 & 0.35 & 86.28 & $\mathrm{P}$ \\
\hline 15678. & 3.58 & 0.28 & 301. & 282.60 & 278.99 & 286.15 & 0.97 & 86.28 & $\mathrm{P}$ \\
\hline 12604. & 2.97 & 0.37 & 301. & 282.36 & 279.46 & 285.08 & 1.54 & 86.28 & $\mathrm{P}$ \\
\hline 9771. & 2.46 & 0.45 & 301. & 282.14 & 279.79 & 284.17 & 2.06 & 86.28 & $\mathrm{P}$ \\
\hline 6492. & 1.98 & 0.51 & 301. & 281.88 & 280.05 & 283.52 & 2.66 & 86.28 & $\mathrm{P}$ \\
\hline 4675. & 1.75 & 0.53 & 301. & 281.74 & 280.13 & 283.06 & 3.00 & 86.28 & $\mathrm{P}$ \\
\hline 7347. & 1.54 & 0.58 & 301. & 281.45 & 279.82 & 282.65 & 3.69 & 86.28 & $\mathrm{P}$ \\
\hline 6429. & 1.33 & 0.61 & 301. & 281.30 & 279.91 & 282.33 & 4.03 & 86.28 & $\mathrm{P}$ \\
\hline 4854. & 1.01 & 0.65 & 301. & 281.06 & 280.01 & 281.73 & 4.61 & 86.28 & $\mathrm{P}$ \\
\hline 3383. & 0.77 & 0.68 & 301. & 280.83 & 280.04 & 281.51 & 5.15 & 86.28 & $\mathrm{P}$ \\
\hline 1858. & 0.57 & 0.70 & 301. & 280.59 & 279.99 & 281.25 & 5.72 & 86.28 & $\mathrm{P}$ \\
\hline 178. & 0.43 & 0.71 & 301. & 280.33 & 279.85 & 281.22 & 6.34 & 86.28 & $\mathrm{P}$ \\
\hline 15912. & 4.43 & 0.15 & 169. & 283.34 & 278.57 & 287.50 & 0.35 & 86.28 & $\mathrm{P}$ \\
\hline 12814. & 3.52 & 0.34 & 169. & 283.24 & 279.53 & 286.17 & 0.97 & 86.28 & $\mathrm{P}$ \\
\hline 9946. & 2.77 & 0.48 & 169. & 283.16 & 280.31 & 285.29 & 1.54 & 86.28 & $\mathrm{P}$ \\
\hline 7303. & 2.19 & 0.58 & 169. & 283.08 & 280.87 & 284.62 & 2.06 & 86.28 & $\mathrm{P}$ \\
\hline 4243. & 1.66 & 0.66 & 169. & 282.99 & 281.30 & 284.16 & 2.66 & 86.28 & $\mathrm{P}$ \\
\hline 2548. & 1.43 & 0.68 & 169. & 282.94 & 281.43 & 283.85 & 3.00 & 86.28 & $\mathrm{P}$ \\
\hline 4272. & 1.13 & 0.73 & 169. & 282.83 & 281.44 & 283.60 & 3.69 & 86.28 & $\mathrm{P}$ \\
\hline 3617. & 0.93 & 0.76 & 169. & 282.78 & 281.60 & 283.44 & 4.03 & 86.28 & $\mathrm{P}$ \\
\hline 2494. & 0.65 & 0.80 & 169. & 282.70 & 281.80 & 283.08 & 4.61 & 86.28 & $\mathrm{P}$ \\
\hline 1445. & 0.46 & 0.83 & 169. & 282.61 & 281.89 & 282.99 & 5.15 & 86.28 & $\mathrm{P}$ \\
\hline 357. & 0.35 & 0.84 & 169. & 282.53 & 281.89 & 282.87 & 5.72 & 86.28 & $\mathrm{P}$ \\
\hline 10003. & 3.81 & 0.20 & 91. & 282.82 & 278.71 & 285.42 & 0.35 & 86.28 & $\mathrm{P}$ \\
\hline 7901. & 2.84 & 0.42 & 91. & 282.80 & 279.89 & 284.57 & 0.97 & 86.28 & $\mathrm{P}$ \\
\hline 5955. & 2.06 & 0.58 & 91. & 282.79 & 280.83 & 284.05 & 1.54 & 86.28 & $\mathrm{P}$ \\
\hline 4162. & 1.47 & 0.69 & 91. & 282.77 & 281.49 & 283.73 & 2.06 & 86.28 & $\mathrm{P}$ \\
\hline 2087. & 0.96 & 0.77 & 91. & 282.75 & 281.98 & 283.48 & 2.66 & 86.28 & $\mathrm{P}$ \\
\hline 938. & 0.77 & 0.79 & 91. & 282.74 & 282.11 & 283.32 & 3.00 & 86.28 & $\mathrm{P}$ \\
\hline 2345. & 0.46 & 0.83 & 91. & 282.72 & 282.27 & 283.17 & 3.69 & 86.28 & $\mathrm{P}$ \\
\hline 1893. & 0.28 & 0.86 & 91. & 282.71 & 282.46 & 283.14 & 4.03 & 86.28 & $\mathrm{P}$ \\
\hline 1118. & 0.05 & 0.90 & 91. & 282.70 & 282.67 & 282.93 & 4.61 & 86.28 & $\mathrm{P}$ \\
\hline
\end{tabular}




\begin{tabular}{|c|c|c|c|c|c|c|c|c|c|}
\hline 395. & -.08 & 0.92 & 91. & 282.68 & 282.75 & 282.91 & 5.15 & 86.28 & $\mathrm{P}$ \\
\hline
\end{tabular}




\section{APPENDIX B2}

Convective Boiling of R449A within a micro-fin tube

(file: hfqgwp3.dat)

\begin{tabular}{|c|c|c|c|c|c|c|c|c|c|}
\hline $\begin{array}{c}q^{\prime \prime} \\
\left(\mathrm{Wm}^{-2}\right)\end{array}$ & $\begin{array}{l}\Delta T_{\mathrm{s}} \\
(\mathrm{K})\end{array}$ & $x_{\mathrm{q}}$ & $\begin{array}{c}G_{\mathrm{r}} \\
\left(\mathrm{kg} \mathrm{m}^{-2} \mathrm{~s}^{-1}\right) \\
\end{array}$ & $T_{\mathrm{w}}(\mathrm{K})$ & $T_{\mathrm{s}}(\mathrm{K})$ & $T_{\mathrm{f}}(\mathrm{K})$ & $z(\mathrm{~m})$ & $\begin{array}{c}M_{\mathrm{w}} \\
(\mathrm{g} / \mathrm{mole})\end{array}$ & flow \\
\hline 1077. & 1.34 & 0.08 & 231. & 279.50 & 278.14 & 280.96 & 0.35 & 87.21 & $\mathrm{C}$ \\
\hline 2185. & 1.67 & 0.10 & 231. & 279.91 & 278.21 & 281.02 & 0.97 & 87.21 & $\mathrm{C}$ \\
\hline 3209. & 1.93 & 0.12 & 231. & 280.29 & 278.33 & 281.33 & 1.54 & 87.21 & $\mathrm{C}$ \\
\hline 4152. & 2.13 & 0.15 & 231. & 280.64 & 278.48 & 281.85 & 2.06 & 87.21 & $\mathrm{C}$ \\
\hline 5241. & 2.31 & 0.20 & 231. & 281.04 & 278.71 & 282.33 & 2.66 & 87.21 & $\mathrm{C}$ \\
\hline 5844. & 2.39 & 0.23 & 231. & 281.26 & 278.86 & 282.82 & 3.00 & 87.21 & $\mathrm{C}$ \\
\hline 8808. & 2.54 & 0.31 & 231. & 281.72 & 279.15 & 283.21 & 3.69 & 87.21 & $\mathrm{C}$ \\
\hline 9171. & 2.55 & 0.36 & 231. & 281.95 & 279.39 & 284.04 & 4.03 & 87.21 & $\mathrm{C}$ \\
\hline 9792. & 2.56 & 0.45 & 231. & 282.34 & 279.81 & 285.20 & 4.61 & 87.21 & $\mathrm{C}$ \\
\hline 10370. & 2.53 & 0.54 & 231. & 282.70 & 280.21 & 286.15 & 5.15 & 87.21 & $\mathrm{C}$ \\
\hline 10970. & 2.48 & 0.63 & 231. & 283.08 & 280.65 & 287.36 & 5.72 & 87.21 & $\mathrm{C}$ \\
\hline 11630. & 2.39 & 0.75 & 231. & 283.49 & 281.15 & 288.47 & 6.34 & 87.21 & $\mathrm{C}$ \\
\hline 2283. & 0.90 & 0.08 & 298. & 279.03 & 278.13 & 280.33 & 0.35 & 87.21 & $\mathrm{C}$ \\
\hline 3031. & 1.22 & 0.11 & 298. & 279.42 & 278.22 & 280.53 & 0.97 & 87.21 & $\mathrm{C}$ \\
\hline 3722. & 1.49 & 0.13 & 298. & 279.79 & 278.32 & 280.85 & 1.54 & 87.21 & $\mathrm{C}$ \\
\hline 4358. & 1.72 & 0.15 & 298. & 280.12 & 278.44 & 281.35 & 2.06 & 87.21 & $\mathrm{C}$ \\
\hline 5094. & 1.96 & 0.19 & 298. & 280.51 & 278.60 & 281.83 & 2.66 & 87.21 & $\mathrm{C}$ \\
\hline 5500. & 2.07 & 0.21 & 298. & 280.73 & 278.70 & 282.32 & 3.00 & 87.21 & $\mathrm{C}$ \\
\hline 8757. & 2.34 & 0.27 & 298. & 281.17 & 278.84 & 282.70 & 3.69 & 87.21 & $\mathrm{C}$ \\
\hline 9168. & 2.41 & 0.31 & 298. & 281.39 & 279.02 & 283.51 & 4.03 & 87.21 & $\mathrm{C}$ \\
\hline 9872. & 2.52 & 0.38 & 298. & 281.76 & 279.31 & 284.66 & 4.61 & 87.21 & $\mathrm{C}$ \\
\hline 10528. & 2.62 & 0.45 & 298. & 282.11 & 279.58 & 285.64 & 5.15 & 87.21 & $\mathrm{C}$ \\
\hline 11208. & 2.70 & 0.53 & 298. & 282.47 & 279.86 & 286.89 & 5.72 & 87.21 & $\mathrm{C}$ \\
\hline 11955. & 2.78 & 0.62 & 298. & 282.87 & 280.17 & 288.02 & 6.34 & 87.21 & $\mathrm{C}$ \\
\hline 2425. & 1.67 & 0.02 & 162. & 280.01 & 278.27 & 281.34 & 0.35 & 87.21 & $\mathrm{C}$ \\
\hline 2934. & 1.88 & 0.05 & 162. & 280.46 & 278.45 & 281.56 & 0.97 & 87.21 & $\mathrm{C}$ \\
\hline 3404. & 2.05 & 0.09 & 162. & 280.87 & 278.66 & 281.89 & 1.54 & 87.21 & $\mathrm{C}$ \\
\hline 3837. & 2.18 & 0.14 & 162. & 281.24 & 278.88 & 282.40 & 2.06 & 87.21 & $\mathrm{C}$ \\
\hline 4338. & 2.29 & 0.19 & 162. & 281.68 & 279.17 & 282.86 & 2.66 & 87.21 & $\mathrm{C}$ \\
\hline 4615. & 2.34 & 0.23 & 162. & 281.92 & 279.36 & 283.27 & 3.00 & 87.21 & $\mathrm{C}$ \\
\hline 6516. & 2.43 & 0.31 & 162. & 282.42 & 279.73 & 283.57 & 3.69 & 87.21 & $\mathrm{C}$ \\
\hline 6591. & 2.43 & 0.37 & 162. & 282.67 & 279.99 & 284.24 & 4.03 & 87.21 & $\mathrm{C}$ \\
\hline 6718. & 2.43 & 0.45 & 162. & 283.09 & 280.43 & 285.05 & 4.61 & 87.21 & $\mathrm{C}$ \\
\hline 6838. & 2.41 & 0.54 & 162. & 283.48 & 280.84 & 285.73 & 5.15 & 87.21 & $\mathrm{C}$ \\
\hline 6961. & 2.39 & 0.63 & 162. & 283.89 & 281.27 & 286.54 & 5.72 & 87.21 & $\mathrm{C}$ \\
\hline 7097. & 2.34 & 0.73 & 162. & 284.34 & 281.74 & 287.25 & 6.34 & 87.21 & $\mathrm{C}$ \\
\hline 965. & 1.63 & 0.01 & 104. & 279.77 & 278.02 & 280.97 & 0.35 & 87.21 & $\mathrm{C}$ \\
\hline
\end{tabular}




\begin{tabular}{|c|c|c|c|c|c|c|c|c|c|}
\hline 1482. & 1.78 & 0.04 & 104. & 280.14 & 278.15 & 281.06 & 0.97 & 87.21 & $\mathrm{C}$ \\
\hline 1960. & 1.88 & 0.07 & 104. & 280.48 & 278.33 & 281.34 & 1.54 & 87.21 & $\mathrm{C}$ \\
\hline 2400. & 1.94 & 0.11 & 104. & 280.79 & 278.53 & 281.78 & 2.06 & 87.21 & $\mathrm{C}$ \\
\hline 2908. & 1.99 & 0.17 & 104. & 281.16 & 278.83 & 282.15 & 2.66 & 87.21 & $\mathrm{C}$ \\
\hline 3190. & 1.99 & 0.20 & 104. & 281.36 & 279.02 & 282.51 & 3.00 & 87.21 & $\mathrm{C}$ \\
\hline 3989. & 1.97 & 0.29 & 104. & 281.78 & 279.46 & 282.73 & 3.69 & 87.21 & $\mathrm{C}$ \\
\hline 4004. & 1.93 & 0.34 & 104. & 281.98 & 279.72 & 283.29 & 4.03 & 87.21 & $\mathrm{C}$ \\
\hline 4030. & 1.86 & 0.42 & 104. & 282.33 & 280.17 & 283.90 & 4.61 & 87.21 & $\mathrm{C}$ \\
\hline 4054. & 1.79 & 0.50 & 104. & 282.66 & 280.58 & 284.44 & 5.15 & 87.21 & $\mathrm{C}$ \\
\hline 4079. & 1.72 & 0.58 & 104. & 283.00 & 281.01 & 285.10 & 5.72 & 87.21 & $\mathrm{C}$ \\
\hline 4107. & 1.63 & 0.68 & 104. & 283.38 & 281.48 & 285.62 & 6.34 & 87.21 & $\mathrm{C}$ \\
\hline 24022. & 5.28 & 0.14 & 223. & 284.17 & 278.73 & 292.39 & 0.35 & 87.21 & $\mathrm{P}$ \\
\hline 19258. & 4.12 & 0.36 & 223. & 283.87 & 279.85 & 289.33 & 0.97 & 87.21 & $\mathrm{P}$ \\
\hline 14844. & 3.19 & 0.52 & 223. & 283.60 & 280.67 & 287.46 & 1.54 & 87.21 & $\mathrm{P}$ \\
\hline 10774. & 2.47 & 0.64 & 223. & 283.34 & 281.22 & 285.95 & 2.06 & 87.21 & $\mathrm{P}$ \\
\hline 6061. & 1.79 & 0.72 & 223. & 283.05 & 281.62 & 284.93 & 2.66 & 87.21 & $\mathrm{P}$ \\
\hline 3449. & 1.49 & 0.75 & 223. & 282.89 & 281.72 & 284.26 & 3.00 & 87.21 & $\mathrm{P}$ \\
\hline 5579. & 1.12 & 0.80 & 223. & 282.55 & 281.54 & 283.77 & 3.69 & 87.21 & $\mathrm{P}$ \\
\hline 4818. & 0.85 & 0.83 & 223. & 282.39 & 281.64 & 283.35 & 4.03 & 87.21 & $\mathrm{P}$ \\
\hline 3513. & 0.45 & 0.87 & 223. & 282.11 & 281.77 & 282.71 & 4.61 & 87.21 & $\mathrm{P}$ \\
\hline 2294. & 0.14 & 0.90 & 223. & 281.84 & 281.81 & 282.47 & 5.15 & 87.21 & $\mathrm{P}$ \\
\hline 1030. & -.11 & 0.91 & 223. & 281.57 & 281.78 & 282.21 & 5.72 & 87.21 & $\mathrm{P}$ \\
\hline 24095. & 5.28 & 0.14 & 224. & 284.18 & 278.76 & 292.36 & 0.35 & 87.21 & $\mathrm{P}$ \\
\hline 19321. & 4.12 & 0.36 & 224. & 283.88 & 279.87 & 289.32 & 0.97 & 87.21 & $\mathrm{P}$ \\
\hline 14897. & 3.20 & 0.52 & 224. & 283.60 & 280.69 & 287.45 & 1.54 & 87.21 & $\mathrm{P}$ \\
\hline 10818. & 2.47 & 0.63 & 224. & 283.35 & 281.25 & 285.94 & 2.06 & 87.21 & $\mathrm{P}$ \\
\hline 6095. & 1.79 & 0.72 & 224. & 283.06 & 281.65 & 284.94 & 2.66 & 87.21 & $\mathrm{P}$ \\
\hline 3477. & 1.49 & 0.75 & 224. & 282.89 & 281.75 & 284.27 & 3.00 & 87.21 & $\mathrm{P}$ \\
\hline 5598. & 1.11 & 0.79 & 224. & 282.56 & 281.56 & 283.78 & 3.69 & 87.21 & $\mathrm{P}$ \\
\hline 4843. & 0.84 & 0.82 & 224. & 282.39 & 281.67 & 283.35 & 4.03 & 87.21 & $\mathrm{P}$ \\
\hline 3549. & 0.44 & 0.87 & 224. & 282.11 & 281.79 & 282.71 & 4.61 & 87.21 & $\mathrm{P}$ \\
\hline 2341. & 0.13 & 0.89 & 224. & 281.84 & 281.83 & 282.47 & 5.15 & 87.21 & $\mathrm{P}$ \\
\hline 1087. & 0.00 & 0.91 & 224. & 281.57 & 281.81 & 282.21 & 5.72 & 87.21 & $\mathrm{P}$ \\
\hline 22606. & 4.97 & 0.10 & 296. & 283.64 & 278.57 & 291.41 & 0.35 & 87.21 & $\mathrm{P}$ \\
\hline 18269. & 4.00 & 0.26 & 296. & 283.26 & 279.33 & 288.49 & 0.97 & 87.21 & $\mathrm{P}$ \\
\hline 14250. & 3.22 & 0.37 & 296. & 282.91 & 279.88 & 286.72 & 1.54 & 87.21 & $\mathrm{P}$ \\
\hline 10544. & 2.60 & 0.45 & 296. & 282.58 & 280.25 & 285.27 & 2.06 & 87.21 & $\mathrm{P}$ \\
\hline 6253. & 1.99 & 0.52 & 296. & 282.20 & 280.50 & 284.23 & 2.66 & 87.21 & $\mathrm{P}$ \\
\hline 3874. & 1.70 & 0.54 & 296. & 281.99 & 280.56 & 283.51 & 3.00 & 87.21 & $\mathrm{P}$ \\
\hline 6286. & 1.40 & 0.58 & 296. & 281.56 & 280.18 & 282.94 & 3.69 & 87.21 & $\mathrm{P}$ \\
\hline 5489. & 1.14 & 0.61 & 296. & 281.35 & 280.23 & 282.47 & 4.03 & 87.21 & $\mathrm{P}$ \\
\hline 4120. & 0.75 & 0.64 & 296. & 280.98 & 280.28 & 281.71 & 4.61 & 87.21 & $\mathrm{P}$ \\
\hline 2841. & 0.43 & 0.67 & 296. & 280.64 & 280.26 & 281.42 & 5.15 & 87.21 & $\mathrm{P}$ \\
\hline 1515. & 0.17 & 0.68 & 296. & 280.29 & 280.18 & 281.09 & 5.72 & 87.21 & $\mathrm{P}$ \\
\hline 54. & 0.00 & 0.69 & 296. & 279.90 & 280.02 & 281.06 & 6.34 & 87.21 & $\mathrm{P}$ \\
\hline
\end{tabular}




\begin{tabular}{|c|c|c|c|c|c|c|c|c|c|}
\hline 22804. & 4.98 & 0.10 & 299. & 283.69 & 278.63 & 291.40 & 0.35 & 87.21 & $\mathrm{P}$ \\
\hline 18421. & 4.01 & 0.26 & 299. & 283.30 & 279.39 & 288.48 & 0.97 & 87.21 & $\mathrm{P}$ \\
\hline 14361. & 3.22 & 0.37 & 299. & 282.94 & 279.95 & 286.72 & 1.54 & 87.21 & $\mathrm{P}$ \\
\hline 10617. & 2.59 & 0.45 & 299. & 282.62 & 280.31 & 285.27 & 2.06 & 87.21 & $\mathrm{P}$ \\
\hline 6282. & 1.97 & 0.52 & 299. & 282.24 & 280.56 & 284.23 & 2.66 & 87.21 & $\mathrm{P}$ \\
\hline 3879. & 1.69 & 0.54 & 299. & 282.03 & 280.62 & 283.52 & 3.00 & 87.21 & $\mathrm{P}$ \\
\hline 6325. & 1.38 & 0.58 & 299. & 281.59 & 280.24 & 282.96 & 3.69 & 87.21 & $\mathrm{P}$ \\
\hline 5521. & 1.12 & 0.61 & 299. & 281.38 & 280.30 & 282.48 & 4.03 & 87.21 & $\mathrm{P}$ \\
\hline 4143. & 0.72 & 0.64 & 299. & 281.01 & 280.35 & 281.73 & 4.61 & 87.21 & $\mathrm{P}$ \\
\hline 2856. & 0.41 & 0.67 & 299. & 280.67 & 280.33 & 281.44 & 5.15 & 87.21 & $\mathrm{P}$ \\
\hline 1521. & 0.14 & 0.68 & 299. & 280.32 & 280.25 & 281.11 & 5.72 & 87.21 & $\mathrm{P}$ \\
\hline 50. & -.09 & 0.69 & 299. & 279.93 & 280.09 & 281.08 & 6.34 & 87.21 & $\mathrm{P}$ \\
\hline 18936. & 4.66 & 0.16 & 157. & 283.72 & 278.89 & 290.45 & 0.35 & 87.21 & $\mathrm{P}$ \\
\hline 15100. & 3.50 & 0.40 & 157. & 283.54 & 280.18 & 288.00 & 0.97 & 87.21 & $\mathrm{P}$ \\
\hline 11546. & 2.58 & 0.58 & 157. & 283.38 & 281.13 & 286.52 & 1.54 & 87.21 & $\mathrm{P}$ \\
\hline 8269. & 1.88 & 0.71 & 157. & 283.23 & 281.78 & 285.30 & 2.06 & 87.21 & $\mathrm{P}$ \\
\hline 4475. & 1.24 & 0.80 & 157. & 283.05 & 282.25 & 284.51 & 2.66 & 87.21 & $\mathrm{P}$ \\
\hline 2372. & 0.98 & 0.83 & 157. & 282.96 & 282.37 & 284.01 & 3.00 & 87.21 & $\mathrm{P}$ \\
\hline 3913. & 0.61 & 0.88 & 157. & 282.76 & 282.36 & 283.65 & 3.69 & 87.21 & $\mathrm{P}$ \\
\hline 3332. & 0.38 & 0.91 & 157. & 282.66 & 282.50 & 283.38 & 4.03 & 87.21 & $\mathrm{P}$ \\
\hline 2334. & 0.04 & 0.95 & 157. & 282.49 & 282.67 & 282.93 & 4.61 & 87.21 & $\mathrm{P}$ \\
\hline 1402. & -.19 & 0.97 & 157. & 282.33 & 282.74 & 282.78 & 5.15 & 87.21 & $\mathrm{P}$ \\
\hline 436. & -.36 & 0.98 & 157. & 282.17 & 282.74 & 282.62 & 5.72 & 87.21 & $\mathrm{P}$ \\
\hline 11520. & 4.07 & 0.16 & 96. & 283.46 & 279.16 & 287.69 & 0.35 & 87.21 & $\mathrm{P}$ \\
\hline 9046. & 2.97 & 0.40 & 96. & 283.39 & 280.45 & 286.10 & 0.97 & 87.21 & $\mathrm{P}$ \\
\hline 6754. & 2.13 & 0.58 & 96. & 283.33 & 281.39 & 285.22 & 1.54 & 87.21 & $\mathrm{P}$ \\
\hline 4642. & 1.50 & 0.69 & 96. & 283.27 & 282.03 & 284.51 & 2.06 & 87.21 & $\mathrm{P}$ \\
\hline 2197. & 0.96 & 0.78 & 96. & 283.20 & 282.47 & 284.07 & 2.66 & 87.21 & $\mathrm{P}$ \\
\hline 843. & 0.75 & 0.80 & 96. & 283.16 & 282.58 & 283.81 & 3.00 & 87.21 & $\mathrm{P}$ \\
\hline 2068. & 0.43 & 0.83 & 96. & 283.09 & 282.67 & 283.60 & 3.69 & 87.21 & $\mathrm{P}$ \\
\hline 1696. & 0.24 & 0.85 & 96. & 283.05 & 282.81 & 283.50 & 4.03 & 87.21 & $\mathrm{P}$ \\
\hline 1058. & -.00 & 0.89 & 96. & 282.98 & 282.97 & 283.24 & 4.61 & 87.21 & $\mathrm{P}$ \\
\hline 462. & -.15 & 0.90 & 96. & 282.92 & 283.03 & 283.19 & 5.15 & 87.21 & $\mathrm{P}$ \\
\hline 1264. & 1.23 & 0.00 & 220. & 279.54 & 278.35 & 281.03 & 0.35 & 87.21 & $\mathrm{C}$ \\
\hline 2137. & 1.55 & 0.02 & 220. & 279.99 & 278.43 & 281.16 & 0.97 & 87.21 & $\mathrm{C}$ \\
\hline 2944. & 1.80 & 0.05 & 220. & 280.41 & 278.54 & 281.48 & 1.54 & 87.21 & $\mathrm{C}$ \\
\hline 3687. & 2.01 & 0.07 & 220. & 280.80 & 278.69 & 282.02 & 2.06 & 87.21 & $\mathrm{C}$ \\
\hline 4545. & 2.20 & 0.12 & 220. & 281.25 & 278.90 & 282.55 & 2.66 & 87.21 & $\mathrm{C}$ \\
\hline 5019. & 2.29 & 0.14 & 220. & 281.50 & 279.04 & 283.11 & 3.00 & 87.21 & $\mathrm{C}$ \\
\hline 9263. & 2.43 & 0.22 & 220. & 282.01 & 279.37 & 283.65 & 3.69 & 87.21 & $\mathrm{C}$ \\
\hline 9707. & 2.44 & 0.28 & 220. & 282.26 & 279.64 & 284.70 & 4.03 & 87.21 & $\mathrm{C}$ \\
\hline 10468. & 2.43 & 0.38 & 220. & 282.70 & 280.12 & 286.31 & 4.61 & 87.21 & $\mathrm{C}$ \\
\hline 11177. & 2.39 & 0.48 & 220. & 283.10 & 280.59 & 287.62 & 5.15 & 87.21 & $\mathrm{C}$ \\
\hline 11912. & 2.33 & 0.59 & 220. & 283.52 & 281.10 & 289.32 & 5.72 & 87.21 & $\mathrm{C}$ \\
\hline 12720. & 2.22 & 0.72 & 220. & 283.98 & 281.68 & 290.87 & 6.34 & 87.21 & $\mathrm{C}$ \\
\hline
\end{tabular}




\begin{tabular}{|c|c|c|c|c|c|c|c|c|c|}
\hline 2115. & 1.17 & 0.01 & 221. & 279.70 & 278.55 & 281.15 & 0.35 & 87.21 & $\mathrm{C}$ \\
\hline 2796. & 1.50 & 0.03 & 221. & 280.18 & 278.67 & 281.40 & 0.97 & 87.21 & $\mathrm{C}$ \\
\hline 3426. & 1.76 & 0.06 & 221. & 280.62 & 278.81 & 281.74 & 1.54 & 87.21 & $\mathrm{C}$ \\
\hline 4005. & 1.98 & 0.10 & 221. & 281.03 & 278.97 & 282.30 & 2.06 & 87.21 & $\mathrm{C}$ \\
\hline 4674. & 2.20 & 0.14 & 221. & 281.50 & 279.20 & 282.83 & 2.66 & 87.21 & $\mathrm{C}$ \\
\hline 5044. & 2.30 & 0.17 & 221. & 281.77 & 279.34 & 283.41 & 3.00 & 87.21 & $\mathrm{C}$ \\
\hline 9220. & 2.47 & 0.25 & 221. & 282.30 & 279.66 & 283.94 & 3.69 & 87.21 & $\mathrm{C}$ \\
\hline 9700. & 2.50 & 0.30 & 221. & 282.57 & 279.93 & 285.01 & 4.03 & 87.21 & $\mathrm{C}$ \\
\hline 10524. & 2.51 & 0.40 & 221. & 283.03 & 280.41 & 286.54 & 4.61 & 87.21 & $\mathrm{C}$ \\
\hline 11291. & 2.50 & 0.50 & 221. & 283.45 & 280.88 & 287.82 & 5.15 & 87.21 & $\mathrm{C}$ \\
\hline 12086. & 2.45 & 0.61 & 221. & 283.89 & 281.39 & 289.49 & 5.72 & 87.21 & $\mathrm{C}$ \\
\hline 12960. & 2.37 & 0.75 & 221. & 284.38 & 281.99 & 291.06 & 6.34 & 87.21 & $\mathrm{C}$ \\
\hline 1636. & 0.86 & 0.01 & 304. & 279.23 & 278.37 & 280.57 & 0.35 & 87.21 & $\mathrm{C}$ \\
\hline 2335. & 1.19 & 0.02 & 304. & 279.64 & 278.44 & 280.76 & 0.97 & 87.21 & $\mathrm{C}$ \\
\hline 2982. & 1.46 & 0.04 & 304. & 280.02 & 278.52 & 281.04 & 1.54 & 87.21 & $\mathrm{C}$ \\
\hline 3577. & 1.70 & 0.06 & 304. & 280.37 & 278.62 & 281.54 & 2.06 & 87.21 & $\mathrm{C}$ \\
\hline 4264. & 1.94 & 0.09 & 304. & 280.78 & 278.75 & 282.02 & 2.66 & 87.21 & $\mathrm{C}$ \\
\hline 4645. & 2.07 & 0.11 & 304. & 281.01 & 278.84 & 282.56 & 3.00 & 87.21 & $\mathrm{C}$ \\
\hline 9179. & 2.33 & 0.16 & 304. & 281.47 & 278.99 & 283.05 & 3.69 & 87.21 & $\mathrm{C}$ \\
\hline 9820. & 2.39 & 0.20 & 304. & 281.70 & 279.18 & 284.10 & 4.03 & 87.21 & $\mathrm{C}$ \\
\hline 10918. & 2.49 & 0.28 & 304. & 282.09 & 279.51 & 285.75 & 4.61 & 87.21 & $\mathrm{C}$ \\
\hline 11942. & 2.57 & 0.35 & 304. & 282.46 & 279.83 & 287.12 & 5.15 & 87.21 & $\mathrm{C}$ \\
\hline 13001. & 2.63 & 0.44 & 304. & 282.84 & 280.17 & 288.93 & 5.72 & 87.21 & $\mathrm{C}$ \\
\hline 14166. & 2.68 & 0.54 & 304. & 283.26 & 280.56 & 290.64 & 6.34 & 87.21 & $\mathrm{C}$ \\
\hline 1549. & 0.96 & 0.01 & 298. & 279.37 & 278.42 & 280.61 & 0.35 & 87.21 & $\mathrm{C}$ \\
\hline 2269. & 1.26 & 0.02 & 298. & 279.76 & 278.48 & 280.79 & 0.97 & 87.21 & $\mathrm{C}$ \\
\hline 2934. & 1.51 & 0.04 & 298. & 280.12 & 278.56 & 281.08 & 1.54 & 87.21 & $\mathrm{C}$ \\
\hline 3546. & 1.72 & 0.06 & 298. & 280.45 & 278.66 & 281.57 & 2.06 & 87.21 & $\mathrm{C}$ \\
\hline 4253. & 1.93 & 0.09 & 298. & 280.83 & 278.80 & 282.06 & 2.66 & 87.21 & $\mathrm{C}$ \\
\hline 4644. & 2.04 & 0.11 & 298. & 281.04 & 278.89 & 282.60 & 3.00 & 87.21 & $\mathrm{C}$ \\
\hline 8995. & 2.26 & 0.16 & 298. & 281.48 & 279.05 & 283.11 & 3.69 & 87.21 & $\mathrm{C}$ \\
\hline 9728. & 2.31 & 0.20 & 298. & 281.70 & 279.24 & 284.15 & 4.03 & 87.21 & $\mathrm{C}$ \\
\hline 10984. & 2.37 & 0.28 & 298. & 282.07 & 279.58 & 285.82 & 4.61 & 87.21 & $\mathrm{C}$ \\
\hline 12154. & 2.41 & 0.36 & 298. & 282.41 & 279.91 & 287.20 & 5.15 & 87.21 & $\mathrm{C}$ \\
\hline 13365. & 2.43 & 0.45 & 298. & 282.77 & 280.27 & 289.06 & 5.72 & 87.21 & $\mathrm{C}$ \\
\hline 14697. & 2.42 & 0.56 & 298. & 283.16 & 280.70 & 290.87 & 6.34 & 87.21 & $\mathrm{C}$ \\
\hline 1798. & 1.26 & 0.01 & 168. & 279.45 & 278.15 & 280.74 & 0.35 & 87.21 & $\mathrm{C}$ \\
\hline 2316. & 1.51 & 0.04 & 168. & 279.87 & 278.29 & 280.96 & 0.97 & 87.21 & $\mathrm{C}$ \\
\hline 2793. & 1.71 & 0.07 & 168. & 280.27 & 278.44 & 281.27 & 1.54 & 87.21 & $\mathrm{C}$ \\
\hline 3233. & 1.87 & 0.11 & 168. & 280.63 & 278.62 & 281.77 & 2.06 & 87.21 & $\mathrm{C}$ \\
\hline 3741. & 2.02 & 0.15 & 168. & 281.04 & 278.85 & 282.24 & 2.66 & 87.21 & $\mathrm{C}$ \\
\hline 4022. & 2.08 & 0.18 & 168. & 281.27 & 279.00 & 282.72 & 3.00 & 87.21 & $\mathrm{C}$ \\
\hline 6941. & 2.16 & 0.26 & 168. & 281.75 & 279.36 & 283.14 & 3.69 & 87.21 & $\mathrm{C}$ \\
\hline 7152. & 2.15 & 0.32 & 168. & 281.98 & 279.63 & 284.01 & 4.03 & 87.21 & $\mathrm{C}$ \\
\hline 7513. & 2.10 & 0.41 & 168. & 282.38 & 280.11 & 285.19 & 4.61 & 87.21 & $\mathrm{C}$ \\
\hline
\end{tabular}




\begin{tabular}{|c|c|c|c|c|c|c|c|c|c|}
\hline 7849. & 2.03 & 0.50 & 168. & 282.76 & 280.57 & 286.19 & 5.15 & 87.21 & $\mathrm{C}$ \\
\hline 8198. & 1.94 & 0.60 & 168. & 283.15 & 281.06 & 287.43 & 5.72 & 87.21 & $\mathrm{C}$ \\
\hline 8581. & 1.81 & 0.72 & 168. & 283.58 & 281.61 & 288.52 & 6.34 & 87.21 & $\mathrm{C}$ \\
\hline 603. & 1.65 & 0.01 & 102. & 279.97 & 278.16 & 281.20 & 0.35 & 87.21 & $\mathrm{C}$ \\
\hline 1230. & 1.83 & 0.03 & 102. & 280.33 & 278.26 & 281.24 & 0.97 & 87.21 & $\mathrm{C}$ \\
\hline 1810. & 1.96 & 0.06 & 102. & 280.67 & 278.42 & 281.48 & 1.54 & 87.21 & $\mathrm{C}$ \\
\hline 2344. & 2.04 & 0.10 & 102. & 280.99 & 278.63 & 281.91 & 2.06 & 87.21 & $\mathrm{C}$ \\
\hline 2961. & 2.08 & 0.15 & 102. & 281.35 & 278.93 & 282.31 & 2.66 & 87.21 & $\mathrm{C}$ \\
\hline 3302. & 2.08 & 0.19 & 102. & 281.55 & 279.13 & 282.68 & 3.00 & 87.21 & $\mathrm{C}$ \\
\hline 4224. & 2.03 & 0.29 & 102. & 281.96 & 279.60 & 282.90 & 3.69 & 87.21 & $\mathrm{C}$ \\
\hline 4199. & 1.98 & 0.34 & 102. & 282.16 & 279.89 & 283.50 & 4.03 & 87.21 & $\mathrm{C}$ \\
\hline 4156. & 1.88 & 0.43 & 102. & 282.51 & 280.36 & 284.16 & 4.61 & 87.21 & $\mathrm{C}$ \\
\hline 4116. & 1.77 & 0.51 & 102. & 282.83 & 280.79 & 284.73 & 5.15 & 87.21 & $\mathrm{C}$ \\
\hline 4075. & 1.66 & 0.59 & 102. & 283.17 & 281.23 & 285.38 & 5.72 & 87.21 & $\mathrm{C}$ \\
\hline 4029. & 1.53 & 0.69 & 102. & 283.54 & 281.70 & 285.90 & 6.34 & 87.21 & $\mathrm{C}$ \\
\hline 581. & 1.68 & 0.01 & 100. & 280.00 & 278.17 & 281.21 & 0.35 & 87.21 & $\mathrm{C}$ \\
\hline 1199. & 1.85 & 0.03 & 100. & 280.36 & 278.28 & 281.24 & 0.97 & 87.21 & $\mathrm{C}$ \\
\hline 1770. & 1.97 & 0.06 & 100. & 280.70 & 278.44 & 281.48 & 1.54 & 87.21 & $\mathrm{C}$ \\
\hline 2296. & 2.04 & 0.10 & 100. & 281.01 & 278.64 & 281.92 & 2.06 & 87.21 & $\mathrm{C}$ \\
\hline 2903. & 2.07 & 0.15 & 100. & 281.37 & 278.94 & 282.31 & 2.66 & 87.21 & $\mathrm{C}$ \\
\hline 3239. & 2.07 & 0.19 & 100. & 281.57 & 279.15 & 282.67 & 3.00 & 87.21 & $\mathrm{C}$ \\
\hline 4164. & 2.02 & 0.29 & 100. & 281.97 & 279.62 & 282.90 & 3.69 & 87.21 & $\mathrm{C}$ \\
\hline 4145. & 1.96 & 0.34 & 100. & 282.17 & 279.90 & 283.50 & 4.03 & 87.21 & $\mathrm{C}$ \\
\hline 4113. & 1.86 & 0.43 & 100. & 282.52 & 280.38 & 284.16 & 4.61 & 87.21 & $\mathrm{C}$ \\
\hline 4083. & 1.76 & 0.51 & 100. & 282.84 & 280.83 & 284.73 & 5.15 & 87.21 & $\mathrm{C}$ \\
\hline 4051. & 1.65 & 0.60 & 100. & 283.18 & 281.27 & 285.39 & 5.72 & 87.21 & $\mathrm{C}$ \\
\hline 4017. & 1.51 & 0.69 & 100. & 283.55 & 281.76 & 285.91 & 6.34 & 87.21 & $\mathrm{C}$ \\
\hline 24165. & 5.19 & 0.15 & 216. & 284.28 & 278.89 & 292.76 & 0.35 & 87.21 & $\mathrm{P}$ \\
\hline 19362. & 4.02 & 0.38 & 216. & 284.00 & 280.05 & 289.70 & 0.97 & 87.21 & $\mathrm{P}$ \\
\hline 14912. & 3.09 & 0.54 & 216. & 283.74 & 280.91 & 287.76 & 1.54 & 87.21 & $\mathrm{P}$ \\
\hline 10809. & 2.36 & 0.66 & 216. & 283.51 & 281.49 & 286.15 & 2.06 & 87.21 & $\mathrm{P}$ \\
\hline 6057. & 1.69 & 0.75 & 216. & 283.23 & 281.91 & 285.10 & 2.66 & 87.21 & $\mathrm{P}$ \\
\hline 3423. & 1.40 & 0.78 & 216. & 283.08 & 282.01 & 284.46 & 3.00 & 87.21 & $\mathrm{P}$ \\
\hline 5492. & 1.02 & 0.83 & 216. & 282.77 & 281.86 & 283.95 & 3.69 & 87.21 & $\mathrm{P}$ \\
\hline 4736. & 0.76 & 0.86 & 216. & 282.62 & 281.97 & 283.55 & 4.03 & 87.21 & $\mathrm{P}$ \\
\hline 3440. & 0.37 & 0.90 & 216. & 282.35 & 282.10 & 282.94 & 4.61 & 87.21 & $\mathrm{P}$ \\
\hline 2230. & 0.08 & 0.93 & 216. & 282.11 & 282.15 & 282.70 & 5.15 & 87.21 & $\mathrm{P}$ \\
\hline 974. & -.16 & 0.94 & 216. & 281.85 & 282.13 & 282.46 & 5.72 & 87.21 & $\mathrm{P}$ \\
\hline 22510. & 4.80 & 0.10 & 299. & 283.66 & 278.79 & 291.56 & 0.35 & 87.21 & $\mathrm{P}$ \\
\hline 18178. & 3.85 & 0.25 & 299. & 283.29 & 279.54 & 288.71 & 0.97 & 87.21 & $\mathrm{P}$ \\
\hline 14164. & 3.08 & 0.37 & 299. & 282.95 & 280.09 & 286.88 & 1.54 & 87.21 & $\mathrm{P}$ \\
\hline 10463. & 2.47 & 0.45 & 299. & 282.64 & 280.44 & 285.29 & 2.06 & 87.21 & $\mathrm{P}$ \\
\hline 6176. & 1.87 & 0.51 & 299. & 282.28 & 280.69 & 284.23 & 2.66 & 87.21 & $\mathrm{P}$ \\
\hline 3801. & 1.60 & 0.53 & 299. & 282.08 & 280.74 & 283.57 & 3.00 & 87.21 & $\mathrm{P}$ \\
\hline 6174. & 1.31 & 0.57 & 299. & 281.67 & 280.37 & 283.01 & 3.69 & 87.21 & $\mathrm{P}$ \\
\hline
\end{tabular}




\begin{tabular}{|c|c|c|c|c|c|c|c|c|c|}
\hline 5380. & 1.06 & 0.60 & 299. & 281.47 & 280.42 & 282.54 & 4.03 & 87.21 & $\mathrm{P}$ \\
\hline 4018. & 0.69 & 0.63 & 299. & 281.12 & 280.46 & 281.82 & 4.61 & 87.21 & $\mathrm{P}$ \\
\hline 2745 & 0.39 & 0.66 & 299. & 280.80 & 280.44 & 281.53 & 5.15 & 87.21 & $\mathrm{P}$ \\
\hline 1425. & 0.15 & 0.67 & 299. & 280.46 & 280.36 & 281.21 & 5.72 & 87.21 & $\mathrm{P}$ \\
\hline 22237. & 4.82 & 0.10 & 299. & 283.58 & 278.66 & 291.46 & 0.35 & 87.21 & $\mathrm{P}$ \\
\hline 17926. & 3.88 & 0.25 & 299. & 283.21 & 279.40 & 288.55 & 0.97 & 87.21 & $\mathrm{P}$ \\
\hline 13931. & 3.12 & 0.36 & 299. & 282.87 & 279.94 & 286.76 & 1.54 & 87.21 & $\mathrm{P}$ \\
\hline 10248. & 2.51 & 0.44 & 299. & 282.55 & 280.28 & 285.18 & 2.06 & 87.21 & $\mathrm{P}$ \\
\hline 5983. & 1.92 & 0.50 & 299. & 282.19 & 280.52 & 284.12 & 2.66 & 87.21 & $\mathrm{P}$ \\
\hline 3619. & 1.65 & 0.52 & 299. & 281.99 & 280.56 & 283.46 & 3.00 & 87.21 & $\mathrm{P}$ \\
\hline 5991. & 1.36 & 0.56 & 299. & 281.57 & 280.18 & 282.91 & 3.69 & 87.21 & $\mathrm{P}$ \\
\hline 5219. & 1.12 & 0.59 & 299. & 281.37 & 280.23 & 282.44 & 4.03 & 87.21 & $\mathrm{P}$ \\
\hline 3894. & 0.74 & 0.62 & 299. & 281.02 & 280.26 & 281.72 & 4.61 & 87.21 & $\mathrm{P}$ \\
\hline 2657. & 0.45 & 0.64 & 299. & 280.69 & 280.24 & 281.43 & 5.15 & 87.21 & $\mathrm{P}$ \\
\hline 1373. & 0.20 & 0.66 & 299. & 280.35 & 280.15 & 281.11 & 5.72 & 87.21 & $\mathrm{P}$ \\
\hline 18816. & 4.71 & 0.15 & 169. & 283.57 & 278.68 & 290.33 & 0.35 & 87.21 & $\mathrm{P}$ \\
\hline 14939. & 3.60 & 0.37 & 169. & 283.38 & 279.85 & 287.82 & 0.97 & 87.21 & $\mathrm{P}$ \\
\hline 11346. & 2.73 & 0.53 & 169. & 283.22 & 280.71 & 286.33 & 1.54 & 87.21 & $\mathrm{P}$ \\
\hline 8034. & 2.06 & 0.65 & 169. & 283.06 & 281.29 & 285.16 & 2.06 & 87.21 & $\mathrm{P}$ \\
\hline 4199. & 1.46 & 0.73 & 169. & 282.88 & 281.70 & 284.39 & 2.66 & 87.21 & $\mathrm{P}$ \\
\hline 2074. & 1.21 & 0.75 & 169. & 282.78 & 281.80 & 283.91 & 3.00 & 87.21 & $\mathrm{P}$ \\
\hline 4033. & 0.85 & 0.79 & 169. & 282.58 & 281.75 & 283.52 & 3.69 & 87.21 & $\mathrm{P}$ \\
\hline 3438. & 0.63 & 0.82 & 169. & 282.48 & 281.88 & 283.24 & 4.03 & 87.21 & $\mathrm{P}$ \\
\hline 2418. & 0.29 & 0.86 & 169. & 282.30 & 282.04 & 282.76 & 4.61 & 87.21 & $\mathrm{P}$ \\
\hline 1465. & 0.06 & 0.88 & 169. & 282.14 & 282.10 & 282.60 & 5.15 & 87.21 & $\mathrm{P}$ \\
\hline 477. & -.12 & 0.90 & 169. & 281.97 & 282.10 & 282.43 & 5.72 & 87.21 & $\mathrm{P}$ \\
\hline 11090. & 3.89 & 0.14 & 105. & 282.90 & 278.81 & 287.19 & 0.35 & 87.21 & $\mathrm{P}$ \\
\hline 8801. & 2.90 & 0.35 & 105. & 282.81 & 279.94 & 285.68 & 0.97 & 87.21 & $\mathrm{P}$ \\
\hline 6680. & 2.13 & 0.51 & 105. & 282.73 & 280.77 & 284.77 & 1.54 & 87.21 & $\mathrm{P}$ \\
\hline 4726. & 1.55 & 0.61 & 105. & 282.66 & 281.34 & 284.05 & 2.06 & 87.21 & $\mathrm{P}$ \\
\hline 2464. & 1.04 & 0.69 & 105. & 282.58 & 281.76 & 283.56 & 2.66 & 87.21 & $\mathrm{P}$ \\
\hline 1210. & 0.82 & 0.71 & 105. & 282.53 & 281.87 & 283.28 & 3.00 & 87.21 & $\mathrm{P}$ \\
\hline 2429. & 0.49 & 0.75 & 105. & 282.44 & 281.98 & 283.03 & 3.69 & 87.21 & $\mathrm{P}$ \\
\hline 2009. & 0.30 & 0.78 & 105. & 282.39 & 282.12 & 282.92 & 4.03 & 87.21 & $\mathrm{P}$ \\
\hline 1288. & 0.04 & 0.81 & 105. & 282.31 & 282.30 & 282.61 & 4.61 & 87.21 & $\mathrm{P}$ \\
\hline 615. & -.12 & 0.83 & 105. & 282.24 & 282.37 & 282.54 & 5.15 & 87.21 & $\mathrm{P}$ \\
\hline 11420. & 3.98 & 0.14 & 106. & 283.15 & 278.95 & 287.54 & 0.35 & 87.21 & $\mathrm{P}$ \\
\hline 9052. & 2.97 & 0.36 & 106. & 283.07 & 280.10 & 285.99 & 0.97 & 87.21 & $\mathrm{P}$ \\
\hline 6858. & 2.19 & 0.52 & 106. & 282.99 & 280.94 & 285.05 & 1.54 & 87.21 & $\mathrm{P}$ \\
\hline 4836. & 1.59 & 0.62 & 106. & 282.92 & 281.52 & 284.30 & 2.06 & 87.21 & $\mathrm{P}$ \\
\hline 2495. & 1.07 & 0.70 & 106. & 282.83 & 281.94 & 283.81 & 2.66 & 87.21 & $\mathrm{P}$ \\
\hline 1199. & 0.86 & 0.72 & 106. & 282.78 & 282.06 & 283.53 & 3.00 & 87.21 & $\mathrm{P}$ \\
\hline 2446. & 0.52 & 0.76 & 106. & 282.69 & 282.16 & 283.27 & 3.69 & 87.21 & $\mathrm{P}$ \\
\hline 2023. & 0.33 & 0.79 & 106. & 282.64 & 282.31 & 283.15 & 4.03 & 87.21 & $\mathrm{P}$ \\
\hline 1296. & 0.07 & 0.82 & 106. & 282.56 & 282.48 & 282.85 & 4.61 & 87.21 & $\mathrm{P}$ \\
\hline
\end{tabular}




\begin{tabular}{|c|c|c|c|c|c|c|c|c|c|}
\hline 618. & -.09 & 0.84 & 106. & 282.48 & 282.55 & 282.78 & 5.15 & 87.21 & $\mathrm{P}$ \\
\hline 18270. & 4.79 & 0.14 & 169. & 283.78 & 278.80 & 290.52 & 0.35 & 87.21 & $\mathrm{P}$ \\
\hline 14634. & 3.69 & 0.36 & 169. & 283.59 & 279.94 & 288.12 & 0.97 & 87.21 & $\mathrm{P}$ \\
\hline 11265. & 2.81 & 0.52 & 169. & 283.40 & 280.78 & 286.61 & 1.54 & 87.21 & $\mathrm{P}$ \\
\hline 8159. & 2.14 & 0.63 & 169. & 283.23 & 281.36 & 285.36 & 2.06 & 87.21 & $\mathrm{P}$ \\
\hline 4562. & 1.52 & 0.72 & 169. & 283.04 & 281.78 & 284.54 & 2.66 & 87.21 & $\mathrm{P}$ \\
\hline 2569. & 1.25 & 0.75 & 169. & 282.93 & 281.90 & 284.04 & 3.00 & 87.21 & $\mathrm{P}$ \\
\hline 4082. & 0.87 & 0.79 & 169. & 282.71 & 281.88 & 283.64 & 3.69 & 87.21 & $\mathrm{P}$ \\
\hline 3485. & 0.63 & 0.82 & 169. & 282.60 & 282.00 & 283.35 & 4.03 & 87.21 & $\mathrm{P}$ \\
\hline 2460. & 0.29 & 0.86 & 169. & 282.41 & 282.16 & 282.88 & 4.61 & 87.21 & $\mathrm{P}$ \\
\hline 1504. & 0.04 & 0.88 & 169. & 282.24 & 282.23 & 282.72 & 5.15 & 87.21 & $\mathrm{P}$ \\
\hline 511. & -.15 & 0.90 & 169. & 282.05 & 282.23 & 282.55 & 5.72 & 87.21 & $\mathrm{P}$ \\
\hline 2093. & 1.41 & 0.01 & 213. & 279.59 & 278.22 & 280.98 & 0.35 & 87.21 & $\mathrm{C}$ \\
\hline 2678. & 1.71 & 0.03 & 213. & 280.04 & 278.34 & 281.25 & 0.97 & 87.21 & $\mathrm{C}$ \\
\hline 3218. & 1.95 & 0.06 & 213. & 280.47 & 278.49 & 281.59 & 1.54 & 87.21 & $\mathrm{C}$ \\
\hline 3715. & 2.14 & 0.09 & 213. & 280.86 & 278.64 & 282.14 & 2.06 & 87.21 & $\mathrm{C}$ \\
\hline 4289. & 2.33 & 0.14 & 213. & 281.31 & 278.86 & 282.62 & 2.66 & 87.21 & $\mathrm{C}$ \\
\hline 4607. & 2.42 & 0.16 & 213. & 281.56 & 278.99 & 283.18 & 3.00 & 87.21 & $\mathrm{C}$ \\
\hline 8967. & 2.56 & 0.24 & 213. & 282.08 & 279.31 & 283.75 & 3.69 & 87.21 & $\mathrm{C}$ \\
\hline 9393. & 2.56 & 0.30 & 213. & 282.33 & 279.58 & 284.80 & 4.03 & 87.21 & $\mathrm{C}$ \\
\hline 10123. & 2.54 & 0.40 & 213. & 282.77 & 280.06 & 286.35 & 4.61 & 87.21 & $\mathrm{C}$ \\
\hline 10804. & 2.50 & 0.49 & 213. & 283.17 & 280.53 & 287.67 & 5.15 & 87.21 & $\mathrm{C}$ \\
\hline 11509. & 2.42 & 0.60 & 213. & 283.60 & 281.04 & 289.36 & 5.72 & 87.21 & $\mathrm{C}$ \\
\hline 12284. & 2.31 & 0.74 & 213. & 284.06 & 281.62 & 290.92 & 6.34 & 87.21 & $\mathrm{C}$ \\
\hline 2009. & 1.53 & 0.01 & 217. & 279.73 & 278.36 & 281.10 & 0.35 & 87.21 & $\mathrm{C}$ \\
\hline 2581. & 1.79 & 0.03 & 217. & 280.18 & 278.48 & 281.36 & 0.97 & 87.21 & $\mathrm{C}$ \\
\hline 3110. & 2.00 & 0.06 & 217. & 280.60 & 278.61 & 281.70 & 1.54 & 87.21 & $\mathrm{C}$ \\
\hline 3596. & 2.17 & 0.09 & 217. & 280.98 & 278.76 & 282.24 & 2.06 & 87.21 & $\mathrm{C}$ \\
\hline 4159. & 2.34 & 0.13 & 217. & 281.43 & 278.96 & 282.72 & 2.66 & 87.21 & $\mathrm{C}$ \\
\hline 4470. & 2.42 & 0.15 & 217. & 281.67 & 279.09 & 283.27 & 3.00 & 87.21 & $\mathrm{C}$ \\
\hline 8723. & 2.55 & 0.23 & 217. & 282.18 & 279.39 & 283.83 & 3.69 & 87.21 & $\mathrm{C}$ \\
\hline 9140. & 2.55 & 0.28 & 217. & 282.43 & 279.65 & 284.87 & 4.03 & 87.21 & $\mathrm{C}$ \\
\hline 9855. & 2.54 & 0.37 & 217. & 282.86 & 280.11 & 286.41 & 4.61 & 87.21 & $\mathrm{C}$ \\
\hline 10521. & 2.51 & 0.47 & 217. & 283.26 & 280.55 & 287.71 & 5.15 & 87.21 & $\mathrm{C}$ \\
\hline 11211. & 2.45 & 0.58 & 217. & 283.67 & 281.03 & 289.39 & 5.72 & 87.21 & $\mathrm{C}$ \\
\hline 11969. & 2.36 & 0.70 & 217. & 284.13 & 281.59 & 290.94 & 6.34 & 87.21 & $\mathrm{C}$ \\
\hline 2136. & 1.27 & 0.01 & 217. & 279.36 & 278.13 & 280.82 & 0.35 & 87.21 & $\mathrm{C}$ \\
\hline 2803. & 1.58 & 0.03 & 217. & 279.84 & 278.26 & 281.09 & 0.97 & 87.21 & $\mathrm{C}$ \\
\hline 3420. & 1.84 & 0.06 & 217. & 280.28 & 278.41 & 281.44 & 1.54 & 87.21 & $\mathrm{C}$ \\
\hline 3987. & 2.04 & 0.10 & 217. & 280.69 & 278.57 & 282.00 & 2.06 & 87.21 & $\mathrm{C}$ \\
\hline 4642. & 2.25 & 0.14 & 217. & 281.17 & 278.80 & 282.54 & 2.66 & 87.21 & $\mathrm{C}$ \\
\hline 5005. & 2.35 & 0.17 & 217. & 281.43 & 278.94 & 283.14 & 3.00 & 87.21 & $\mathrm{C}$ \\
\hline 8717. & 2.52 & 0.25 & 217. & 281.97 & 279.25 & 283.68 & 3.69 & 87.21 & $\mathrm{C}$ \\
\hline 9171. & 2.54 & 0.30 & 217. & 282.23 & 279.51 & 284.69 & 4.03 & 87.21 & $\mathrm{C}$ \\
\hline 9949. & 2.56 & 0.39 & 217. & 282.69 & 279.97 & 286.21 & 4.61 & 87.21 & $\mathrm{C}$ \\
\hline
\end{tabular}




\begin{tabular}{|c|l|l|l|l|l|l|l|l|l|}
\hline 10674. & 2.55 & 0.49 & 217. & 283.11 & 280.42 & 287.48 & 5.15 & 87.21 & $\mathrm{C}$ \\
\hline 11424. & 2.52 & 0.60 & 217. & 283.55 & 280.91 & 289.12 & 5.72 & 87.21 & $\mathrm{C}$ \\
\hline 12250. & 2.46 & 0.73 & 217. & 284.04 & 281.47 & 290.65 & 6.34 & 87.21 & $\mathrm{C}$ \\
\hline 2139. & 1.39 & 0.01 & 219. & 279.42 & 278.20 & 280.89 & 0.35 & 87.21 & $\mathrm{C}$ \\
\hline 2820. & 1.67 & 0.03 & 219. & 279.90 & 278.33 & 281.15 & 0.97 & 87.21 & $\mathrm{C}$ \\
\hline 3450. & 1.90 & 0.06 & 219. & 280.35 & 278.48 & 281.50 & 1.54 & 87.21 & $\mathrm{C}$ \\
\hline 4030. & 2.09 & 0.09 & 219. & 280.76 & 278.64 & 282.06 & 2.06 & 87.21 & $\mathrm{C}$ \\
\hline 4699. & 2.27 & 0.14 & 219. & 281.24 & 278.87 & 282.61 & 2.66 & 87.21 & $\mathrm{C}$ \\
\hline 5069. & 2.36 & 0.17 & 219. & 281.50 & 279.01 & 283.21 & 3.00 & 87.21 & $\mathrm{C}$ \\
\hline 8871. & 2.52 & 0.25 & 219. & 282.04 & 279.33 & 283.75 & 3.69 & 87.21 & $\mathrm{C}$ \\
\hline 9348. & 2.54 & 0.30 & 219. & 282.31 & 279.59 & 284.76 & 4.03 & 87.21 & $\mathrm{C}$ \\
\hline 10165. & 2.55 & 0.40 & 219. & 282.77 & 280.05 & 286.30 & 4.61 & 87.21 & $\mathrm{C}$ \\
\hline 10926. & 2.54 & 0.49 & 219. & 283.19 & 280.51 & 287.58 & 5.15 & 87.21 & $\mathrm{C}$ \\
\hline 11715. & 2.51 & 0.60 & 219. & 283.64 & 281.00 & 289.24 & 5.72 & 87.21 & $\mathrm{C}$ \\
\hline 12582. & 2.45 & 0.73 & 219. & 284.13 & 281.58 & 290.80 & 6.34 & 87.21 & $\mathrm{C}$ \\
\hline
\end{tabular}




\section{APPENDIX B3}

Convective Boiling of R452B within a micro-fin tube

(file: hqgwp3.dat)

\begin{tabular}{|c|c|c|c|c|c|c|c|c|c|}
\hline $\begin{array}{c}q^{\prime \prime} \\
\left(\mathrm{Wm}^{-2}\right)\end{array}$ & $\begin{array}{l}\Delta T_{\mathrm{s}} \\
(\mathrm{K})\end{array}$ & $x_{\mathrm{q}}$ & $\begin{array}{c}G_{\mathrm{r}} \\
\left(\mathrm{kg} \mathrm{m}^{-2} \mathrm{~s}^{-1}\right)\end{array}$ & $T_{\mathrm{w}}(\mathrm{K})$ & $T_{\mathrm{s}}(\mathrm{K})$ & $T_{\mathrm{f}}(\mathrm{K})$ & $z(\mathrm{~m})$ & $\begin{array}{c}M_{\mathrm{w}} \\
(\mathrm{g} / \mathrm{mole})\end{array}$ & flow \\
\hline 1028. & 0.26 & 0.01 & 208. & 277.72 & 277.34 & 278.70 & 0.35 & 63.52 & $\mathrm{C}$ \\
\hline 1754. & 0.50 & 0.02 & 208. & 277.95 & 277.34 & 278.77 & 0.97 & 63.52 & $\mathrm{C}$ \\
\hline 2426. & 0.71 & 0.03 & 208. & 278.16 & 277.35 & 278.98 & 1.54 & 63.52 & $\mathrm{C}$ \\
\hline 3044. & 0.90 & 0.05 & 208. & 278.35 & 277.36 & 279.35 & 2.06 & 63.52 & $\mathrm{C}$ \\
\hline 3758. & 1.11 & 0.08 & 208. & 278.57 & 277.38 & 279.71 & 2.66 & 63.52 & $\mathrm{C}$ \\
\hline 4154. & 1.23 & 0.10 & 208. & 278.70 & 277.39 & 280.06 & 3.00 & 63.52 & $\mathrm{C}$ \\
\hline 7716. & 1.48 & 0.15 & 208. & 278.95 & 277.36 & 280.36 & 3.69 & 63.52 & $\mathrm{C}$ \\
\hline 8649. & 1.59 & 0.19 & 208. & 279.08 & 277.39 & 281.11 & 4.03 & 63.52 & $\mathrm{C}$ \\
\hline 10246. & 1.77 & 0.26 & 208. & 279.29 & 277.42 & 282.29 & 4.61 & 63.52 & $\mathrm{C}$ \\
\hline 11735. & 1.94 & 0.35 & 208. & 279.49 & 277.45 & 283.32 & 5.15 & 63.52 & $\mathrm{C}$ \\
\hline 13276. & 2.11 & 0.44 & 208. & 279.70 & 277.47 & 284.73 & 5.72 & 63.52 & $\mathrm{C}$ \\
\hline 14971. & 2.29 & 0.57 & 208. & 279.93 & 277.51 & 286.17 & 6.34 & 63.52 & $\mathrm{C}$ \\
\hline 664. & 0.30 & 0.00 & 292. & 278.25 & 277.83 & 279.14 & 0.35 & 63.52 & $\mathrm{C}$ \\
\hline 1358. & 0.50 & 0.01 & 292. & 278.44 & 277.83 & 279.18 & 0.97 & 63.52 & $\mathrm{C}$ \\
\hline 2000. & 0.69 & 0.02 & 292. & 278.62 & 277.83 & 279.35 & 1.54 & 63.52 & $\mathrm{C}$ \\
\hline 2591. & 0.86 & 0.03 & 292. & 278.78 & 277.83 & 279.70 & 2.06 & 63.52 & $\mathrm{C}$ \\
\hline 3274. & 1.06 & 0.05 & 292. & 278.97 & 277.84 & 280.03 & 2.66 & 63.52 & $\mathrm{C}$ \\
\hline 3652. & 1.16 & 0.06 & 292. & 279.07 & 277.84 & 280.34 & 3.00 & 63.52 & $\mathrm{C}$ \\
\hline 7148. & 1.42 & 0.09 & 292. & 279.28 & 277.77 & 280.64 & 3.69 & 63.52 & $\mathrm{C}$ \\
\hline 8139. & 1.52 & 0.12 & 292. & 279.39 & 277.78 & 281.37 & 4.03 & 63.52 & $\mathrm{C}$ \\
\hline 9835. & 1.70 & 0.17 & 292. & 279.57 & 277.78 & 282.51 & 4.61 & 63.52 & $\mathrm{C}$ \\
\hline 11415. & 1.88 & 0.23 & 292. & 279.73 & 277.77 & 283.55 & 5.15 & 63.52 & $\mathrm{C}$ \\
\hline 13052. & 2.07 & 0.29 & 292. & 279.91 & 277.74 & 284.99 & 5.72 & 63.52 & $\mathrm{C}$ \\
\hline 14850. & 2.28 & 0.38 & 292. & 280.10 & 277.70 & 286.47 & 6.34 & 63.52 & $\mathrm{C}$ \\
\hline 647. & 0.43 & 0.00 & 151. & 278.57 & 278.16 & 279.47 & 0.35 & 63.52 & $\mathrm{C}$ \\
\hline 1293. & 0.59 & 0.01 & 151. & 278.77 & 278.16 & 279.50 & 0.97 & 63.52 & $\mathrm{C}$ \\
\hline 1891. & 0.74 & 0.03 & 151. & 278.95 & 278.17 & 279.67 & 1.54 & 63.52 & $\mathrm{C}$ \\
\hline 2442. & 0.88 & 0.05 & 151. & 279.12 & 278.19 & 280.00 & 2.06 & 63.52 & $\mathrm{C}$ \\
\hline 3078. & 1.03 & 0.08 & 151. & 279.31 & 278.20 & 280.31 & 2.66 & 63.52 & $\mathrm{C}$ \\
\hline 3430. & 1.11 & 0.10 & 151. & 279.42 & 278.22 & 280.60 & 3.00 & 63.52 & $\mathrm{C}$ \\
\hline 6245. & 1.28 & 0.16 & 151. & 279.64 & 278.22 & 280.85 & 3.69 & 63.52 & $\mathrm{C}$ \\
\hline 6908. & 1.36 & 0.21 & 151. & 279.75 & 278.25 & 281.50 & 4.03 & 63.52 & $\mathrm{C}$ \\
\hline 8044. & 1.48 & 0.29 & 151. & 279.94 & 278.30 & 282.44 & 4.61 & 63.52 & $\mathrm{C}$ \\
\hline 9103. & 1.58 & 0.38 & 151. & 280.11 & 278.35 & 283.35 & 5.15 & 63.52 & $\mathrm{C}$ \\
\hline 10199. & 1.69 & 0.48 & 151. & 280.29 & 278.42 & 284.45 & 5.72 & 63.52 & $\mathrm{C}$ \\
\hline 11404. & 1.79 & 0.61 & 151. & 280.49 & 278.51 & 285.58 & 6.34 & 63.52 & $\mathrm{C}$ \\
\hline 424. & 0.43 & 0.00 & 104. & 278.35 & 277.93 & 279.12 & 0.35 & 63.52 & $\mathrm{C}$ \\
\hline
\end{tabular}




\begin{tabular}{|c|c|c|c|c|c|c|c|c|c|}
\hline 906. & 0.55 & 0.01 & 104. & 278.50 & 277.94 & 279.13 & 0.97 & 63.52 & $\mathrm{C}$ \\
\hline 1352. & 0.66 & 0.03 & 104. & 278.64 & 277.95 & 279.26 & 1.54 & 63.52 & $\mathrm{C}$ \\
\hline 1762. & 0.75 & 0.05 & 104. & 278.77 & 277.97 & 279.54 & 2.06 & 63.52 & $\mathrm{C}$ \\
\hline 2236. & 0.85 & 0.09 & 104. & 278.92 & 277.99 & 279.77 & 2.66 & 63.52 & $\mathrm{C}$ \\
\hline 2498. & 0.90 & 0.11 & 104. & 279.01 & 278.00 & 279.98 & 3.00 & 63.52 & $\mathrm{C}$ \\
\hline 3921. & 1.01 & 0.17 & 104. & 279.18 & 278.02 & 280.11 & 3.69 & 63.52 & $\mathrm{C}$ \\
\hline 4362. & 1.05 & 0.20 & 104. & 279.26 & 278.05 & 280.60 & 4.03 & 63.52 & $\mathrm{C}$ \\
\hline 5119. & 1.12 & 0.28 & 104. & 279.41 & 278.11 & 281.14 & 4.61 & 63.52 & $\mathrm{C}$ \\
\hline 5825. & 1.17 & 0.36 & 104. & 279.54 & 278.17 & 281.76 & 5.15 & 63.52 & $\mathrm{C}$ \\
\hline 6556. & 1.22 & 0.46 & 104. & 279.68 & 278.24 & 282.47 & 5.72 & 63.52 & $\mathrm{C}$ \\
\hline 7360. & 1.26 & 0.58 & 104. & 279.84 & 278.34 & 283.21 & 6.34 & 63.52 & $\mathrm{C}$ \\
\hline 19679. & 2.99 & 0.09 & 222. & 280.96 & 278.05 & 286.93 & 0.35 & 63.52 & $\mathrm{P}$ \\
\hline 16147. & 2.58 & 0.23 & 222. & 280.66 & 278.14 & 284.97 & 0.97 & 63.52 & $\mathrm{P}$ \\
\hline 12874. & 2.22 & 0.34 & 222. & 280.37 & 278.20 & 283.64 & 1.54 & 63.52 & $\mathrm{P}$ \\
\hline 9857. & 1.91 & 0.41 & 222. & 280.11 & 278.24 & 282.48 & 2.06 & 63.52 & $\mathrm{P}$ \\
\hline 6363. & 1.58 & 0.48 & 222. & 279.81 & 278.26 & 281.63 & 2.66 & 63.52 & $\mathrm{P}$ \\
\hline 4427. & 1.40 & 0.50 & 222. & 279.64 & 278.26 & 281.08 & 3.00 & 63.52 & $\mathrm{P}$ \\
\hline 6850. & 1.18 & 0.55 & 222. & 279.29 & 278.01 & 280.59 & 3.69 & 63.52 & $\mathrm{P}$ \\
\hline 5983. & 1.01 & 0.58 & 222. & 279.12 & 278.00 & 280.22 & 4.03 & 63.52 & $\mathrm{P}$ \\
\hline 4496. & 0.75 & 0.62 & 222. & 278.83 & 277.98 & 279.55 & 4.61 & 63.52 & $\mathrm{P}$ \\
\hline 3108. & 0.52 & 0.65 & 222. & 278.56 & 277.94 & 279.30 & 5.15 & 63.52 & $\mathrm{P}$ \\
\hline 1668. & 0.30 & 0.67 & 222. & 278.28 & 277.88 & 279.00 & 5.72 & 63.52 & $\mathrm{P}$ \\
\hline 82. & 0.08 & 0.68 & 222. & 277.97 & 277.78 & 278.98 & 6.34 & 63.52 & $\mathrm{P}$ \\
\hline 19409. & 2.95 & 0.07 & 291. & 281.21 & 278.29 & 287.08 & 0.35 & 63.52 & $\mathrm{P}$ \\
\hline 15943. & 2.57 & 0.17 & 291. & 280.88 & 278.34 & 285.13 & 0.97 & 63.52 & $\mathrm{P}$ \\
\hline 12733. & 2.23 & 0.25 & 291. & 280.57 & 278.37 & 283.80 & 1.54 & 63.52 & $\mathrm{P}$ \\
\hline 9774. & 1.94 & 0.31 & 291. & 280.28 & 278.38 & 282.65 & 2.06 & 63.52 & $\mathrm{P}$ \\
\hline 6346. & 1.62 & 0.36 & 291. & 279.95 & 278.36 & 281.80 & 2.66 & 63.52 & $\mathrm{P}$ \\
\hline 4447. & 1.46 & 0.38 & 291. & 279.77 & 278.34 & 281.25 & 3.00 & 63.52 & $\mathrm{P}$ \\
\hline 7198. & 1.28 & 0.42 & 291. & 279.39 & 277.99 & 280.73 & 3.69 & 63.52 & $\mathrm{P}$ \\
\hline 6291. & 1.12 & 0.44 & 291. & 279.20 & 277.97 & 280.35 & 4.03 & 63.52 & $\mathrm{P}$ \\
\hline 4734. & 0.87 & 0.47 & 291. & 278.89 & 277.91 & 279.64 & 4.61 & 63.52 & $\mathrm{P}$ \\
\hline 3280. & 0.65 & 0.49 & 291. & 278.59 & 277.84 & 279.36 & 5.15 & 63.52 & $\mathrm{P}$ \\
\hline 1772. & 0.44 & 0.51 & 291. & 278.28 & 277.75 & 279.04 & 5.72 & 63.52 & $\mathrm{P}$ \\
\hline 11220. & 0.23 & 0.52 & 291. & 277.94 & 277.62 & 279.01 & 6.34 & 63.52 & $\mathrm{P}$ \\
\hline 15712. & 2.57 & 0.10 & 163. & 281.03 & 278.51 & 286.00 & 0.35 & 63.52 & $\mathrm{P}$ \\
\hline 12842. & 2.21 & 0.25 & 163. & 280.80 & 278.62 & 284.36 & 0.97 & 63.52 & $\mathrm{P}$ \\
\hline 10183. & 1.90 & 0.37 & 163. & 280.58 & 278.71 & 283.27 & 1.54 & 63.52 & $\mathrm{P}$ \\
\hline 7731. & 1.63 & 0.45 & 163. & 280.38 & 278.78 & 282.34 & 2.06 & 63.52 & $\mathrm{P}$ \\
\hline 4893. & 1.33 & 0.52 & 163. & 280.16 & 278.83 & 281.64 & 2.66 & 63.52 & $\mathrm{P}$ \\
\hline 3320. & 1.18 & 0.55 & 163. & 280.03 & 278.84 & 281.20 & 3.00 & 63.52 & $\mathrm{P}$ \\
\hline 5285. & 0.95 & 0.60 & 163. & 279.77 & 278.72 & 280.80 & 3.69 & 63.52 & $\mathrm{P}$ \\
\hline 4572. & 0.80 & 0.63 & 163. & 279.64 & 278.74 & 280.52 & 4.03 & 63.52 & $\mathrm{P}$ \\
\hline 3349. & 0.57 & 0.67 & 163. & 279.42 & 278.75 & 279.99 & 4.61 & 63.52 & $\mathrm{P}$ \\
\hline 2206. & 0.37 & 0.70 & 163. & 279.22 & 278.75 & 279.80 & 5.15 & 63.52 & $\mathrm{P}$ \\
\hline
\end{tabular}




\begin{tabular}{|c|c|c|c|c|c|c|c|c|c|}
\hline 1021. & 0.18 & 0.72 & 163. & 279.00 & 278.72 & 279.58 & 5.72 & 63.52 & $\mathrm{P}$ \\
\hline 12397. & 1.98 & 0.13 & 100. & 280.10 & 278.10 & 283.79 & 0.35 & 63.52 & $\mathrm{P}$ \\
\hline 10132. & 1.68 & 0.32 & 100. & 279.95 & 278.26 & 282.62 & 0.97 & 63.52 & $\mathrm{P}$ \\
\hline 8034. & 1.41 & 0.47 & 100. & 279.81 & 278.41 & 281.87 & 1.54 & 63.52 & $\mathrm{P}$ \\
\hline 6100. & 1.18 & 0.58 & 100. & 279.68 & 278.53 & 281.25 & 2.06 & 63.52 & $\mathrm{P}$ \\
\hline 3862. & 0.93 & 0.67 & 100. & 279.53 & 278.64 & 280.75 & 2.66 & 63.52 & $\mathrm{P}$ \\
\hline 2622. & 0.80 & 0.70 & 100. & 279.44 & 278.69 & 280.43 & 3.00 & 63.52 & $\mathrm{P}$ \\
\hline 4421. & 0.58 & 0.77 & 100. & 279.27 & 278.70 & 280.12 & 3.69 & 63.52 & $\mathrm{P}$ \\
\hline 3784. & 0.45 & 0.82 & 100. & 279.19 & 278.76 & 279.95 & 4.03 & 63.52 & $\mathrm{P}$ \\
\hline 2691. & 0.24 & 0.88 & 100. & 279.04 & 278.85 & 279.52 & 4.61 & 63.52 & $\mathrm{P}$ \\
\hline 1671. & 0.07 & 0.91 & 100. & 278.91 & 278.89 & 279.40 & 5.15 & 63.52 & $\mathrm{P}$ \\
\hline 612. & -.08 & 0.93 & 100. & 278.77 & 278.89 & 279.26 & 5.72 & 63.52 & $\mathrm{P}$ \\
\hline 865. & 0.43 & 0.00 & 220. & 278.27 & 277.86 & 279.21 & 0.35 & 63.52 & $\mathrm{C}$ \\
\hline 1670. & 0.61 & 0.01 & 220. & 278.49 & 277.86 & 279.27 & 0.97 & 63.52 & $\mathrm{C}$ \\
\hline 2415. & 0.78 & 0.03 & 220. & 278.68 & 277.87 & 279.47 & 1.54 & 63.52 & $\mathrm{C}$ \\
\hline 3100. & 0.94 & 0.05 & 220. & 278.86 & 277.88 & 279.83 & 2.06 & 63.52 & $\mathrm{C}$ \\
\hline 3892. & 1.12 & 0.07 & 220. & 279.07 & 277.89 & 280.18 & 2.66 & 63.52 & $\mathrm{C}$ \\
\hline 4331. & 1.21 & 0.09 & 220. & 279.19 & 277.90 & 280.55 & 3.00 & 63.52 & $\mathrm{C}$ \\
\hline 7678. & 1.43 & 0.14 & 220. & 279.42 & 277.89 & 280.84 & 3.69 & 63.52 & $\mathrm{C}$ \\
\hline 8723. & 1.53 & 0.18 & 220. & 279.54 & 277.91 & 281.61 & 4.03 & 63.52 & $\mathrm{C}$ \\
\hline 10513. & 1.69 & 0.25 & 220. & 279.74 & 277.94 & 282.77 & 4.61 & 63.52 & $\mathrm{C}$ \\
\hline 12181. & 1.85 & 0.33 & 220. & 279.93 & 277.96 & 283.84 & 5.15 & 63.52 & $\mathrm{C}$ \\
\hline 13908. & 2.01 & 0.43 & 220. & 280.12 & 277.99 & 285.29 & 5.72 & 63.52 & $\mathrm{C}$ \\
\hline 15807. & 2.19 & 0.55 & 220. & 280.34 & 278.01 & 286.80 & 6.34 & 63.52 & $\mathrm{C}$ \\
\hline 811. & 0.45 & 0.00 & 300. & 278.27 & 277.87 & 279.16 & 0.35 & 63.52 & $\mathrm{C}$ \\
\hline 1544. & 0.61 & 0.01 & 300. & 278.46 & 277.86 & 279.22 & 0.97 & 63.52 & $\mathrm{C}$ \\
\hline 2222. & 0.76 & 0.02 & 300. & 278.64 & 277.86 & 279.40 & 1.54 & 63.52 & $\mathrm{C}$ \\
\hline 2846. & 0.91 & 0.03 & 300. & 278.80 & 277.87 & 279.75 & 2.06 & 63.52 & $\mathrm{C}$ \\
\hline 3567. & 1.08 & 0.05 & 300. & 278.99 & 277.87 & 280.08 & 2.66 & 63.52 & $\mathrm{C}$ \\
\hline 3965. & 1.17 & 0.06 & 300. & 279.10 & 277.87 & 280.43 & 3.00 & 63.52 & $\mathrm{C}$ \\
\hline 7188. & 1.41 & 0.10 & 300. & 279.31 & 277.81 & 280.71 & 3.69 & 63.52 & $\mathrm{C}$ \\
\hline 8288. & 1.51 & 0.12 & 300. & 279.42 & 277.82 & 281.45 & 4.03 & 63.52 & $\mathrm{C}$ \\
\hline 10172. & 1.69 & 0.17 & 300. & 279.60 & 277.81 & 282.58 & 4.61 & 63.52 & $\mathrm{C}$ \\
\hline 11927. & 1.86 & 0.23 & 300. & 279.77 & 277.80 & 283.64 & 5.15 & 63.52 & $\mathrm{C}$ \\
\hline 13745. & 2.06 & 0.30 & 300. & 279.94 & 277.78 & 285.10 & 5.72 & 63.52 & $\mathrm{C}$ \\
\hline 15743. & 2.28 & 0.38 & 300. & 280.14 & 277.74 & 286.63 & 6.34 & 63.52 & $\mathrm{C}$ \\
\hline 428. & 0.41 & 0.00 & 157. & 278.37 & 277.97 & 279.33 & 0.35 & 63.52 & $\mathrm{C}$ \\
\hline 1242. & 0.59 & 0.01 & 157. & 278.58 & 277.98 & 279.34 & 0.97 & 63.52 & $\mathrm{C}$ \\
\hline 1995. & 0.76 & 0.03 & 157. & 278.78 & 277.99 & 279.53 & 1.54 & 63.52 & $\mathrm{C}$ \\
\hline 2688. & 0.91 & 0.05 & 157. & 278.96 & 278.00 & 279.86 & 2.06 & 63.52 & $\mathrm{C}$ \\
\hline 3488. & 1.07 & 0.08 & 157. & 279.17 & 278.02 & 280.18 & 2.66 & 63.52 & $\mathrm{C}$ \\
\hline 3932. & 1.16 & 0.10 & 157. & 279.28 & 278.04 & 280.52 & 3.00 & 63.52 & $\mathrm{C}$ \\
\hline 6197. & 1.35 & 0.17 & 157. & 279.52 & 278.04 & 280.78 & 3.69 & 63.52 & $\mathrm{C}$ \\
\hline 6971. & 1.43 & 0.21 & 157. & 279.64 & 278.07 & 281.44 & 4.03 & 63.52 & $\mathrm{C}$ \\
\hline 8297. & 1.56 & 0.29 & 157. & 279.84 & 278.12 & 282.38 & 4.61 & 63.52 & $\mathrm{C}$ \\
\hline
\end{tabular}




\begin{tabular}{|c|c|c|c|c|c|c|c|c|c|}
\hline 9533. & 1.68 & 0.38 & 157. & 280.02 & 278.17 & 283.24 & 5.15 & 63.52 & $\mathrm{C}$ \\
\hline 10813. & 1.80 & 0.48 & 157. & 280.22 & 278.23 & 284.41 & 5.72 & 63.52 & $\mathrm{C}$ \\
\hline 12220. & 1.91 & 0.62 & 157. & 280.43 & 278.33 & 285.63 & 6.34 & 63.52 & $\mathrm{C}$ \\
\hline 205. & 0.41 & 0.00 & 99. & 278.71 & 278.31 & 279.54 & 0.35 & 63.52 & $\mathrm{C}$ \\
\hline 819. & 0.55 & 0.01 & 99. & 278.88 & 278.31 & 279.53 & 0.97 & 63.52 & $\mathrm{C}$ \\
\hline 1387. & 0.67 & 0.03 & 99. & 279.04 & 278.32 & 279.67 & 1.54 & 63.52 & $\mathrm{C}$ \\
\hline 1910. & 0.78 & 0.05 & 99. & 279.19 & 278.34 & 279.93 & 2.06 & 63.52 & $\mathrm{C}$ \\
\hline 2514. & 0.90 & 0.09 & 99. & 279.36 & 278.37 & 280.17 & 2.66 & 63.52 & $\mathrm{C}$ \\
\hline 2849. & 0.96 & 0.11 & 99. & 279.46 & 278.38 & 280.41 & 3.00 & 63.52 & $\mathrm{C}$ \\
\hline 3870. & 1.09 & 0.18 & 99. & 279.65 & 278.40 & 280.55 & 3.69 & 63.52 & $\mathrm{C}$ \\
\hline 4333. & 1.15 & 0.22 & 99. & 279.75 & 278.44 & 281.03 & 4.03 & 63.52 & $\mathrm{C}$ \\
\hline 5125. & 1.23 & 0.30 & 99. & 279.91 & 278.50 & 281.58 & 4.61 & 63.52 & $\mathrm{C}$ \\
\hline 5864. & 1.30 & 0.39 & 99. & 280.07 & 278.57 & 282.14 & 5.15 & 63.52 & $\mathrm{C}$ \\
\hline 6629. & 1.37 & 0.49 & 99. & 280.23 & 278.65 & 282.88 & 5.72 & 63.52 & $\mathrm{C}$ \\
\hline 7470. & 1.42 & 0.62 & 99. & 280.40 & 278.78 & 283.64 & 6.34 & 63.52 & $\mathrm{C}$ \\
\hline 189. & 0.50 & 0.00 & 100. & 278.86 & 278.36 & 279.70 & 0.35 & 63.52 & $\mathrm{C}$ \\
\hline 809. & 0.58 & 0.01 & 100. & 279.03 & 278.48 & 279.69 & 0.97 & 63.52 & $\mathrm{C}$ \\
\hline 1383. & 0.69 & 0.03 & 100. & 279.19 & 278.49 & 279.82 & 1.54 & 63.52 & $\mathrm{C}$ \\
\hline 1910. & 0.79 & 0.05 & 100. & 279.34 & 278.50 & 280.09 & 2.06 & 63.52 & $\mathrm{C}$ \\
\hline 2520. & 0.90 & 0.09 & 100. & 279.51 & 278.53 & 280.32 & 2.66 & 63.52 & $\mathrm{C}$ \\
\hline 2858. & 0.96 & 0.11 & 100. & 279.61 & 278.55 & 280.57 & 3.00 & 63.52 & $\mathrm{C}$ \\
\hline 3836. & 1.08 & 0.18 & 100. & 279.80 & 278.57 & 280.69 & 3.69 & 63.52 & $\mathrm{C}$ \\
\hline 4299. & 1.13 & 0.22 & 100. & 279.90 & 278.61 & 281.17 & 4.03 & 63.52 & $\mathrm{C}$ \\
\hline 5092. & 1.21 & 0.30 & 100. & 280.07 & 278.67 & 281.72 & 4.61 & 63.52 & $\mathrm{C}$ \\
\hline 5831. & 1.29 & 0.38 & 100. & 280.22 & 278.74 & 282.28 & 5.15 & 63.52 & $\mathrm{C}$ \\
\hline 6596. & 1.35 & 0.48 & 100. & 280.38 & 278.82 & 283.02 & 5.72 & 63.52 & $\mathrm{C}$ \\
\hline 7439. & 1.41 & 0.61 & 100. & 280.56 & 278.94 & 283.77 & 6.34 & 63.52 & $\mathrm{C}$ \\
\hline 20314. & 2.94 & 0.10 & 219. & 280.75 & 277.80 & 286.97 & 0.35 & 63.52 & $\mathrm{P}$ \\
\hline 16667. & 2.55 & 0.24 & 219. & 280.43 & 277.90 & 284.89 & 0.97 & 63.52 & $\mathrm{P}$ \\
\hline 13288. & 2.20 & 0.35 & 219. & 280.15 & 277.97 & 283.53 & 1.54 & 63.52 & $\mathrm{P}$ \\
\hline 10173. & 1.89 & 0.44 & 219. & 279.88 & 278.01 & 282.35 & 2.06 & 63.52 & $\mathrm{P}$ \\
\hline 6565. & 1.56 & 0.50 & 219. & 279.57 & 278.03 & 281.47 & 2.66 & 63.52 & $\mathrm{P}$ \\
\hline 4566. & 1.39 & 0.53 & 219. & 279.40 & 278.03 & 280.88 & 3.00 & 63.52 & $\mathrm{P}$ \\
\hline 6796. & 1.17 & 0.58 & 219. & 279.05 & 277.78 & 280.39 & 3.69 & 63.52 & $\mathrm{P}$ \\
\hline 5944. & 1.01 & 0.61 & 219. & 278.88 & 277.78 & 280.01 & 4.03 & 63.52 & $\mathrm{P}$ \\
\hline 4484. & 0.74 & 0.65 & 219. & 278.58 & 277.76 & 279.33 & 4.61 & 63.52 & $\mathrm{P}$ \\
\hline 3120. & 0.50 & 0.68 & 219. & 278.31 & 277.72 & 279.07 & 5.15 & 63.52 & $\mathrm{P}$ \\
\hline 1704. & 0.27 & 0.70 & 219. & 278.02 & 277.66 & 278.77 & 5.72 & 63.52 & $\mathrm{P}$ \\
\hline 145. & 0.05 & 0.71 & 219. & 277.71 & 277.57 & 278.74 & 6.34 & 63.52 & $\mathrm{P}$ \\
\hline 19520. & 2.94 & 0.07 & 294. & 281.06 & 278.14 & 286.89 & 0.35 & 63.52 & $\mathrm{P}$ \\
\hline 16016. & 2.56 & 0.17 & 294. & 280.73 & 278.19 & 284.92 & 0.97 & 63.52 & $\mathrm{P}$ \\
\hline 12770. & 2.23 & 0.25 & 294. & 280.42 & 278.22 & 283.63 & 1.54 & 63.52 & $\mathrm{P}$ \\
\hline 9777. & 1.94 & 0.31 & 294. & 280.13 & 278.23 & 282.49 & 2.06 & 63.52 & $\mathrm{P}$ \\
\hline 6311. & 1.63 & 0.36 & 294. & 279.80 & 278.21 & 281.65 & 2.66 & 63.52 & $\mathrm{P}$ \\
\hline 4390. & 1.46 & 0.38 & 294. & 279.62 & 278.19 & 281.10 & 3.00 & 63.52 & $\mathrm{P}$ \\
\hline
\end{tabular}




\begin{tabular}{|c|c|c|c|c|c|c|c|c|c|}
\hline 6987. & 1.29 & 0.41 & 294. & 279.25 & 277.84 & 280.59 & 3.69 & 63.52 & $\mathrm{P}$ \\
\hline 6140. & 1.14 & 0.44 & 294. & 279.06 & 277.81 & 280.20 & 4.03 & 63.52 & $\mathrm{P}$ \\
\hline 4685. & 0.89 & 0.47 & 294. & 278.74 & 277.75 & 279.51 & 4.61 & 63.52 & $\mathrm{P}$ \\
\hline 3327. & 0.67 & 0.49 & 294. & 278.45 & 277.68 & 279.24 & 5.15 & 63.52 & $\mathrm{P}$ \\
\hline 1918. & 0.47 & 0.50 & 294. & 278.14 & 277.58 & 278.92 & 5.72 & 63.52 & $\mathrm{P}$ \\
\hline 365. & 0.27 & 0.51 & 294. & 277.80 & 277.45 & 278.87 & 6.34 & 63.52 & $\mathrm{P}$ \\
\hline 17093. & 2.60 & 0.11 & 162. & 281.29 & 278.67 & 286.58 & 0.35 & 63.52 & $\mathrm{P}$ \\
\hline 13985. & 2.24 & 0.28 & 162. & 281.05 & 278.80 & 284.84 & 0.97 & 63.52 & $\mathrm{P}$ \\
\hline 11107. & 1.93 & 0.40 & 162. & 280.83 & 278.90 & 283.70 & 1.54 & 63.52 & $\mathrm{P}$ \\
\hline 8453. & 1.65 & 0.50 & 162. & 280.63 & 278.98 & 282.71 & 2.06 & 63.52 & $\mathrm{P}$ \\
\hline 5381. & 1.36 & 0.57 & 162. & 280.40 & 279.04 & 282.01 & 2.66 & 63.52 & $\mathrm{P}$ \\
\hline 3678. & 1.21 & 0.60 & 162. & 280.27 & 279.06 & 281.53 & 3.00 & 63.52 & $\mathrm{P}$ \\
\hline 5593. & 0.98 & 0.66 & 162. & 280.01 & 278.94 & 281.13 & 3.69 & 63.52 & $\mathrm{P}$ \\
\hline 4854. & 0.83 & 0.69 & 162. & 279.88 & 278.97 & 280.83 & 4.03 & 63.52 & $\mathrm{P}$ \\
\hline 3585. & 0.59 & 0.74 & 162. & 279.65 & 278.99 & 280.27 & 4.61 & 63.52 & $\mathrm{P}$ \\
\hline 2401. & 0.38 & 0.77 & 162. & 279.44 & 278.99 & 280.06 & 5.15 & 63.52 & $\mathrm{P}$ \\
\hline 1172. & 0.18 & 0.79 & 162. & 279.23 & 278.97 & 279.84 & 5.72 & 63.52 & $\mathrm{P}$ \\
\hline 11120. & 2.03 & 0.11 & 104. & 280.56 & 278.51 & 284.16 & 0.35 & 63.52 & $\mathrm{P}$ \\
\hline 9056. & 1.73 & 0.28 & 104. & 280.41 & 278.65 & 282.99 & 0.97 & 63.52 & $\mathrm{P}$ \\
\hline 7145. & 1.47 & 0.41 & 104. & 280.26 & 278.77 & 282.24 & 1.54 & 63.52 & $\mathrm{P}$ \\
\hline 5384. & 1.25 & 0.50 & 104. & 280.13 & 278.87 & 281.62 & 2.06 & 63.52 & $\mathrm{P}$ \\
\hline 3345. & 1.01 & 0.58 & 104. & 279.98 & 278.95 & 281.13 & 2.66 & 63.52 & $\mathrm{P}$ \\
\hline 2215. & 0.89 & 0.60 & 104. & 279.90 & 278.98 & 280.82 & 3.00 & 63.52 & $\mathrm{P}$ \\
\hline 3886. & 0.68 & 0.66 & 104. & 279.72 & 278.97 & 280.51 & 3.69 & 63.52 & $\mathrm{P}$ \\
\hline 3305. & 0.56 & 0.69 & 104. & 279.64 & 279.01 & 280.35 & 4.03 & 63.52 & $\mathrm{P}$ \\
\hline 2308. & 0.36 & 0.74 & 104. & 279.49 & 279.07 & 279.93 & 4.61 & 63.52 & $\mathrm{P}$ \\
\hline 1378. & 0.20 & 0.77 & 104. & 279.36 & 279.09 & 279.82 & 5.15 & 63.52 & $\mathrm{P}$ \\
\hline 412. & 0.05 & 0.79 & 104. & 279.21 & 279.08 & 279.68 & 5.72 & 63.52 & $\mathrm{P}$ \\
\hline 11347. & 2.06 & 0.12 & 102. & 280.42 & 278.35 & 284.01 & 0.35 & 63.52 & $\mathrm{P}$ \\
\hline 9252. & 1.76 & 0.29 & 102. & 280.27 & 278.49 & 282.83 & 0.97 & 63.52 & $\mathrm{P}$ \\
\hline 7312. & 1.49 & 0.43 & 102. & 280.13 & 278.62 & 282.11 & 1.54 & 63.52 & $\mathrm{P}$ \\
\hline 5524. & 1.25 & 0.52 & 102. & 279.99 & 278.73 & 281.49 & 2.06 & 63.52 & $\mathrm{P}$ \\
\hline 3454. & 1.00 & 0.60 & 102. & 279.84 & 278.82 & 281.01 & 2.66 & 63.52 & $\mathrm{P}$ \\
\hline 2308. & 0.88 & 0.63 & 102. & 279.76 & 278.85 & 280.69 & 3.00 & 63.52 & $\mathrm{P}$ \\
\hline 3887. & 0.66 & 0.69 & 102. & 279.58 & 278.85 & 280.38 & 3.69 & 63.52 & $\mathrm{P}$ \\
\hline 3325. & 0.53 & 0.73 & 102. & 279.50 & 278.90 & 280.23 & 4.03 & 63.52 & $\mathrm{P}$ \\
\hline 2362. & 0.33 & 0.78 & 102. & 279.35 & 278.96 & 279.81 & 4.61 & 63.52 & $\mathrm{P}$ \\
\hline 1463. & 0.16 & 0.81 & 102. & 279.22 & 278.99 & 279.70 & 5.15 & 63.52 & $\mathrm{P}$ \\
\hline 530. & 0.01 & 0.82 & 102. & 279.07 & 278.98 & 279.56 & 5.72 & 63.52 & $\mathrm{P}$ \\
\hline 523. & 0.65 & 0.00 & 103. & 278.97 & 278.31 & 279.52 & 0.97 & 63.52 & $\mathrm{C}$ \\
\hline 1183. & 0.74 & 0.02 & 103. & 279.10 & 278.31 & 279.64 & 1.54 & 63.52 & $\mathrm{C}$ \\
\hline 1790. & 0.81 & 0.04 & 103. & 279.22 & 278.33 & 279.89 & 2.06 & 63.52 & $\mathrm{C}$ \\
\hline 2492. & 0.90 & 0.07 & 103. & 279.36 & 278.35 & 280.12 & 2.66 & 63.52 & $\mathrm{C}$ \\
\hline 2880. & 0.94 & 0.10 & 103. & 279.44 & 278.37 & 280.36 & 3.00 & 63.52 & $\mathrm{C}$ \\
\hline 3795. & 1.05 & 0.16 & 103. & 279.60 & 278.38 & 280.47 & 3.69 & 63.52 & $\mathrm{C}$ \\
\hline
\end{tabular}




\begin{tabular}{|c|c|c|c|c|c|c|c|c|c|}
\hline 4202. & 1.08 & 0.20 & 103. & 279.68 & 278.42 & 280.92 & 4.03 & 63.52 & $\mathrm{C}$ \\
\hline 4899. & 1.15 & 0.27 & 103. & 279.81 & 278.47 & 281.48 & 4.61 & 63.52 & $\mathrm{C}$ \\
\hline 5549. & 1.20 & 0.35 & 103. & 279.94 & 278.53 & 282.02 & 5.15 & 63.52 & $\mathrm{C}$ \\
\hline 6223. & 1.26 & 0.44 & 103. & 280.07 & 278.60 & 282.71 & 5.72 & 63.52 & $\mathrm{C}$ \\
\hline 6963. & 1.31 & 0.56 & 103. & 280.21 & 278.70 & 283.39 & 6.34 & 63.52 & $\mathrm{C}$ \\
\hline 10232. & 2.06 & 0.11 & 99. & 280.32 & 278.25 & 283.64 & 0.35 & 63.52 & $\mathrm{P}$ \\
\hline 8312. & 1.76 & 0.27 & 99. & 280.17 & 278.38 & 282.52 & 0.97 & 63.52 & $\mathrm{P}$ \\
\hline 6533. & 1.49 & 0.39 & 99. & 280.02 & 278.49 & 281.85 & 1.54 & 63.52 & $\mathrm{P}$ \\
\hline 4894. & 1.26 & 0.48 & 99. & 279.89 & 278.58 & 281.32 & 2.06 & 63.52 & $\mathrm{P}$ \\
\hline 2997. & 1.02 & 0.55 & 99. & 279.74 & 278.66 & 280.88 & 2.66 & 63.52 & $\mathrm{P}$ \\
\hline 1945. & 0.90 & 0.57 & 99. & 279.65 & 278.69 & 280.58 & 3.00 & 63.52 & $\mathrm{P}$ \\
\hline 3714. & 0.69 & 0.63 & 99. & 279.48 & 278.69 & 280.28 & 3.69 & 63.52 & $\mathrm{P}$ \\
\hline 3168. & 0.56 & 0.67 & 99. & 279.39 & 278.73 & 280.12 & 4.03 & 63.52 & $\mathrm{P}$ \\
\hline 2232. & 0.37 & 0.71 & 99. & 279.25 & 278.79 & 279.71 & 4.61 & 63.52 & $\mathrm{P}$ \\
\hline 1358. & 0.20 & 0.74 & 99. & 279.11 & 278.81 & 279.60 & 5.15 & 63.52 & $\mathrm{P}$ \\
\hline 452. & 0.06 & 0.76 & 99. & 278.97 & 278.80 & 279.47 & 5.72 & 63.52 & $\mathrm{P}$ \\
\hline 666. & 0.64 & 0.01 & 107. & 279.02 & 278.35 & 279.63 & 0.97 & 63.52 & $\mathrm{C}$ \\
\hline 1280. & 0.73 & 0.02 & 107. & 279.16 & 278.36 & 279.74 & 1.54 & 63.52 & $\mathrm{C}$ \\
\hline 1845. & 0.82 & 0.04 & 107. & 279.29 & 278.37 & 280.01 & 2.06 & 63.52 & $\mathrm{C}$ \\
\hline 2498. & 0.91 & 0.08 & 107. & 279.43 & 278.40 & 280.24 & 2.66 & 63.52 & $\mathrm{C}$ \\
\hline 2859. & 0.96 & 0.10 & 107. & 279.51 & 278.41 & 280.47 & 3.00 & 63.52 & $\mathrm{C}$ \\
\hline 3836. & 1.08 & 0.16 & 107. & 279.68 & 278.43 & 280.58 & 3.69 & 63.52 & $\mathrm{C}$ \\
\hline 4263. & 1.12 & 0.20 & 107. & 279.76 & 278.46 & 281.03 & 4.03 & 63.52 & $\mathrm{C}$ \\
\hline 4994. & 1.20 & 0.27 & 107. & 279.90 & 278.52 & 281.57 & 4.61 & 63.52 & $\mathrm{C}$ \\
\hline 5675. & 1.26 & 0.35 & 107. & 280.04 & 278.58 & 282.11 & 5.15 & 63.52 & $\mathrm{C}$ \\
\hline 6381. & 1.33 & 0.44 & 107. & 280.17 & 278.64 & 282.80 & 5.72 & 63.52 & $\mathrm{C}$ \\
\hline 7158. & 1.39 & 0.55 & 107. & 280.32 & 278.73 & 283.48 & 6.34 & 63.52 & $\mathrm{C}$ \\
\hline
\end{tabular}

